\title{
INFLUÊNCIA DO EFEITO KONDO NA CONDUTÂNCIA DE CONTATOS PONTUAIS DE SUPERFÍCIES METÁLICAS
}

\author{
Antonio Carlos Ferreira Seridonio
}

Dissertação apresentada ao Instituto de Física de São Carlos, Universidade de São Paulo, para obtenção do Título de Mestre em Física Básica.

Orientador: Prof. Dr. Luiz Nunes de Oliveira

São Carlos

2002 


$$
\begin{array}{r}
N_{+1}\left\langle 2, r_{2}, S, Q\left|H_{N I}\right| Q, S, r_{4}, 4\right\rangle_{N+1}=\left(\frac{2 S}{2 S+1}\right)^{1 / 2} \times \\
\times_{N}\left\langle r_{2}, S-1 / 2, Q-1\left\|f_{N}^{+}\right\| Q-2, S, r_{4}\right\rangle_{N},
\end{array}
$$

$$
\begin{aligned}
& N_{+1}\left\langle 3, r_{3}, S,\right.\left.Q\left|H_{N I}\right| Q, S, r_{4}, 4\right\rangle_{N+1}=-\left(\frac{2 S+2}{2 S+1}\right)^{1 / 2} \times \\
& \times_{N}\left\langle r_{3}, S+1 / 2, Q-1\left\|f_{N}^{+}\right\| Q-2, S, r_{4}\right\rangle_{N} .
\end{aligned}
$$

Percebemos que ao conhecer os autovalores $E_{N}(Q . S, r)$ e os elementos $\left\langle\left|H_{N I}\right|\right\rangle$, podemos construir a matriz $H_{N+1}$ para diagonalizá-la, determinando seus autovetores e suas autoenergias. Devemos lembrar que a matriz $H_{N+1}$ possui subespaços $(Q, S)$. Para um dado subespaço (Q,S) (bloco dentro da matriz $\left.H_{N+1}\right)$, podemos escrever seus autoestados como sendo

$$
|Q, S, \omega\rangle_{N+1}=\sum_{r, i} U_{Q S}(\omega ; r i)|Q, S, r, i\rangle_{N+1}
$$

e os autovalores correspondentes são os elementos $E_{N+1}(Q, S, \omega)$. O índice $\omega$ diferencia autoestados de um mesmo subespaço $(Q, S)$.

O passo seguinte, a iteração $N+2$, além de se utilizar dos resultados $|Q, S, \omega\rangle_{N+1}$ e $E_{N+1}(Q, S, \omega)$, também precisa dos elementos $\left\langle\left\|f_{N+1}^{+}\right\|\right\rangle$.

Combinando os resultados anteriores, obtemos

$$
\left\langle\omega, S, Q\left\|f_{N+1}^{+}\right\| Q^{\prime}, S^{\prime}, \omega^{\prime}\right\rangle=\sum_{r} U_{Q S}(\omega ; r k) U_{Q, S},\left(\omega^{\prime} ; r 1\right)+
$$




$$
\begin{array}{r}
e^{i \mu_{P} N_{P} \tau / \hbar} H_{t} e^{-i \mu_{P} N_{P} \tau / \hbar}= \\
t \sum_{k \sigma q \delta} e^{-i \vec{k} \cdot \overrightarrow{R_{2}}} c_{s k \sigma}^{+} c_{p q \delta}\left(1-\frac{i}{\hbar} \mu_{P} \tau+\frac{1}{2 !}\left(\frac{i}{\hbar} \mu_{P} \tau\right)^{2}+\cdots\right)+ \\
+t \sum_{k \sigma q \delta} e^{i \vec{k} \cdot \overrightarrow{R_{2}}} c_{p q \delta}^{+} c_{s k \sigma}\left(1+\frac{i}{\hbar} \mu_{P} \tau+\frac{1}{2 !}\left(\frac{i}{\hbar} \mu_{P} \tau\right)^{2}+\cdots\right)
\end{array}
$$

$$
=t \sum_{k \sigma} \sum_{q \delta}\left(e^{-i \vec{k} \cdot \overrightarrow{R_{2}}} c_{s k \sigma}^{+} c_{p q \delta} e^{-\frac{i}{\hbar} \mu_{P} \tau}+\text { h.c. }\right) .
$$

A expansão do comutador que aparece na expressão (3.5) precisou dos passos seguintes. Começemos pela definição de média termodinâmica,

$$
\langle O\rangle=Z^{-1} \sum_{n} e^{-\beta E_{n}}\langle n|O| n\rangle .
$$

Fazendo a aplicação dessa definição,

$$
\begin{gathered}
\left\langle\left[c_{p k_{1}}^{+}(\tau) c_{s k_{2}}(\tau), c_{s k_{3}}^{+}\left(\tau^{\prime}\right) c_{p k_{4}}\left(\tau^{\prime}\right)\right]\right\rangle=Z^{-1} \sum_{n}\left\langle n\left|\left[c_{p k_{1}}^{+}(\tau) c_{s k_{2}}(\tau), c_{s k_{3}}^{+}\left(\tau^{\prime}\right) c_{p k_{4}}\left(\tau^{\prime}\right)\right]\right| n\right\rangle e^{-\beta E} \\
=Z^{-1} \sum_{n}\left\langle n\left|c_{p k_{1}}^{+}(\tau) c_{s k_{2}}(\tau) c_{s k_{3}}^{+}\left(\tau^{\prime}\right) c_{p k_{4}}\left(\tau^{\prime}\right)\right| n\right\rangle e^{-\beta E_{n}}+ \\
-Z^{-1} \sum_{n}\left\langle n\left|c_{s k_{3}}^{+}\left(\tau^{\prime}\right) c_{p k_{4}}\left(\tau^{\prime}\right) c_{p k_{1}}^{+}(\tau) c_{s k_{2}}(\tau)\right| n\right\rangle e^{-\beta E_{n}}
\end{gathered}
$$

Os sistemas Metal hospedeiro e ponta de prova metálica (descritos pelo Hamiltoniano de Anderson e pelo Hamiltoniano do gás de elétrons livres respectivamente) são independentes, ou seja, $Z=Z_{A} Z_{P}$, onde $Z_{A}$ e $Z_{P}$ são funções de partição do Essemble Grã-Canônico relativos ao Hamiltoniano de Anderson e da ponta de prova metálica respectivamente. Usamos também as identidades $\sum_{m}|m\rangle\langle m|=1$ e $|m\rangle=\left|f^{\prime}\right\rangle\left|g^{\prime}\right\rangle$, 
A conservação da energia da distribuição Delta de Dirac, dá

$$
\begin{gathered}
I=-\frac{2 \pi e t^{2}}{\hbar} \sum_{f f, k} Z_{A}^{-1} e^{-\beta E_{f}^{A}}\left|\sum_{q \sigma}\left\langle f\left|e^{i \vec{q} \cdot \overrightarrow{R_{2}}} c_{s q \sigma}\right| f^{\prime}\right\rangle\right|^{2}\left(e^{-\beta \mu_{P}}-1\right) f\left(\varepsilon_{k}^{P}-\mu_{P}\right) \times \\
\times \delta\left(E_{f}^{A}-E_{f}^{A}+\varepsilon_{k}^{P}\right) .
\end{gathered}
$$

A diferença de potencial elétrico $\phi$ é muito pequena, e por isso trabalharemos em regime linear, ou seja, $e^{-\beta \mu_{P}} \cong 1-\beta \mu_{P}=1-\beta e \phi$ e $f\left(\varepsilon_{k}^{P}-\mu_{P}\right) \cong f\left(\varepsilon_{k}^{P}\right)-$ $\phi \frac{\partial}{\partial \varepsilon_{k}^{P}} f\left(\varepsilon_{k}^{P}\right)$.

Utilizando-se de mais algumas identidades

$$
\begin{aligned}
& e^{-\beta E_{f}^{A}} \delta\left(E_{f}^{A}-E_{f}^{A}+\varepsilon_{k}^{p}\right)=\left(1-f\left(\varepsilon_{k}^{p}\right)\right)\left(e^{-\beta E_{f}^{A}}+e^{-\beta E_{f}^{A}}\right) \times \\
& \times \delta\left(E_{f}^{A}-E_{f}^{A}+\varepsilon_{k}^{p}\right) \\
& f\left(\varepsilon_{k}^{p}\right) \times\left(1-f\left(\varepsilon_{k}^{p}\right)\right)=-\frac{1}{\beta} \frac{\partial}{\partial \varepsilon_{k}^{p}} f\left(\varepsilon_{k}^{p}\right)
\end{aligned}
$$

Assim, chega-se a uma expressão de corrente até primeira ordem em $\phi$,

$$
\begin{aligned}
I=\frac{2 \pi e^{2} t^{2}}{\hbar} \phi \sum_{k f f} Z_{A}^{-1}\left|\sum_{q \sigma}\left\langle f\left|e^{i \vec{q} \cdot \overrightarrow{R_{2}}} c_{s q \sigma}\right| f^{\prime}\right\rangle\right|^{2}\left(e^{-\beta E_{f}^{A}}+e^{-\beta E_{f}^{A},}\right) \times & \\
& \times\left(-\frac{\partial}{\partial \varepsilon_{k}^{p}} f\left(\varepsilon_{k}^{p}\right)\right) \quad \delta\left(E_{f}^{A}-E_{f}^{A}+\varepsilon_{k}^{p}\right) .
\end{aligned}
$$


Para o caso da função de Green avançada, temos

$$
\begin{array}{r}
G_{a b}^{A}(t)=\frac{i}{\hbar} \theta(-t) \sum_{N} Z^{-1} e^{-\beta E_{N}}\left\langle N\left|\left[a^{+}(0), b(t)\right]_{+}\right| N\right\rangle= \\
=\frac{i}{\hbar} \theta(-t) \sum_{N, M} Z^{-1}\left\langle M\left|a^{+}\right| N\right\rangle\langle N|b| M\rangle\left(e^{-\beta E_{N}}+e^{-\beta E_{M}}\right) e^{\frac{i}{\hbar}\left(E_{N}-E_{M}\right) t}
\end{array}
$$

A transformada de Fourier para este caso é

$G_{a b}^{A}(\varepsilon)=\int G_{a b}^{A}(t) e^{\frac{i}{\hbar}(\varepsilon-i \delta) t} d t=\sum_{N, M} Z^{-1}\left\langle M\left|a^{+}\right| N\right\rangle\langle N|b| M\rangle \frac{\left(e^{-\beta E_{N}}+e^{-\beta E_{M}}\right)}{\varepsilon+E_{N}-E_{M}-i \delta}$.

A transformada sobre $\frac{\partial}{\partial t} G_{a b}^{A}(t)$ é

$$
\int \frac{\partial}{\partial t} G_{a b}^{A}(t) e^{\frac{i}{\hbar}(\varepsilon-i \delta) t} d t=-\frac{i}{\hbar}(\varepsilon-i \delta) G_{a b}^{A}(\varepsilon)
$$

Concluímos portanto

$$
G_{a b}^{R}(\varepsilon)=\left(G_{a b}^{A}(\varepsilon)\right)^{+}
$$

A seguir, mostraremos alguns passos algébricos que permitirão escrever a condutância em uma forma mais adequada para cálculo.

Considere primeiramente a função de Green retardada temporal

$$
G^{R}(t)=-\frac{i}{\hbar} \theta(t) \sum_{N} Z^{-1} e^{-\beta E_{N}}\left\langle N\left|\left[O(t), O^{+}(0)\right]_{+}\right| N\right\rangle
$$

onde

$$
O=\sum_{k, \sigma} e^{i \vec{k} \cdot \vec{R}_{2}} c_{s k \sigma}
$$


O procedimento para determinar o comportamento explícito de $G_{k k}^{R}(\varepsilon)$ e $G_{k q}^{R}(\varepsilon)$ é análogo.

Sabemos que

$$
G_{k q}^{R}(t)=-\frac{i}{\hbar} \theta(t) \sum_{N} Z^{-1} e^{-\beta E_{N}}\left\langle N\left|\left[c_{s k}(t), c_{s q}^{+}(0)\right]_{+}\right| N\right\rangle
$$

e

$$
\begin{aligned}
\frac{\partial}{\partial t} G_{k q}^{R}(t)= & -\frac{i}{\hbar} \delta(t) \sum_{N} Z^{-1} e^{-\beta E_{N}}\left\langle N\left|\left[c_{s k}(t), c_{s q}^{+}(0)\right]_{+}\right| N\right\rangle+ \\
& -\frac{i}{\hbar} \theta(t) \sum_{N} Z^{-1} e^{-\beta E_{N}}\left\langle N\left|\left[\frac{\partial}{\partial t} c_{s k}(t), c_{s q}^{+}(0)\right]_{+}\right| N\right\rangle,
\end{aligned}
$$

ou seja,

$$
\frac{\partial}{\partial t} G_{k q}^{R}(t)=-\frac{i}{\hbar} \delta(t) \sum_{N} Z^{-1} e^{-\beta E_{N}}\left\langle N\left|\left[c_{s k}(t), c_{s q}^{+}(0)\right]_{+}\right| N\right\rangle+\frac{i}{\hbar} \varepsilon_{k}^{s} G_{k q}^{R}(t)+\frac{i}{\hbar} V G_{d q}^{R}(t) .
$$

Lembrando que $G_{d q}^{R}(\varepsilon)=\left(G_{d q}^{A}(\varepsilon)\right)^{+}$, basta então determinar $G_{d q}^{A}(\varepsilon)$.

A função de Green que precisamos é

$$
G_{d q}^{A}(t)=\frac{i}{\hbar} \theta(-t) \sum_{N} Z^{-1} e^{-\beta E_{N}}\left\langle N\left|\left[c_{d}^{+}(0), c_{s q}(t)\right]_{+}\right| N\right\rangle .
$$

Fazendo a derivada parcial com relação ao tempo, obtemos

$$
\begin{aligned}
\frac{\partial}{\partial t} G_{d q}^{A}(t) & =-\frac{i}{\hbar} \delta(t) \sum_{N} Z^{-1} e^{-\beta E_{N}}\left\langle N\left|\left[c_{d}^{+}(0), c_{s q}(t)\right]_{+}\right| N\right\rangle+ \\
& +\frac{i}{\hbar} \theta(-t) \sum_{N} Z^{-1} e^{-\beta E_{N}}\left\langle N\left|\left[c_{d}^{+}(0), \frac{\partial}{\partial t} c_{s q}(t)\right]_{+}\right| N\right\rangle .
\end{aligned}
$$

No espaço de energia essa função fica finalmente igual à 
Seridonio, Antonio Carlos Ferreira.

Influência do Efeito Kondo na condutância de contatos pontuais de superfícies metálicas/Antonio Carlos Ferreira Seridonio.-São Carlos, 2002.

$82 \mathrm{p}$.

Carlos,

Dissertação (Mestrado) - Instituto de Física de São 2002.

Orientador: Prof. Dr. Luiz Nunes de Oliveira

1. Metais. 2. Modelo de Anderson. 3. Tunelamento eletrônico. I. Título. 
A minha mãe Maria Eugênia, pela formação de meu caráter. 
Este trabalho foi financiado pela Fundação de Amparo à Pesquisa do Estado de São Paulo (FAPESP) 


\section{AGRADECIMENTOS}

- Ao Prof. Dr Luiz Nunes de Oliveira, meu orientador, por ter ensinado que as grandes idéias sobre Física nascem de pensamentos transparentes e criativos.

- Ao Prof. Dr. Makoto Yoshida, pelo suporte matemático e pelas compreensões profissional e pessoal, por meio das quais as grandes idéias sobre Física puderam ser concretizadas.

- A todos os amigos do Grupo pelas sugestões diretas ou indiretas que auxiliaram no entendimento do Grupo de Renormalização Numérico.

- Aos professores Maria Ivone e José Caluri, Edson e Erick, professores de Matemática e Física do ensino médio, pela iniciação sólida nas Ciências Exatas.

- Aos professores da UNESP, pela formação de graduação que permitiu meu crescimento profissional nestes estudos.

- Aos amigos Antonio Joaquim, Rodrigo Seraide, Paulo de Castro e Kerson Rocha pelo empréstimo de seus tolerantes ouvidos aos relatos que fiz sobre minhas incertezas durante o desenrolar do projeto, sem os quais não teria iniciado o aprendizado sobre sua oratória.

- A Pousada Sakura pelo oferecimento de um ambiente adequado para leitura, onde pude dedicar-me aos estudos de livros e artigos ligados a esta dissertação.

- Aos meus pais Maria Eugênia e José Maria, pelo inestimável investimento afetivo em minhas formações prossional e pessoal que resultaram no sucesso de todas as realizações e conquistas aqui citadas.

- A Deus, pela perfeita seleção de pessoas inserida em minha vida que propiciou o cumprimento de toda esta atividade. 


\section{Resumo}

A microscopia de varredura por tunelamento (MVT) é uma nova maneira de se observar experimentalmente o efeito Kondo. Quando uma concentração de átomos é adicionada a um meio metálico (metal hospedeiro), a corrente de tunelamento passa a depender de fatores de origem não geométrica. O rearranjo das cargas dentro do volume metálico (oscilações de Friedel) e o espalhamento de spins eletrônicos (efeito Kondo), devido a introdução de impurezas, mudam o valor da corrente e influenciam o levantamento da topografia do espécime examinado. Esses fatores devem ser considerados para que a topografia gerada seja condizente com a topografia verdadeira. Utilizamos como modelo teórico para descrição desse sistema, o modelo de Anderson de uma impureza para simular o espécime examinado e uma banda de condução livre para representar os elétrons da agulha metálica do microscópio. Nossa abordagem usa a fórmula de Kubo para o cálculo da corrente de tunelamento, supondo o Hamiltoniano de tunelamento como perturbação e o potencial elétrico no regime linear. Apresentamos inicialmente um estudo para o Modelo do Nível Ressonante, isto é, o modelo de Anderson sem correlação, com o objetivo de demonstrar a precisão do método do Grupo de Renormalização Numérico. Em seguida, analisamos o Modelo de Anderson correlacionado. Os resultados tanto para a condutância em função da distância entre ponta e impureza a temperatura fixa, como para a condutância em função da temperatura a distância fixa, permitem interpretação física transparente desde que levem em conta a ressonância de Kondo na densidade espectral. 


\begin{abstract}
The scanning tunneling microscopy (STM) is a new way to observe experimentally the Kondo effect. When a concentration of atoms is added to a sample (host metal), the tunelling current begins to depend on other non-geometric factors. The rearrangement of charges in the metallic bulk (Friedel oscillations) and the electronic spin scattering (Kondo effect), due to the presence of impurities, change the current value and affect the sample's topography. These factors must be considered in order to make a correspondence between the generated topography with the true one. As a theoretical description of the system, we use the single impurity Anderson model to simulate the examined sample and a free conduction band to represent the electrons of the microscope metallic tip. Our treatment uses the Kubo formula to calculate the tunneling current, assuming the tunneling Hamiltonian as a perturbation and the electric potencial in the linear regime. We iniatially present a study of the Ressonant Level Model, i.e, the Anderson model without correlation, to show the accuray of the Numerical Renormalization Group procedure. In the next step, we analyse the correlated Anderson model. The dependence of the conductance on tip-impurity distance, at constant temperature, and its dependence on temperature for constant tip-impurity distance, allow a clear physical interpretation after taking into account the Kondo ressonance in the spectral density.
\end{abstract}




\section{Sumário}

1 Introdução 1

2 Modelo de Anderson $\quad 6$

2.1 O Hamiltoniano de Anderson . . . . . . . . . . . . . . 6

2.2 Regimes do Modelo de Anderson . . . . . . . . . . . . . . . 10

3 Fórmula de Kubo para a condutância $\quad 13$

3.1 Funções de Green no cálculo da condutância . . . . . . . . . . . . 19

4 Grupo de Renormalização Numérico 24

4.1 Discretização Logarítmica . . . . . . . . . . . . . . . . 24

4.2 Tridiagonalização do Hamiltoniano de Condução ...... 25

4.3 Truncamento . . . . . . . . . . . . . . . . . . . 28

4.4 Estudo de Pontos Fixos . . . . . . . . . . . . . . . . . . 30

5 Modelo do Nível Ressonante $\quad 34$

5.1 Fórmula exata da condutância . . . . . . . . . . . . . 35

5.2 Fórmula da Condutância via GRN . . . . . . . . . . . . 36

5.3 Resultados . . . . . . . . . . . . . . . . . . . . 41

6 Caso Interagente $\quad 52$

6.1 Diagonalização Iterativa ～. . . . . . . . . . . . . . . . . . 52

6.2 Fórmula da condutância para o caso interagente . . . . . . . 58

6.3 Resultados . . . . . . . . . . . . . . . . . . . 59 
7 Conclusões

A Resultados importantes para a fórmula de Kubo

B Funções de Green a temperatura $T$ no cálculo da fórmula de Kubo

C Cálculo de integrais

78 


\section{Lista de Figuras}

1.1 Esquema simplificado do aparato do experimento de STM. São mostradas uma ponta de prova metálica (haste com uma ponta triangular azul) e uma superficie metálica (Metal hospedeiro) contendo uma impureza atômica adsorvida (círculo vermelho) submetidos a uma pequena diferença de potencial elétrico $\phi$ (regime linear) . O ket $|d\rangle$ representa o orbital da impureza atômica que participa do espalhamento com os elétrons da banda de condução.

2.1 Representação do termo de hibridização V do Modelo de Anderson. É mostrado num esquema simplificado (sem spin). Primeiramente o espalhamento de um elétron do nível de energia do orbital de impureza para a banda de condução e em seguida o espalhamento de um elétron da banda de condução para esse nível. Os parâmetros $D$ e $\varepsilon_{F}$ são a meia largura de banda e o nível de Fermi respectivamente. . . . . . . . . . . . . . .

2.2 Distribuição de níveis de energia dos autoestados do Hamiltoniano de impureza. Existem três possíveis configurações de energia quando a impureza está desacoplada da banda de condução . Aconfiguração (i) não possui nenhum elétron e tem energia $E_{0}=0$. A configuração (ii) está com um único elétron e tem energia $E_{1}=\varepsilon_{d}$. A configuração (iii) é a configuração em que ambos os níveis estão ocupados e possui a energia $E_{2}=2 \varepsilon_{d}+U \ldots \ldots$. . . . . 
2.3 Densidade espectral $\rho_{f}$ para o modelo de Anderson com correlação $U$. As curvas foram geradas via Grupo de Renormalização Numérico assumindo $\varepsilon_{d}=-0.01 D$ e $\Gamma=0,01 D$. Foram usados os parâmetros $U=0,05 D, U=0,105 D$ e $U=0,15 D$ respectivamente. A linha sólida é uma combinação de Lorentzianas para ajustar os pontos de densidade criados numericamente. Quando $U \rightarrow 0$, a densidade espectral de impureza se aproxima de uma Lorentziana com meia largura igual à $\Gamma$ e centrada em $\varepsilon_{d}$. . . . .

4.1 Discretização logarítmica, com $\Lambda^{-(m+z)}<|k|<\Lambda^{(1-z-m)}$ e $m=$ $1,2, \ldots, \Lambda>1$ e $0<z \leq 1$.O índice $m$ é um índice de intervalo dentro do espaco $k$ e $\Lambda$ é a razão entre dois valores consecutivos de energia. O limite $\Lambda \rightarrow 1$ recupera o espectro contínuo de energias e a discretização original [4] é obtida fazendo-se $z=1$.

5.1 Densidade Espectral de Impureza contra energia com $\varepsilon_{d}=0,1 D$ e

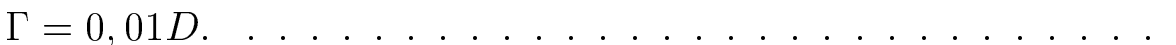

5.2 Evolução de um autovalor com N. A curva vermelha se refere ao segundo autovalor para $\mathrm{N}$ ímpar $(\lambda)$ e a curva azul para $\mathrm{N}$ par $(\eta)$. Nas proximidades de $N=20$ o autovalor $\lambda$ ( $N$ ímpar) se comporta como ponto fixo de $N$ par. Os parâmetros usados foram $\Lambda=3$, $V=0,05 D$ e $\varepsilon_{d}=0$

5.3 O gráfico compara a curva gerada considerando a parte real da função de Green associada à impureza $G_{d d}(\varepsilon)$ (preta) com aquela desconsiderando a parte real (vermelha). Os parâmetros utilizados foram $V=t=0,01 D, \varepsilon_{d}=-0,01 D$ e $q_{F} R=\pi$. Esse resultado confirma a pequena influência da parte real da função de Green na condutância conforme, adiantado na seção 5.1 . . . . . . . . . . 42 
5.4 Oscilações da condutância a baixas temperaturas nos casos $\mathrm{z}=0,5$ (curva preta) e $\mathrm{z}=1,0$ (curva vermelha). A condutância verdadeira (curva verde) é a média entre as condutâncias com $z=0,5$ e com $z=1,0$. Os parâmetros utilizados foram $N=25, \Lambda=3$, $V=t=0,01 D, \varepsilon_{d}=-0,01 D$ e $q_{F} R=\pi \ldots \ldots \ldots$

5.5 O gráfico compara a curva azul gerada pelo Grupo de Renormalização Numérico ( $N=25$ e $\Lambda=3$ ) com a fórmula exata (curva vermelha) usando $V=t=0,01 D, \varepsilon_{d}=-0,01 D$ e $q_{F} R=\pi$. Em ambos os casos, $\Re\left\{G_{d d}(\varepsilon)\right\}$ foi negligenciada. Esse gráfico mostra que a generalização da técnica de Grupo de Renormalização para cálculo de propriedades termodinâmicas produz resultados exce-

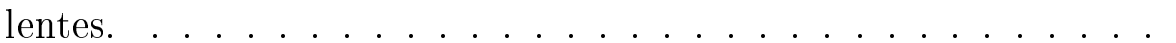

5.6 O gráfico compara dois casos para dois valores de $\varepsilon_{d}$, respectivamente $\varepsilon_{d}=0$ (curva azul) e $\varepsilon_{d}=-0,001 D$ (curva vermelha). A curva vermelha apresenta um pico nas proximidades do nível de energia $\left|\varepsilon_{d}\right|$, onde a densidade espectral de impureza é a pricipal responsável por este máximo. A curva azul não possui um máximo em temperaturas intermediárias, mas tem uma patamar intenso a baixas temperaturas. Os parâmetros usados foram $V=$ $t=0,01 D, q_{F} R=\pi, N=25$ e $\Lambda=6 \ldots \ldots \ldots$

5.7 Gráfico da condutância contra distância com $k_{B} T=10^{-6} D$ gerado numericamente. Os parâmetros usados foram $N=25, \Lambda=3$, $V=t=0,01 D, \varepsilon_{d}=-0,01 D$. A oscilação da corrente em função da distância é uma das consequências das oscilações de Friedel. . .

5.8 Gráfico da condutância contra distância com $k_{B} T=10^{-6} D$ gerado numericamente. Os parâmetros usados foram $N=25, \Lambda=3$, $V=t=0,01 D$ e $\varepsilon_{d}=0$. A oscilação da corrente em função da distância é uma das consequências das oscilações de Friedel. . . . 
6.1 Densidade espectral $\rho_{f}$ para o modelo de Anderson com correlação $U$. As curvas foram geradas via Grupo de Renormalização Numérico assumindo $\varepsilon_{d}=-0.01 D$ e $\Gamma=0,01 D$. Foram usados os parâmetros $U=0,05 D, U=0,105 D$ e $U=0,15 D$ respectivamente. A linha sólida é uma combinação de Lorentzianas para ajustar os pontos de densidade criados numericamente. Quando $U \rightarrow 0$, a densidade espectral de impureza se aproxima de uma Lorentziana com meia largura igual à $\Gamma$ e centrada em $\varepsilon_{d}$. . . . .

6.2 A curva vermelha apresenta um condutância que decai com o aumento de temperatura e que aumenta drasticamente com a diminuição da temperatura até um valor constante. Apresenta também um máximo local num intervalo de tempertaturas intermediárias. A curva azul possui comportamento semelhante. Em ambos os casos, a alta condutância se deve à ressonância de Kondo. Os parâmetros utilizados foram $\varepsilon_{d}=-0,01 D, U=0,02 D, q_{F} R=\pi, \Lambda=6 \mathrm{e}$ $N=46 \ldots \ldots \ldots \ldots \ldots \ldots \ldots \ldots \ldots$

6.3 A curva vermelha mostra que a correlação $U=0,01 D \operatorname{com} \varepsilon_{d}=$ $-0,01 D$ faz com a condutância se comportar com se não existisse correlação e se o nível de energia de impureza tivesse um valor normalizado. A curva azul também manifesta uma forma similar do Modelo do Nível Ressonante. Os parâmetros do GRN usados foram $\Lambda=6$ e $N=46$

6.4 Condutância em função da distância numa temperatura próxima de zero para três casos interagentes. Vê-se que as amplitudes de oscilação diminuem com a diminuição do parâmetro de correlação $U$. Os parâmetros usados nas três curvas foram $\varepsilon_{d}=-0,01 D$, $V=0,02 D, \Lambda=6$ e $N=32 \ldots \ldots \ldots \ldots$ 


\section{Lista de Tabelas}

4.1 Autovalores de energia para $\mathrm{N}$ ímpar. . . . . . . . . . . . . . . . 31

4.2 Autovalores de energia para $\mathrm{N}$ par . . . . . . . . . . . . 32 


\section{Capítulo 1}

\section{Introdução}

O problema de Kondo é um dos poucos problemas do magnetismo em metais onde a manifestação de efeitos de muitos corpos é bem compreendida. Consequentemente, esse problema tem sido a base para o estudo de sistemas mais complexos como os metais de valen̂cia flutuante e sistemas eletrônicos fortemente correlacionados como os metais de férmions pesados. A manifestação do efeito Kondo tem sido comprovada pela concordância entre cálculos teóricos de grandezas termodinâmicas como o calor específico, susceptibilidade magnética e de propriedades dinâmicas como a densidade espectral de sistemas compostos por impurezas magnéticas diluídas em meios metálicos e os resultados experimentais.

Esse quadro foi enriquecido recentemente com a observação experimental do efeito Kondo em um novo contexto; o de impurezas magnéticas adsorvidas quimicamente em superfícies metálicas. A técnica de medida utilizada foi a STM (Scanning Tunneling Microscopy) [1], uma das variações da microscopia eletrônica cujo poder de resolução na escala atômica tem permitido, além da construção de imagens topográficas de adsorvidos em superfícies metálicas, a caracterização da estrutura eletrônica do adsorvido e do substrato. Grosso modo, a aplicação dessa técnica consiste em medir a corrente de tunelamento através de um contato que se estabelece quando uma ponta metálica é colocada nas proximidades da superfície do substrato ou nas proximidades de uma impureza adsorvida nesse substrato. 
Dentro desse contexto, o efeito Kondo foi observado experimentalmente em medidas de corrente de tunelamento através de um átomo de Ce adsorvido em superfície de Ag.

A interpretação da imagem topográfica tem sido problemática e muitas vezes a imagem obtida não corresponde à imagem topográfica real. A origem desse problema é que a corrente de tunelamento não depende apenas de fatores geométricos, mas também da estrutura eletrônica do substrato e da haste metálica. Ela também pode depender da oscilação da densidade de cargas ao redor do contato pontual, da interferência entre os vários canais de tunelamento através do contato e do efeito Kondo. Todos esses efeitos interferem na corrente de tunelamento e introduzem características anômalas na imagem topográfica. Portanto, a interpretação correta da topografia depende diretamente de nossa capacidade de identificar e separar as características geométricas das contribuições dadas pelos processos eletrônicos no substrato e no contato.

Uma das motivações para a proposição deste trabalho é a constatação de que problemas que envolvem o espalhamento de elétrons por impurezas diluídas em meio metálico como o efeito Kondo e as oscilações de Friedel, já bastante estudados em outros contextos como o do magnetismo em sistemas eletronicamente correlacionados, passam a ser de grande importância em medidas de microscopia eletrônica em superfícies de sólidos. A segunda motivação é dar prosseguimento ao desenvolvimento da técnica do Grupo de Renormalização Numérico.

Estamos propondo este trabalho com o objetivo de investigar o efeito Kondo em superfícies metálicas e sua influência na corrente de tunelamento por meio de contatos pontuais implantados na superfície de um substrato metálico e nas vizinhanças de uma impureza magnética adsorvida nesta superfície. A possibilidade de medidas diretas do efeito Kondo através da técnica STM, torna bastante oportuno estudos dessa natureza.

Estudaremos o comportamento da corrente de tunelamento em regime linear no problema de STM (Scanning Tunelling Microscopy) entre dois sistemas 


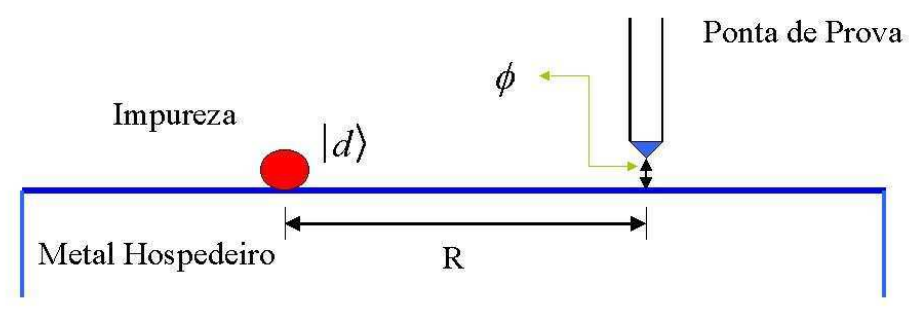

Figura 1.1: Esquema simplificado do aparato do experimento de STM. São mostradas uma ponta de prova metálica (haste com uma ponta triangular azul) e uma superficie metálica (Metal hospedeiro) contendo uma impureza atômica adsorvida (círculo vermelho) submetidos a uma pequena diferença de potencial elétrico $\phi$ (regime linear) . O ket $|d\rangle$ representa o orbital da impureza atômica que participa do espalhamento com os elétrons da banda de condução.

metálicos. O primeiro consiste num metal com uma impureza atômica adsorvida, descrito pelo modelo de Anderson. O segundo é uma banda livre de condução representada pela ponta de prova metálica do microscópio eletrônico. Essa ponta metálica, podendo mover-se livremente por todo o metal fica situada acima da superfície do metal hospedeiro, distando uma altura fixa, na qual existe um diferença de potencial $\phi$. A medida de corrente elétrica no experimento de STM consiste em deslocar a ponta lateralmente em relação à impureza atômica e verificar como a distância entre o ponto onde se dá o tunelamento e a impureza influencia a corrente. Podemos também nos perguntar qual deverá ser o comportamento dessa corrente sem o deslocamento da ponta, mas com a temperatura variável. Experimentalmente, a corrente de tunelamento é medida indiretamte, a partir da condutância diferencial $\mathrm{G}$, que consisite em medir a razão entre a variação da corrente de tunelamento e a variação do potencial elétrico aplicado, isto é, $G=\frac{\partial I}{\partial \phi}$. O arranjo de STM é mostrado na Figura 1.1. 
O estudo da corrente de tunelamento entre um metal com uma impureza adsorvida e uma ponta de prova metálica deve ser sistematizado. Primeiramente, é preciso entender a Física que existe na interação de elétrons de uma banda de condução metálica com uma impureza adicionada ao volume metálico. Para isso deve ser descrita por um Hamiltoniano capaz de estabelecer os mecanismos significativos de interação presentes em sistema metálicos com impurezas, como por exemplo, estabelecer como se dá o espalhamento entres os elétrons da banda de condução por uma impureza, seja essa magnética ou não, e também ser capaz de incluir correlação eletrônica no sítio de impureza. O Hamiltoniano típico para o estudo de propriedades termodinâmicas de sistemas metálicos, envolvendo os mecanismos citados, é o Hamiltoniano de Anderson, cuja apresentação e descrição, assim como os regimes de formação de momentos magnéticos localizados são discutidos no capítulo 2.

A descrição do Hamiltoniano de Anderson ainda não caracteriza o problema de STM por completo, pois é preciso estabelecer uma ligação entre os elétrons do metal hospedeiro e os elétrons da ponta de prova metálica do microscópio eletrônico. É essa ligação que promove o tunelamento entre elétrons dos dois sistemas metálicos. No capítulo 3, apresentamos uma dedução formal da fórmula de Kubo que inclui em sua formulação a hipótese de regime linear no potencial elétrico $\phi$. Usamos também funções de Green de muitos corpos a temperatura $T$ para simplificar e escrever a condutância na representação de Lehmann. Essa forma, como veremos no capítulo 5 , é adequada para a posterior implementação do Grupo de Renormalização Numérico.

No capítulo 4, apresentamos o método do Grupo de Renormalização Numérico, indroduzindo a discretização logarítmica da banda de condução e a tranformação do Hamiltoniano de Anderson de uma base contínua e infinita para uma discreta e finita, gerada a partir da construção Lanczos. Desenvolvemos também um estudo de pontos fixos, ilustrando com o exemplo da banda livre de condução.

No capítulo 5, particularizamos o Hamiltoniano de Anderson ao Modelo do 
Nível Ressonante, que é aquele que negligencia a correlação eletrônica U no sítio de impureza. O Modelo do Nível Ressonante permite uma diagonalização exata e por isso dispensa qualquer abordagem numérica. O objetivo principal desse capítulo é, além de discutir e interpretar o comportamento da condutância devido a introdução de uma impureza não magnética num meio metálico, seja em função da temperatura ou em função da distância, confrontar a precisão entre a fórmula exata da condutância de contato com a obtida via Grupo de Renormalização Numérico.

O capítulo 6 é voltado ao caso com correlação eletrônica no sítio da impureza, com a apresentação do método de diagonalização iterativa para o Hamiltoniano de Anderson. Da mesma forma que no estudo do Modelo do Nível Ressonante, apresentamos a dependência da condutância com a temperatura e também com a distância entre a ponta de prova e a impureza adsorvida.

No capítulo 7 apresentamos nossas conclusões sobre este trabalho. 


\section{Capítulo 2}

\section{Modelo de Anderson}

O modelo de Anderson foi idealizado e introduzido com a finalidade de se estudar as propriedades de sistemas constituídos de impurezas magnéticas diluídas em metais não magnéticos [2]. Posteriormente, foi utilizado no estudo de propriedades dinâmicas e termodinâmicas de compostos de valência flutuante ou compostos de terras raras em sistemas metálicos e no estudo de propriedades dinâmicas como o espectro de fotoemissão eletrônica de impurezas adsorvidas em superfícies metálicas, como é o caso neste trabalho, onde o modelo de Anderson será utilizado para descrever uma impureza adsorvida em superfícies de metais. Vale ainda destacar um outro aspecto importante envolvendo o modelo de Anderson: ele tem sido intensivamente estudado nas últimas décadas com a finalidade de desenvolvimento de novas técnicas que possibilitem o estudo do efeito de muitos corpos em propriedades dinâmicas e termodinâmicas de sistemas eletronicamente correlacionados.

\subsection{O Hamiltoniano de Anderson}

O modelo de Anderson, que descreve um gás de elétrons livres na presença de um nível de energia, é adequado para o estudo de propriedades físicas de metais com impurezas magnéticas. 
Este modelo escrito em linguagem de segunda quantização apresenta em seu Hamiltoniano quatro termos que sintetizam as interações dos elétrons de uma banda de condução de um metal com impurezas magnéticas adicionadas. Possui a energia cinética do gás não perturbado e a do mencionado nível de energia. Inclui também um mecanismo de espalhamento eletrônico entre a banda de condução e o momento magnético localizado da impureza, que pode ser representado por um elétron num orbital $d$ de um elemento de transição ou $f$ de uma terra rara. Supomos que esse nível de energia, que representa a impureza possui apenas degenerescência de spin. Quando ele estiver duplamente ocupado, existirá uma repulsão coulombiana entre esses estados eletrônicos localizados.

O Hamiltoniano de Anderson é

$$
H=H_{e l}+H_{d}+H_{c o r r}+H_{s d}
$$

onde o termo

$$
H_{e l}=\sum_{k \sigma} \epsilon_{k} c_{k \sigma}^{+} c_{k \sigma}
$$

representa o operador energia cinética do gás de elétrons livres. O parâmetro $\epsilon_{k}$ é a energia cinética de um elétron com momento $k$ da banda de condução, $c_{k \sigma}^{+}$ e $c_{k \sigma}$ são operadores de criação e destruição de um elétron da banda de condução com momento $k$ e spin $\sigma$.

O termo

$$
H_{d}=\varepsilon_{d}\left(c_{d \uparrow}^{+} c_{d \uparrow}+c_{d \downarrow}^{+} c_{d \downarrow}\right)
$$

é a energia não perturbada dos estados d da impureza. Os operadores $c_{d \sigma}^{+} \mathrm{e}$ $c_{d \sigma}$ são operadores de criação e destruição de um elétron no nível $\varepsilon_{d}$ com spin $\sigma$.

O terceiro termo do Hamiltoniano é aquele que considera a repulsão coulombiana no sítio da impureza, ou seja, existe uma energia elétrica de repulsão $U$ 
quando o sítio da impureza estiver duplamente ocupado. Essa correlação é representada por

$$
H_{c o r r}=U c_{d \uparrow}^{+} c_{d \uparrow} c_{d \downarrow}^{+} c_{d \downarrow}
$$

A repulsão coulombiana pode ser calculada como

$$
U=\int\left|\varphi_{d}\left(\overrightarrow{r_{1}}\right)\right|^{2} \frac{e^{2}}{\left|\overrightarrow{r_{1}}-\overrightarrow{r_{2}}\right|}\left|\varphi_{d}\left(\overrightarrow{r_{2}}\right)\right|^{2} d V_{1} d V_{2}
$$

onde $\varphi_{d}\left(\overrightarrow{r_{1}}\right)$ e $\varphi_{d}\left(\overrightarrow{r_{2}}\right)$ são as auto-funções dos eletrons localizados na impureza.

Nesse modelo desprezamos a correlação entre estados eletrônicos localizados e da banda de condução, pois a função de onda dos estados ligados é relevante somente nas vizinhanças da camada $|d\rangle$ da impureza.

Finalmente o quarto e último termo apresenta um mecanismo de espalhamento que permite arrancar um elétron da banda de condução para o nível de energia localizado, ou arrancar um elétron localizado e jogá-lo na banda de condução. Os elétrons da banda são, na verdade, quase livres e pertencem aos orbitais s dos átomos que formam o metal. Esse termo é

$$
H_{s d}=\sum_{k \sigma} V_{d k}\left(c_{s k \sigma}^{+} c_{d \sigma}+c_{d \sigma}^{+} c_{s k \sigma}\right)
$$

onde $V_{d k}$ é o termo de hibridização que acopla a banda de condução com a impureza.

Para metais, é suficiente assumir $V_{d k}=V$ como constante, pois a blindagem local de estados diminui a importância da dependência do momento envolvido no espalhamento de elétrons da banda de condução. O mecanismo representado pelo termo $H_{s d}$ é ilustrado na Figura 2.1. 

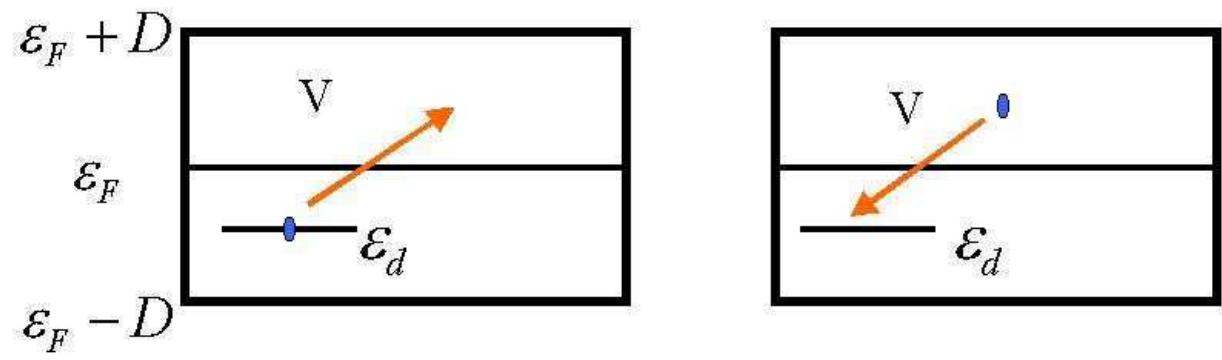

$\varepsilon_{F}+D$
$\varepsilon_{F}$
$\varepsilon_{F}-D$

Figura 2.1: Representação do termo de hibridização V do Modelo de Anderson. É mostrado num esquema simplificado (sem spin). Primeiramente o espalhamento de um elétron do nível de energia do orbital de impureza para a banda de condução e em seguida o espalhamento de um elétron da banda de condução para esse nível. Os parâmetros $D$ e $\varepsilon_{F}$ são a meia largura de banda e o nível de Fermi respectivamente. 


\subsection{Regimes do Modelo de Anderson}

O modelo pode ser bem compreendido em alguns regimes específicos estabelecidos por valores apropriados dos parâmetros $\varepsilon_{d}, \Gamma=\pi \rho\left(\varepsilon_{F}\right) V^{2}\left(\rho\left(\varepsilon_{F}\right)\right.$ é a densidade de estados avaliada no nível de Fermi), $U$ e pela temperatura $T$. Consideremos o regime de baixas temperaturas. O regime básico, a partir do qual podemos entender o modelo, origina-se no limite $\Gamma \rightarrow 0$, quando a impureza encontra-se desacoplada da banda de condução. Os autoestados da impureza podem ser classificados segundo os autovalores de $n_{d, \sigma}=d_{\sigma}^{\dagger} d_{\sigma}$ dados por 0,1 e 2 . As energias correspondentes são dadas por $E_{0}=0, E_{1}=\varepsilon_{d}$ e $E_{2}=2 \varepsilon_{d}+U$. Alternativamente, podemos ver os autoestados da impureza através de sua representação em um diagrama de níveis de energias de elétrons independentes. O primeiro nível possui energia $\varepsilon_{d}$ e o segundo $\varepsilon_{d}+U$, conforme ilustrado na Figura 2.2. O autoestado $n_{d}=0$ é dado pelo estado onde ambos níveis de energia estão desocupados; o autoestado $n_{d}=1$ corresponde ao estado em que o nível de energia $\varepsilon_{d}$ se encontra ocupado e finalmente, o autoestado $n_{d}=2$ corresponde ao caso em que ambos os níveis estão ocupados.

No caso em que $\Gamma \neq 0$, o orbital de impureza acopla-se à banda de condução. A ocupação do orbital de impureza não é mais número inteiro uma vez que elétrons da impureza podem passar para a banda de condução e vice-versa estabelecendo uma dinâmica de troca de carga que acaba por introduzir um alargamento nos níveis de energia da impureza. Entretanto, podemos avaliar qualitativamente a ocupação do nível de impureza em alguns casos limites dos parâmetros do modelo e da temperatura. Consideremos inicialmente o caso $\Gamma<<\left|\varepsilon_{d}\right|$ e $U \rightarrow \infty$. Neste caso o nível de energia $\varepsilon_{d}+U$ é inacessível e consequentemente o orbital de impureza pode estar desocupado ou singelamente ocupado. Porém, com $\varepsilon_{d}<\varepsilon_{F}$, o orbital deve estar ocupado com apenas um elétron configurando-se então a formação de momento magnético localizado na impureza. Este limite é conhecido como regime Kondo. Um outro regime é aquele estabelecido com $\left|\varepsilon_{d}\right|<<\Gamma$ e 


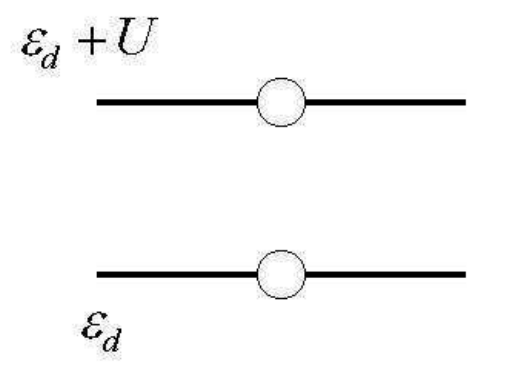

(i)

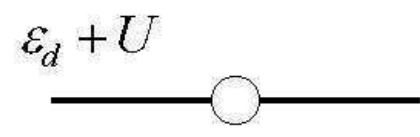

$\varepsilon_{d}+U$

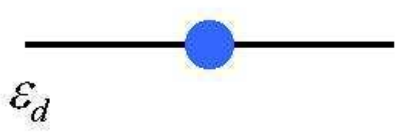

(ii)

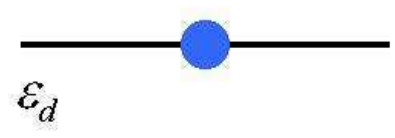

(iii)

Figura 2.2: Distribuição de níveis de energia dos autoestados do Hamiltoniano de impureza. Existem três possíveis configurações de energia quando a impureza está desacoplada da banda de condução . Aconfiguração (i) não possui nenhum elétron e tem energia $E_{0}=0$. A configuração (ii) está com um único elétron e tem energia $E_{1}=\varepsilon_{d}$. A configuração (iii) é a configuração em que ambos os níveis estão ocupados e possui a energia $E_{2}=2 \varepsilon_{d}+U$.

$U \approx \Gamma$, o estado duplamente ocupado também torna-se acessível, e o orbital de impureza fica parcialmente ocupado com dois elétrons. Neste caso, não se tem a formação de momento magnético.

A densidade espectral de impureza quando existe a correção eletrônica $U$, assume formas distintas que dependem do parâmetro $U$ empregado. A Figura 2.3 mostra algumas dessas formas.

Pode-se constatar que com o aumento da correlação U, começam a surgir máximos locais nas energias $\varepsilon_{d}$ e $\varepsilon_{d}+U$. O pico agudo na densidade espectral de impureza centrado no nível de Fermi é a ressonância de Kondo. 


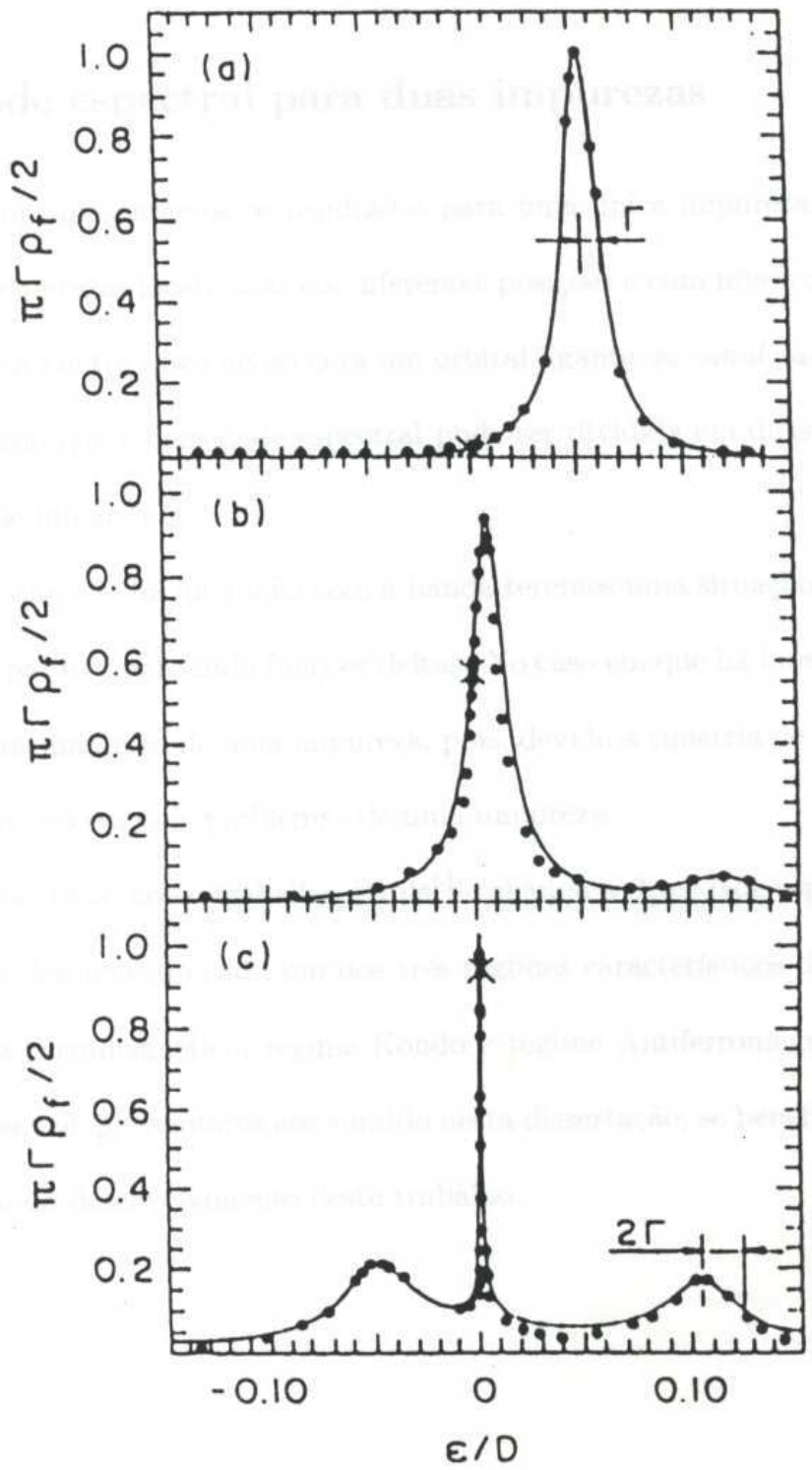

Figura 2.3: Densidade espectral $\rho_{f}$ para o modelo de Anderson com correlação $U$. As curvas foram geradas via Grupo de Renormalização Numérico assumindo $\varepsilon_{d}=-0.01 D$ e $\Gamma=0,01 D$. Foram usados os parâmetros $U=0,05 D, U=0,105 D$ e $U=0,15 D$ respectivamente. A linha sólida é uma combinação de Lorentzianas para ajustar os pontos de densidade criados numericamente. Quando $U \rightarrow 0$, a densidade espectral de impureza se aproxima de uma Lorentziana com meia largura igual à $\Gamma$ e centrada em $\varepsilon_{d}$. 


\section{Capítulo 3}

\section{Fórmula de Kubo para a}

\section{condutância}

Vamos efetuar um cálculo formal da corrente que flui através de um contato pontual em uma superfície metálica quando se estabelece uma diferença de potencial muito pequena através desse contato. Esta atividade pode ser considerada como o primeiro passo no desenvolvimento do projeto de pesquisa, que consiste em introduzir uma fórmua para a condutância no regime linear para o sistema físico que iremos estudar.

Começamos a trabalhar com problema de STM e a determinação da corrente de tunelamento no regime linear. O modelo teórico aqui empregado, é o que faz uso da fórmula de Kubo para sistemas a uma temperatura $T$. Considera-se que a ponta de prova metálica e o metal hospedeiro com a impureza atômica sejam sistemas idependentes fracamente acoplados por um Hamiltoniano de tunelamento, que faz o papel de perturbação no Hamiltoniano total.

O Hamiltoniano total $H_{o}$ na ausência de perturbação, isto é, quando não há aplicação de uma diferença de potencial é:

$$
H_{o}=H_{o}^{A}+H_{o}^{P}
$$




$$
\begin{array}{r}
H_{o}^{A}=H_{i}+H_{i s}+\sum_{k \sigma} \varepsilon_{k}^{s} c_{s k \sigma}^{+} c_{s k \sigma} \\
H_{i}=\sum \varepsilon_{d} c_{d \sigma}^{+} c_{d \sigma}+U c_{d \uparrow}^{+} c_{d \uparrow} c_{d \downarrow}^{+} c_{d \downarrow} \\
H_{i s}=V \sum_{k \sigma}\left(e^{-i \vec{k} \cdot \overrightarrow{R_{1}}} c_{s k \sigma}^{+} c_{d \sigma}+h . c .\right) \\
H_{o}^{P}=\sum_{k \sigma} \varepsilon_{k}^{p} c_{p k \sigma}^{+} c_{p k \sigma}
\end{array}
$$

Denotamos por $H_{o}^{A}$ o Hamiltoniano associado ao metal hospedeiro mais impureza (Hamiltoniano de Anderson). O Hamiltoniano relativo à ponta de prova metálica é identificado por $H_{o}^{P}$. Devemos entender os índices $s$ e $p$ como referentes aos operadores fermiônicos dos elétrons da superfície metálica e da ponta de prova respectivamente, enquanto que os operadores para o elétron da impureza atômica são denotados apenas por $c_{d \sigma}^{+}$e $c_{d \sigma}$, e o índice de spin é $\sigma$. A notação h.c. significa hermitiano conjugado. O parâmetro $U$ é a interação coulombiana entre dois elétrons no sítio de impureza. A função de $H_{i s}$ é acoplar a banda de condução do metal hospedeiro com a impureza atômica, localizada no sítio $\overrightarrow{R_{1}}$, através do parâmetro $V$. Nesse termo, fica claro que estamos usando como base ondas planas para representar os elétrons da banda de condução. O vetor $\overrightarrow{R_{2}}$ localiza a ponta de prova metálica, de modo que a distância entre a impureza atômica e a ponta de prova metálica é definida por $R=\left|\overrightarrow{R_{2}}-\overrightarrow{R_{1}}\right|$.

O termo responsável pela corrente de tunelamento através do contato pontual é o Hamiltoniano de tunelamento dado por

$$
H_{t}=t \sum_{k \sigma q \delta}\left(e^{-i \vec{k} \cdot \overrightarrow{R_{2}}} c_{p q \delta}^{+} c_{s k \sigma}+\text { h.c. }\right)
$$

O parâmetro t que aparece no Hamiltoniano de tunelamento acopla os sistemas metálicos.

Vamos introduzir os correspondentes potenciais químicos nas energias cinéti- 
cas, não para redefenir o nível de Fermi das bandas de condução dos dois metais, mas sim, para identificar posteriormente $\mu_{P}-\mu_{S}=e \phi$ como a energia potencial elétrica aplicada entre o metal hospedeiro e a ponta do microscópio. Por conveniência, trabalharemos com $\mu_{S}=0$.

Seguindo os passos de Mahan [3], introduzimos essa diferença de potencial redefinindo os Hamiltonianos como

$$
K_{o}^{A}=H_{o}^{A}
$$

e

$$
K_{o}^{P}=H_{o}^{P}-\mu_{P} \sum_{k, \sigma} c_{p k \sigma}^{+} c_{p k \sigma}
$$

onde o Hamiltoniano total será agora dado por

$$
K_{o}=K_{o}^{A}+K_{o}^{P}
$$

Podemos definir os vetores de estado de $K_{o}$ como

$$
K_{o}|n\rangle=E_{n}|n\rangle,
$$

onde $n$ é um índice de estado. Os sistemas são independentes $\left(\left[K_{o}^{A}, K_{o}^{P}\right]=0\right)$ e por isso podemos escrever um estado $|n\rangle$ como

$$
|n\rangle=|f\rangle|g\rangle
$$

sendo $|f\rangle$ e $|g\rangle$ estados do metal hospedeiro e da ponta metálica respectivamente. 
As equações de autoenergias associadas são

$$
K_{o}^{P}|g\rangle=E_{g}^{P}|g\rangle
$$

e

$$
K_{o}^{A}|f\rangle=E_{g}^{A}|f\rangle
$$

Os autovalores de $K_{o}$ são então dados por

$$
E_{n}=E_{f}^{P}+E_{g}^{A}
$$

onde $E_{f}^{P}$ e $E_{g}^{A}$ são autoenergias dos Hamiltonianos da ponta e do metal hospedeiro respectivamente.

A fórmula de Kubo derivada para a corrente de tunelamento é

$$
I=-e \frac{i}{\hbar} \int_{-\infty}^{+\infty} \theta\left(\tau-\tau^{\prime}\right) d \tau^{\prime}\left\langle\left[\frac{d}{d \tau} N_{P}(\tau), H_{t}\left(\tau^{\prime}\right)\right]\right\rangle
$$

onde

$$
N_{P}=\sum_{k \sigma} c_{p k \sigma}^{+} c_{p k \sigma}
$$

é o operador número de elétrons da ponta metálica. A média que aparece no comutador avaliado em dois instantes diferentes $\tau$ e $\tau$, é uma média termodinâmica sobre os autoestados do Hamiltoniano $K_{o}$.

O fluxo de partículas que se estabelece entre a superfície e a ponta é conseqüência da diferença de potencial aplicada e da perturbação dada pelo Hamiltoniano de tunelamento $H_{t}(\tau)$, cuja dependênica temporal é dada por 
$H_{t}(\tau)=e^{i H_{o} \tau / \hbar} H_{t} e^{-i H_{o} \tau / \hbar}=e^{i K_{o} \tau / \hbar}\left\{e^{i \mu_{P} N_{P} \tau / \hbar} H_{t} e^{-i \mu_{P} N_{P} \tau / \hbar}\right\} e^{-i K_{o} \tau / \hbar}$

Com os resultados do Apêndice A, chega-se a

$$
H_{t}(\tau)=e^{\frac{i}{\hbar} K_{o} \tau}\left(t \sum_{k \sigma} \sum_{q \delta}\left(e^{-i \vec{k} \cdot \overrightarrow{R_{2}}} c_{s k \sigma}^{+} c_{p q \delta} e^{-\frac{i}{\hbar} \mu_{P} \tau}+\text { h.c. }\right)\right) e^{-\frac{i}{\hbar} K_{o} \tau} .
$$

Os índices de spin são $\sigma$ e $\delta$. O Hamiltoniano total é $K=K_{o}+H_{t}$. Supomos $\mu_{P}=e \phi$, há um operador corrente da ponta dado por

$$
\frac{d}{d \tau} N_{P}=\frac{i}{\hbar}\left[K, N_{P}\right]=\frac{i}{\hbar}\left[H_{t}, N_{P}\right]=\frac{i}{\hbar} t \sum_{k \sigma} \sum_{q \delta}\left(c_{s k \sigma}^{+} c_{p q \delta}-h . c .\right)
$$

ou seja,

$$
\begin{array}{r}
\frac{d}{d \tau} N_{P}(\tau)=e^{i H_{o} \tau / \hbar} \frac{d}{d \tau} N_{P} e^{-i H_{o} \tau / \hbar} \\
=e^{i K_{o} \tau / \hbar}\left\{e^{i \mu_{P} N_{P} \tau / \hbar} \frac{d}{d \tau} N_{P} e^{-i \mu_{P} N_{P} \tau / \hbar}\right\} e^{-i K_{o} \tau / \hbar}
\end{array}
$$

De maneira análoga, com base no Apêndice A, obtemos

$$
\frac{d}{d \tau} N_{P}(\tau)=e^{\frac{i}{\hbar} K_{o} \tau}\left(\frac{i}{\hbar} t \sum_{k \sigma} \sum_{q \delta}\left(e^{-i \vec{k} \cdot \overrightarrow{R_{2}}} c_{s k \sigma}^{+} c_{p q \delta} e^{-\frac{i}{\hbar} \mu_{P} \tau}-\text { h.c. }\right)\right) e^{-\frac{i}{\hbar} K_{o} \tau}
$$

Agora, a observável corrente elétrica é obtida quando introduzimos as equações (3.2) e (3.3) na equação (3.1) 
$I=\frac{e}{\hbar^{2}} \int_{-\infty}^{+\infty} d \tau^{\prime} \theta\left(\tau-\tau^{\prime}\right)\left\{e^{i e \phi\left(\tau^{\prime}-\tau\right) / \hbar}\left\langle\left[A(\tau), A^{+}\left(\tau^{\prime}\right)\right]\right\rangle-e^{-i e \phi\left(\tau^{\prime}-\tau\right) / \hbar}\left\langle\left[A^{+}(\tau), A\left(\tau^{\prime}\right)\right]\right\rangle\right\}$

onde

$$
A(\tau)=t \sum_{k \sigma p \delta} e^{-i \vec{k} \cdot \vec{R}_{2}} c_{s k \sigma}^{+}(\tau) c_{p q \delta}(\tau)
$$

e a evolução temporal dos operadores de acordo com a representação das interações é

$$
\begin{aligned}
& c_{p k \sigma}(\tau)=e^{i K_{o}^{p} \tau / \hbar} c_{p k} e^{-i K_{o}^{p} \tau / \hbar} \\
& c_{s k \delta}(\tau)=e^{i K_{o}^{A} \tau / \hbar} c_{s k} e^{-i K_{o}^{A} \tau / \hbar}
\end{aligned}
$$

Vamos subentender os símbolos de soma sem índices como somas feitas sobre todos os índices presentes nas fórmulas (3.4) e (3.5), só para compactar a notação. Temos então

$$
\begin{aligned}
I= & \frac{e t^{2}}{\hbar^{2}} \sum \int_{-\infty}^{\tau} d \tau^{\prime} e^{-i\left(\overrightarrow{k_{2}}-\overrightarrow{k_{3}}\right) \cdot \overrightarrow{R_{2}}} e^{i e \phi\left(\tau^{\prime}-\tau\right) / \hbar}\left\langle\left[c_{s k_{2}}^{+}(\tau) c_{p k_{1}}(\tau), c_{p k_{4}}^{+}\left(\tau^{\prime}\right) c_{s k_{3}}\left(\tau^{\prime}\right)\right]\right\rangle+ \\
& -\frac{e t^{2}}{\hbar^{2}} \sum \int_{-\infty}^{\tau} d \tau^{\prime} e^{i\left(\overrightarrow{k_{2}}-\overrightarrow{k_{3}}\right) \cdot \overrightarrow{R_{2}}}\left\langle\left[c_{p k_{1}}^{+}(\tau) c_{s k_{2}}(\tau), c_{s k_{3}}^{+}\left(\tau^{\prime}\right) c_{p k_{4}}\left(\tau^{\prime}\right)\right]\right\rangle e^{-i e \phi\left(\tau^{\prime}-\tau\right) / \hbar}(3 .
\end{aligned}
$$

As dependências $\overrightarrow{k_{2}}$ e $\overrightarrow{k_{3}}$ nas ondas planas que aparecem em (3.4) devem-se às construções dos operadores (3.2) e (3.3). O segundo termo é na verdade o complexo conjugado do primeiro, ou seja,

$$
I=-\frac{2 e t^{2}}{\hbar^{2}} \Im \sum \int_{-\infty}^{\tau} d \tau^{\prime} e^{i\left(\overrightarrow{k_{2}}-\overrightarrow{k_{3}}\right) \cdot \overrightarrow{R_{2}}} e^{-i e \phi\left(\tau^{\prime}-\tau\right) / \hbar}\left\langle\left[c_{p k_{1}}^{+}(\tau) c_{s k_{2}}(\tau), c_{s k_{3}}^{+}\left(\tau^{\prime}\right) c_{p k_{4}}\left(\tau^{\prime}\right)\right]\right\rangle
$$


onde $\Im$ significa a parte imaginária.

O cálculo é finalizado quando usamos novamente os resultados do Apêndice A, trocando a soma discreta em $k$ por uma integração através de uma densidade de estados $\rho_{P}\left(\varepsilon_{k}^{p}\right)$ para o Hamiltoniano da ponta de prova metálica. A corrente $I$ de tunelamento torna-se portanto

$$
I=\frac{2 \pi e^{2} t^{2} V^{2}}{\hbar} Z_{A}^{-1} \phi \sum_{f f} \int d \varepsilon_{k}^{p} \rho_{P}\left(\varepsilon_{k}^{p}\right)\left|\sum_{q \sigma}\left\langle f\left|e^{i \vec{q} \cdot \overrightarrow{R_{2}}} c_{s q}\right| f^{\prime}\right\rangle\right|^{2}\left(e^{-\beta E_{f}^{A}}+e^{-\beta E_{f}^{A},}\right)\left(-\frac{\partial}{\partial \varepsilon_{k}^{p}} f\left(\varepsilon_{k}^{p}\right)\right) \times
$$

$$
\times \delta\left(E_{f}^{A}-E_{f}^{A}+\varepsilon_{k}^{p}\right)
$$

e em seguida, calcula-se a condutância $G=\frac{I}{\phi}$.

\subsection{Funções de Green no cálculo da condutância}

Retomemos nossa expressão da condutância, já assumindo uma banda de condução chata, ou seja, aquela cuja densidade de estados varia muito pouco com a energia. Façamos a densidades de estados uma constante avaliada no nível de Fermi. A condutância pode ser reescrita como

$$
G=\frac{2 \pi e^{2}}{\hbar} t^{2} \rho_{P} \int\left\{\sum_{N, M} Z^{-1}\left(e^{-\beta E_{N}}+e^{-\beta E_{M}}\right)|\langle N|O| M\rangle|^{2} \delta\left(\varepsilon+E_{N}-E_{M}\right)\right\}\left(-\frac{\partial f}{\partial \varepsilon}\right) d \varepsilon
$$

onde por conveniência 


$$
O=\sum_{k, \sigma} e^{i \vec{k} \cdot \vec{R}_{2}} c_{s k \sigma}
$$

Os índices $N$ e $M$ são índices dos autoestados do Hamiltoniano $K_{o}^{A}$.

A função de Green retardada associada ao operador $O$ é dada por

$$
G^{R}(\varepsilon)=\sum_{N, M} Z^{-1}|\langle N|O| M\rangle|^{2} \frac{\left(e^{-\beta E_{N}}+e^{-\beta E_{M}}\right)}{\varepsilon+E_{N}-E_{M}+i \delta}
$$

No espaço das energias, o termo entre chaves da fórmula (3.7) permite escrever a condutância como

$$
G=\frac{4 \pi e^{2}}{\hbar} t^{2} \rho_{P} \int\left[-\frac{1}{\pi} \Im\left\{G^{R}(\varepsilon)\right\}\right]\left(-\frac{\partial f}{\partial \varepsilon}\right) d \varepsilon,
$$

onde o fator 2 apareceu devido ao spin.

Utilzando-se de resultados do Apêndices B e escolhendo a origem em $\vec{R}_{1}$, derivamos

$$
(\varepsilon+i \delta) G^{R}(\varepsilon)=\sum_{k}+\sum_{k, q} \varepsilon_{k}^{s} e^{i(\vec{k}-\vec{q}) \cdot \vec{R}_{2}} G_{k q}^{R}(\varepsilon)+\sum_{k, q} V e^{i(\vec{k}-\vec{q}) \cdot \vec{R}_{2}} G_{d q}^{R}(\varepsilon)
$$

e com o rearranjo apropriado dos termos da equação anterior, verificamos que

$$
\begin{aligned}
(\varepsilon+i \delta) & G^{R}(\varepsilon)=\sum_{k}+\sum_{k}\left(\varepsilon_{k}^{s} G_{k k}^{R}(\varepsilon)+V G_{d k}^{R}(\varepsilon)\right) \\
+ & \sum_{k \neq q}\left(\varepsilon_{k}^{s} G_{k q}^{R}(\varepsilon)+V G_{d q}^{R}(\varepsilon)\right) e^{i(\vec{k}-\vec{q}) \cdot \vec{R}_{2}} .
\end{aligned}
$$

Vemos que a contribuição que independe da distância entre a ponta de prova e a impureza está contida nas funções de Green $G_{k k}^{R}(\varepsilon)$ e $G_{d k}^{R}(\varepsilon)$. O passo seguinte consiste em determiná-las. Resultados do Apêndice B levam a

$$
\left(\varepsilon-\varepsilon_{k}^{s}+i \delta\right) G_{k q}^{R}(\varepsilon)=\delta_{k q}+V G_{d q}^{R}(\varepsilon)
$$


No caso $\mathrm{k}=\mathrm{q}$ temos

$$
G_{k k}^{R}(\varepsilon)=\frac{1+V G_{d k}^{R}(\varepsilon)}{\varepsilon-\varepsilon_{k}^{s}+i \delta}
$$

e no caso $q \neq k$, temos

$$
G_{k q}^{R}(\varepsilon)=\frac{V G_{d q}^{R}(\varepsilon)}{\varepsilon-\varepsilon_{k}^{s}+i \delta}
$$

Uma relação final procurada é

$$
G_{d q}^{R}(\varepsilon)=\frac{V}{\varepsilon-\varepsilon_{k}^{s}+i \delta} G_{d d}^{R}(\varepsilon)
$$

Assim, ao combinarmos (3.12), (3.13), (3.14) e (3.15) em (3.11), obtemos finalmente ao fazer troca de $\overrightarrow{R_{2}}$ por $\vec{R}$ que

$$
G^{R}(\varepsilon)=\sum_{k} \frac{1}{\varepsilon-\varepsilon_{k}^{s}+i \delta}+V^{2}\left[\sum_{k} \frac{e^{i \vec{k} \cdot \vec{R}}}{\varepsilon-\varepsilon_{k}^{s}+i \delta}\right] \times\left[\sum_{q} \frac{e^{-i \vec{q} \cdot \vec{R}}}{\varepsilon-\varepsilon_{q}^{s}+i \delta}\right] G_{d d}^{R}(\varepsilon) .
$$

Essa expressão está em concordância com resultado de Schiller e outros $\{8\}$ no caso em que não há tunelamento através da impureza.

Agora fazendo a expansão da soma sobre $k$ contida em $G^{R}(\varepsilon)$, usando a identidade $\frac{1}{x+i \delta}=\wp \frac{1}{x}-i \pi \delta(x)$ para chegar a

$$
\begin{gathered}
\Pi=\left[\sum_{k} \frac{e^{i \vec{k} \cdot \vec{R}}}{\varepsilon-\varepsilon_{k}^{s}+i \delta}\right] \times\left[\sum_{q} \frac{e^{-i \vec{q} \cdot \vec{R}}}{\varepsilon-\varepsilon_{q}^{s}+i \delta}\right]=\left|\wp \sum_{k} \frac{e^{i \vec{k} \cdot \vec{R}}}{\varepsilon-\varepsilon_{k}^{s}}\right|^{2}-\left|\pi \sum_{k} e^{-i \vec{k} \cdot \vec{R}} \delta\left(\varepsilon-\varepsilon_{k}^{s}\right)\right|^{2}+ \\
-2 i \Re\left[\wp \sum_{k} \frac{e^{i \vec{k} \cdot \vec{R}}}{\varepsilon-\varepsilon_{k}^{s}}\right] \times\left[\pi \sum_{k} e^{-i \vec{k} \cdot \vec{R}} \delta\left(\varepsilon-\varepsilon_{k}^{s}\right)\right] .
\end{gathered}
$$

Utilizando-se do Apêndice C, essa expansão resulta em 


$$
\begin{gathered}
\Pi=\frac{1}{D^{2}}\left|\frac{\Phi(\epsilon)}{q_{F} R}\right|^{2}-\frac{1}{D^{2}}\left|\pi \frac{\operatorname{sen}\left[q_{F} R\left(1+\frac{1}{2} \epsilon\right)\right]}{q_{F} R}\right|^{2}+ \\
+i 2 \pi \frac{1}{D^{2}} \frac{\operatorname{sen}\left[q_{F} R\left(1+\frac{1}{2} \epsilon\right)\right]}{q_{F} R} \frac{\Phi(\epsilon)}{q_{F} R} \\
=\frac{1}{D^{2}} S(\varepsilon, R)-i \pi \frac{1}{D^{2}} Q(\varepsilon, R),
\end{gathered}
$$

onde $q_{F}$ é o momento de Fermi.

A forma explícita de $\Phi(\epsilon)$ encontra-se no Apêndice C. Portanto, concluímos que

$$
\begin{array}{r}
-\frac{1}{\pi} \Im\left\{G^{R}(\varepsilon)\right\}=\rho_{s}(\varepsilon)+\frac{V^{2}}{D^{2}} S(\varepsilon, R)\left[-\frac{1}{\pi} \Im\left\{G_{d d}^{R}(\varepsilon)\right\}\right]+ \\
\frac{V^{2}}{D^{2}} Q(\varepsilon, R)\left[\Re\left\{G_{d d}^{R}(\varepsilon)\right\}\right] .
\end{array}
$$

Levando a expressão (3.18) em (3.9) ganhamos uma forma mais conveniente para a condutância que passa a ser dada pela expressão

$$
\begin{aligned}
G=8 \pi g_{o}\left(\frac{\Delta}{D}\right) & \int\left(-\frac{\partial f}{\partial \varepsilon}\right) d \varepsilon+8 g_{o}\left(\frac{\Gamma}{D}\right) \Delta \int S(\varepsilon, R)\left[-\frac{1}{\pi} \Im\left\{G_{d d}^{R}(\varepsilon)\right\}\right]\left(-\frac{\partial f}{\partial \varepsilon}\right) d \varepsilon+ \\
& +8 g_{o}\left(\frac{\Gamma}{D}\right) \Delta \int Q(\varepsilon, R)\left[\Re\left\{G_{d d}^{R}(\varepsilon)\right\}\right]\left(-\frac{\partial f}{\partial \varepsilon}\right) d \varepsilon
\end{aligned}
$$

Usamos as definições $g_{o}=\frac{e^{2}}{h}, \Gamma=\pi V^{2} \rho_{S}\left(\varepsilon_{F}\right)$ e $\Delta=\pi t^{2} \rho_{P}\left(\varepsilon_{F}\right)$. Supomos 
também $\rho_{P}\left(\varepsilon_{F}\right) \approx \frac{1}{D}$, ou seja, a banda livre da ponta tem a mesma meia largura que a banda do metal hospedeiro. O primeiro termo da condutância é a condutância de fundo, isto é, a condutância existente na ausência da impureza. Os demais termos são as correções devido à introdução da impureza no meio metálico. 


\section{Capítulo 4}

\section{Grupo de Renormalização}

\section{Numérico}

O grupo de renormalização numérico é uma técnica de diagonalização que se apóia na substituição do contínuo de energias da banda de condução por um conjunto discreto de valores. A discretização é feita numa escala logarítmica, por causa das divergências logarítmicas que aparecem nos cálculos perturbativos das propriedades físicas à baixas temperaturas de metais com impurezas magnéticas. Com essa discretização, tais divergências podem ser controladas. Isso ficará mais evidente quando demonstrarmos que este método é um tratamento não perturbativo. Os erros são controláveis e independentes tanto do nível de energia do orbital de impureza como do parâmetro $V$.

\subsection{Discretização Logarítmica}

Neste trabalho, vamos supor que as energias cinéticas dos elétrons de condução obedecem a uma relação de dispersão linear. Vamos chamar de $D$ a meia largura da banda de conducão e medir as energias em unidades de $D$, com relação à energia de Fermi. A discretização consiste em substituir o contínuo da banda por um conjunto de níveis discretos com energias $\pm \Lambda^{-m-z}$, onde $m=0,1,2, \ldots$ O 


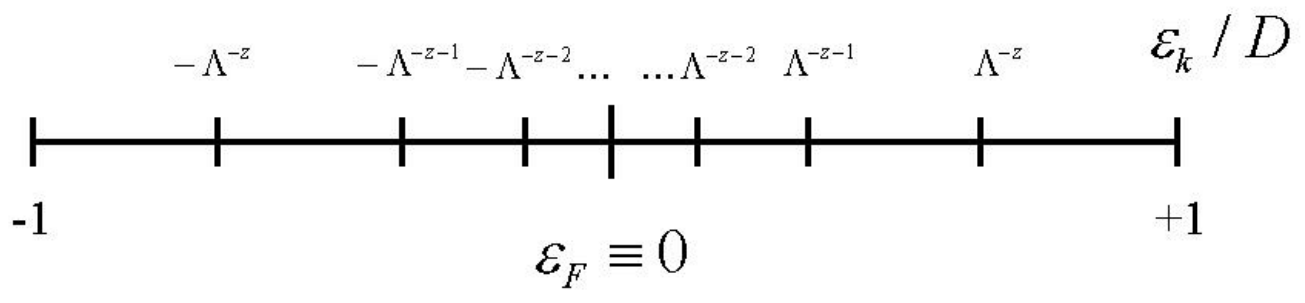

Figura 4.1: Discretização logarítmica, com $\Lambda^{-(m+z)}<|k|<\Lambda^{(1-z-m)}$ e $m=$ $1,2, \ldots, \Lambda>1$ e $0<z \leq 1$.O índice $m$ é um índice de intervalo dentro do espaco $k$ e $\Lambda$ é a razão entre dois valores consecutivos de energia. O limite $\Lambda \rightarrow 1$ recupera o espectro contínuo de energias e a discretização original [4] é obtida fazendo-se $z=1$.

espectro discreto é ilustrado na Figura 4.1

\subsection{Tridiagonalização do Hamiltoniano de Con- dução}

Ao substituirmos o contínuo por uma banda discreta, é preciso exprimir o Hamiltoniano nesta nova base. Assim, tomemos inicialmente um gás de elétrons livres, cuja energia é

$$
H_{c}=\int_{-1}^{+1} k c_{k \mu}^{+} c_{k \mu} d k
$$


Podemos definir um conjunto completo de funções de onda dentro do espaço $k$ como

$$
\Psi_{l}^{ \pm}(k)=\frac{1}{\left(1-\Lambda^{-z}\right)^{1 / 2}} e^{\left( \pm \frac{i 2 \pi l k}{1-\Lambda^{-z}}\right)},
$$

para $\Lambda^{-z}< \pm k<1$ e zero fora desse intervalo. O índice $l$ é o índice harmônico de Fourier.

Nos intervalos $n \neq 0$, define-se

$$
\Psi_{m l}^{ \pm}(k)=\frac{\Lambda^{(m+z) / 2}}{\left(1-\Lambda^{-1}\right)^{1 / 2}} e^{\left( \pm \frac{i 2 \pi \Lambda^{m+z} l k}{1-\Lambda^{-1}}\right)}
$$

para $\Lambda^{-m-z-1}<k<\Lambda^{-m-z}$ e $-\Lambda^{-m-z}<k<-\Lambda^{-m-z-1}$ e zero fora desse intervalo.

Os operadores $c_{k}$ e $c_{k}^{+}$nessa base são dados pela expansão

$$
c_{k}=\sum_{l}\left[a_{l} \Psi_{l}^{+}(k)+b_{l} \Psi_{l}^{-}(-k)\right]+\sum_{l m}\left[a_{m l} \Psi_{m l}^{+}(k)+b_{m l} \Psi_{m l}^{-}(-k)\right] .
$$

Consideraremos apenas contribuições para $l=0$, pois estamos olhando para estados centrados na impureza.

Para o intervalo $1>k>\Lambda^{-z}$, definimos o operador de Fermi normalizado

$$
a=\left(1-\Lambda^{-z}\right)^{-1 / 2} \int_{\Lambda^{-z}}^{1} c_{k} d k
$$

e no intervalo $-1<k<-\Lambda^{-z}$, temos

$$
b=\left(1-\Lambda^{-z}\right)^{-1 / 2} \int_{-1}^{-\Lambda^{-z}} c_{k} d k
$$

No caso de cada intervalo $\Lambda^{-m-z-1}<k<\Lambda^{-m-z}$ com $m=1,2, \ldots$, definimos de maneira análoga 


$$
a_{m}=\frac{\Lambda^{(m+z) / 2}}{\left(1-\Lambda^{-1}\right)^{1 / 2}} \int_{\Lambda^{-z-m-1}}^{\Lambda^{-z-m}} c_{k} d k
$$

e quando $-\Lambda^{-m-z}<k<-\Lambda^{-m-z-1}$ com $m=1,2, \ldots$, temos

$$
b_{m}=\frac{\Lambda^{(m+z) / 2}}{\left(1-\Lambda^{-1}\right)^{1 / 2}} \int_{-\Lambda^{-z-m}}^{-\Lambda^{-z-m-1}} c_{k} d k
$$

Em termos dos operadores $a, a_{m}, b$ e $b_{m}$, o Hamiltoniano da banda de condução fica

$$
H_{c}=\sum_{m=1}^{\infty} E_{m}(z, \Lambda)\left(a_{m}^{+} a_{m}-b_{m}^{+} b_{m}\right)+E_{o}(z, \Lambda)\left(a^{+} a-b^{+} b\right)
$$

onde

$$
E_{m}(z, \Lambda)=\frac{\int_{\Lambda^{-z-m}}^{\Lambda^{1-z-m}} \varepsilon_{k} d k}{\int_{\Lambda^{-z-m}}^{\Lambda^{1-z-m}} d k}=D \frac{1+\Lambda^{-1}}{2} \Lambda^{1-z-m}
$$

$$
E_{o}(z, \Lambda)=\frac{\int_{\Lambda^{-z}}^{1} \varepsilon_{k} d k}{\int_{\Lambda^{-z}}^{1} d k}=D \frac{1+\Lambda^{-z}}{2}
$$

Assim, o termo do Hamiltaniano de Anderson, que acopla a banda de condução com a impureza é

$$
H_{i c}=\sqrt{2} V\left(f_{o}^{+} c_{d}+c_{d}^{+} f_{o}\right)
$$

com

$$
f_{o}=\frac{1}{\sqrt{2}} \int_{-1}^{+1} c_{k} d k=\left[\frac{1-\Lambda^{-1}}{2}\right]^{1 / 2} \sum_{m=1}^{\infty} \Lambda^{-(z+m) / 2}\left(a_{m}+b_{m}\right)+\left[\frac{1-\Lambda^{-z}}{2}\right]^{1 / 2}(a+b) .
$$


O Hamiltoniano de condução da expressão (4.1) encontra-se escrito numa base discreta e infinita de operadores, que dificulta sua diagonalizaçao numérica. Um truncamento para um dado valor de $m$, implicaria num truncamento paralelo na série na qual o operador $f_{o}$ está expandido, comprometendo o acoplamento entre os elétrons de condução e o orbital de impureza. Faz-se necessária uma nova base de operadores que permita uma única truncagem no Hamiltoniano de Anderson.

Aplicando a construção Lanczos de vizinhos mais próximos através de um conjunto de operadores fermiônicos $f_{n}$ sobre o Hamiltoniano $H_{c}$, ganha-se uma forma tridiagonal

$$
H_{c}=\sum_{n=0}^{\infty} \varepsilon_{n}^{z}\left(f_{n}^{+} f_{n+1}+\text { h.c. }\right) .
$$

Os coeficientes $\varepsilon_{n}^{z}$, são determinados a partir do sistema de equações como mostra Yoshida e outros [5],

$$
\begin{gathered}
\prod_{n=0}^{N}\left(\varepsilon_{n}^{z}\right)^{2}=F_{N}(z, \Lambda)-\left[\left(H_{N+1}\right)^{2 N+2}\right]_{11} \\
{\left[H_{N+1}\right]_{i j}=\varepsilon_{i-1}^{z} \delta_{i, j-1}+\varepsilon_{j-1}^{z} \delta_{j, i-1}} \\
(i, j=1,2, \ldots, N+1) \\
F_{N}(z, \Lambda)=\left(1-\Lambda^{-1}\right) \sum_{m=1}^{\infty} \Lambda^{+(1-z-m)}\left[E_{m}(z, \Lambda)\right]^{2 N+2}+ \\
+\left(1-\Lambda^{-z}\right)\left[E_{o}(z, \Lambda)\right]^{2 N+2} \\
\left(\varepsilon_{o}^{z}\right)^{2}=\left(1-\Lambda^{-1}\right) \sum_{m=1}^{\infty} \Lambda^{+(1-z-m)}\left[E_{m}(z, \Lambda)\right]^{2}+ \\
+\left(1-\Lambda^{-z}\right)\left[E_{o}(z, \Lambda)\right]^{2}
\end{gathered}
$$

\subsection{Truncamento}

O truncamento pode ser feito porque os coeficientes $\varepsilon_{n}^{z}$ decaem à medida em que $\mathrm{n}$ aumenta. Verifica-se numericamente que $\varepsilon_{N}^{z}=\frac{1+\Lambda^{-1}}{2} D \Lambda^{1-z-N / 2}$ quando $N>>1$. Logo, para uma dada energia $\varepsilon=k_{B} T$, pode-se truncar o Hamilto- 
niano a um valor $\mathrm{N}$ satisfazendo a condição $\varepsilon_{N}^{z}<<\varepsilon$. Com tal truncamento, o Hamiltoniano de Anderson passa a ser, mantendo-se apenas $n<N$ :

$$
H_{\text {trunc }}=H_{i}+H_{c-i}+\sum_{n=0}^{N-1} \varepsilon_{n}^{z}\left(f_{n}^{+} f_{n+1}+\text { h.c. }\right) .
$$

Numericamente é conveniente definir um Hamiltoniano adimensional de modo que o menor autovalor seja da ordem da unidade.

Definimos então

$$
H_{N}=\frac{2}{1+\Lambda^{-1}} \Lambda^{(N-1) / 2+z-1} \frac{H_{\text {trunc }}}{D}
$$

ou seja,

$$
H_{N}=\Lambda^{(N-1) / 2}\left[\sum_{n=0}^{N-1} \xi_{n}^{z}\left(f_{n}^{+} f_{n+1}+h . c .\right)+\varepsilon_{d}^{z} c_{d}^{+} c_{d}+\sqrt{\widetilde{\Gamma}_{z}}\left(f_{o}^{+} c_{d}+h . c .\right)+U^{z} c_{d \uparrow}^{+} c_{d \uparrow} c_{d \downarrow}^{+} c_{d \downarrow}\right](4.6
$$

Aqui utilizamos as definições $\xi_{n}^{z}=\frac{2}{1+\Lambda^{-1}} \frac{\varepsilon_{n}^{z}}{D} \Lambda^{z-1}, \varepsilon_{d}^{z}=\frac{2}{1+\Lambda^{-1}} \frac{\varepsilon_{d}}{D} \Lambda^{z-1}, \sqrt{\widetilde{\Gamma}_{z}}=$ $\frac{2 \sqrt{2}}{1+\Lambda^{-1}} \frac{V}{D} \Lambda^{z-1}$ e $U^{z}=\frac{2}{1+\Lambda^{-1}} \frac{U}{D} \Lambda^{z-1}$.

O Hamiltoniano original de Anderson pode ser finalmente entendido como

$$
H_{o}^{A}=\lim _{N \rightarrow \infty} \Lambda^{-\frac{N-1}{2}} D \frac{1+\Lambda^{-1}}{2} \Lambda^{1-z} H_{N}
$$

Verificamos facilmente a seguinte relação de recorrência

$$
H_{N+1}=\Lambda^{1 / 2} H_{N}+\Lambda^{N / 2} \xi_{N}^{z} H_{N I}
$$

onde foi usado $H_{N I}=\left(f_{N, \mu}^{+} f_{N+1, \mu}+f_{N+1, \mu}^{+} f_{N, \mu}\right)$. 


\subsection{Estudo de Pontos Fixos}

Como uma ilustração da análise de pontos fixos, vamos considerar a diagonalização do Hamiltoniano da banda de condução

Vamos iniciar o estudo de pontos fixos para um caso simples, o caso de uma banda livre, isto é, $\varepsilon_{d}=0, V=0$ e $U=0$. Esse Hamiltoniano é

$$
H_{N}^{B C}=\Lambda^{(N-1) / 2} \sum_{n=0}^{N-1} \xi_{n}^{z}\left(f_{n}^{+} f_{n+1}+\text { h.c. }\right) .
$$

Uma maneira alternativa de se entender o Hamiltoniano (4.9) é escrevê-lo como

$$
H_{N}=\left(f_{o}^{+}, f_{1}^{+}, \ldots, f_{N}^{+}\right) \aleph_{N}\left(\begin{array}{c}
f_{o} \\
f_{1} \\
\vdots \\
f_{N}
\end{array}\right)
$$

onde

$$
\aleph_{N}=\Lambda^{(N-1) / 2}\left[\begin{array}{cccccccc}
0 & \xi_{0}^{z} & 0 & 0 & 0 & \cdots & 0 & 0 \\
\xi_{0}^{z} & 0 & \xi_{1}^{z} & 0 & 0 & \cdots & 0 & 0 \\
0 & \xi_{1}^{z} & 0 & \xi_{2}^{z} & 0 & \cdots & 0 & 0 \\
0 & 0 & \xi_{2}^{z} & 0 & \xi_{3}^{z} & \cdots & 0 & 0 \\
0 & 0 & 0 & \xi_{3}^{z} & 0 & \cdots & 0 & 0 \\
\vdots & \vdots & \vdots & \vdots & \vdots & \ddots & \vdots & \vdots \\
0 & 0 & 0 & 0 & 0 & \cdots & 0 & \xi_{N-1}^{z} \\
0 & 0 & 0 & 0 & 0 & \cdots & \xi_{N-1}^{z} & 0
\end{array}\right]
$$

A matriz $\aleph_{N}$ é simétrica e de ordem $N+1$. A diagonalização de $\aleph_{N}$ é feita por uma matriz M tal que $M^{-1} \aleph_{N} M$ seja uma matriz diagonal com $N+1$ autovalores 


\begin{tabular}{|c|c|c|c|c|c|c|}
\hline Auto valor & $\mathrm{z}=1, \mathrm{~N}=15$ & $\mathrm{~N}=17$ & $\mathrm{~N}=25$ & $\mathrm{z}=0.5, \mathrm{~N}=15$ & $\mathrm{~N}=17$ & $\mathrm{~N}=25$ \\
\hline \hline$\kappa_{1}$ & 0,8000483 & 0,8000479 & 0,8000477 & 0,8000429 & 0,8000461 & 0,8000477 \\
\hline$\kappa_{2}$ & 2,997492 & 2,997489 & 2,997487 & 2,997451 & 2,997475 & 2,997487 \\
\hline$\kappa_{3}$ & 9,000034 & 9,000013 & 9,000000 & 8,999716 & 8,999891 & 8,999998 \\
\hline$\kappa_{4}$ & 27,00001 & 27,00010 & 27,00000 & 26,99994 & 26,99915 & 26,99999 \\
\hline$\kappa_{5}$ & 81,00000 & 81,00002 & 81,00001 & 81,00000 & 80,99983 & 80,99989 \\
\hline$\kappa_{6}$ & 243,0000 & 243,0000 & 243,0001 & 243,0000 & 243,0000 & 242,9990 \\
\hline$\kappa_{7}$ & 729,0000 & 729,0000 & 729,0011 & 729,0000 & 729,0000 & 728,9912 \\
\hline$\kappa_{8}$ & 2187,000 & 2187,000 & 2187,008 & 1493,749 & 2187,000 & 2186,931 \\
\hline$\kappa_{9}$ & & 6561,000 & 6561,002 & & 4481,246 & 6560,986 \\
\hline$\kappa_{10}$ & & & 19683,00 & & & 19683,00 \\
\hline$\kappa_{11}$ & & & 59049,00 & & & 59049,00 \\
\hline$\kappa_{12}$ & & & 177147,0 & & & 177147,0 \\
\hline$\kappa_{13}$ & & & 531441,0 & & & 362981,0 \\
\hline
\end{tabular}

Tabela 4.1: Autovalores de energia para N ímpar.

$\kappa_{j}$. O espectro de autovalores é simétrico, ou seja, para um dado autovetor $\kappa_{j}$ existe um outro $-\kappa_{j}$.

A relação de recorrência obtida na seção 4.3 define a Transformação do Grupo de Renormalização Numérico, que será denotada por

$$
\top\left[H_{N}^{B C}\right]=H_{N+1}^{B C}
$$

Essa operação afirma que uma vez conhecidos os autovalores de $H_{N}^{B C}$ e os

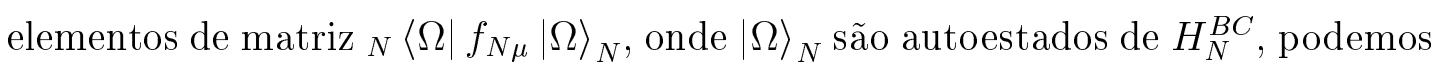
obter os autovalores de $H_{N+1}^{B C}$ e os elementos de matriz ${ }_{N+1}\left\langle\Omega\left|f_{N+1, \mu}\right| \Omega\right\rangle_{N+1}$, onde $|\Omega\rangle_{N+1}$ são autoestados de $H_{N+1}^{B C}$. Quando $\top$ opera num Hamiltoniano H e resulta o mesmo $\mathrm{H}(\mathrm{\top}[H]=H)$, dizemos que $\mathrm{H}$ é ponto fixo de $\mathrm{\top}$.

A transformação $\top$ não possui pontos fixos, mas $\top^{2}$ sim, ou seja, $\top^{2}\left[H_{N}^{B C}\right]=$ $H_{N+2}^{B C}$. Essa operação é também uma tranformação do GRN. Essa propriedade pode ser observada a partir do espectro de energias de $H_{N}^{B C}$ antes e depois da transformação $T^{2}$, em que o segundo conjunto de energias difere do primeiro pelo aparecimento de um novo autovalor acrescentado após a operação. Nas tabelas 4.1 e 4.2 apresentamos algumas diagonalizações com $\Lambda=3$. 


\begin{tabular}{|c|c|c|c|c|}
\hline Auto Valor & $\mathrm{z}=1, \mathrm{~N}=16$ & $\mathrm{~N}=18$ & $\mathrm{z}=0.5, \mathrm{~N}=16$ & $\mathrm{~N}=18$ \\
\hline \hline$\kappa_{1}$ & 1,695755 & 1,695755 & 1,695755 & 1,695751 \\
\hline$\kappa_{2}$ & 5,196104 & 5,196099 & 5,196034 & 5,196075 \\
\hline$\kappa_{3}$ & 15,58852 & 15,58848 & 15,58797 & 15,58827 \\
\hline$\kappa_{4}$ & 46,76538 & 46,76555 & 46,76527 & 46,76390 \\
\hline$\kappa_{5}$ & 140,296 & 140,2962 & 140,2961 & 140,2958 \\
\hline$\kappa_{6}$ & 420,888 & 420,8884 & 420,8883 & 420,8883 \\
\hline$\kappa_{7}$ & 1262,66 & 1262,665 & 1262,665 & 1262,665 \\
\hline$\kappa_{8}$ & 3787,99 & 3787,995 & 2587,249 & 3787,995 \\
\hline$\kappa_{9}$ & & 11363,99 & & 7761,746 \\
\hline
\end{tabular}

Tabela 4.2: Autovalores de energia para N par

Vêmos para $N$ ímpar e $N>>1$ que os autovalores são aproximadamente dados por

$$
\kappa_{j}=\Lambda^{j-1}, \quad j=1, \ldots,(N-1) / 2
$$

e

$$
\kappa_{(N+1) / 2}=\frac{1+\Lambda^{-z}}{1+\Lambda^{-1}} \Lambda^{(N-1) / 2+z-1}
$$

para $j=(N+1) / 2$.

Vemos para o caso de $N$ par e $N>>1$ que os autovalores podem ser dados por

$$
\kappa_{j}=\Lambda^{j-1 / 2}, \quad j=1, \ldots,(N-2) / 2
$$

e

$$
\kappa_{N / 2}=\frac{1+\Lambda^{-z}}{1+\Lambda^{-1}} \Lambda^{(N-1) / 2+z-1}
$$

$\operatorname{para} j=N / 2$.

A recuperação da escala física de energia é obtida, fazendo-se 


$$
\epsilon_{j}=\frac{1+\Lambda^{-1}}{2} \Lambda^{-(N-1) / 2+1-z} D \kappa_{j}
$$

Os casos em que $U=0$ e $U \neq 0$ serão discutidos detalhadamente nos próximos capítulos. 


\section{Capítulo 5}

\section{Modelo do Nível Ressonante}

O modelo do Nível Ressonante é o Hamiltoniano de Anderson com $U=0$ :

$$
H_{o}^{A}=\sum_{k \sigma} \varepsilon_{k}^{s} c_{s k \sigma}^{+} c_{s k \sigma}+\sum \varepsilon_{d} c_{d \sigma}^{+} c_{d \sigma}+V \sum_{k \sigma}\left(c_{s k \sigma}^{+} c_{d \sigma}+h . c .\right) .
$$

Fisicamente, para $\varepsilon_{d}<0$, o nível de impureza tende a ficar duplamente ocupado. O Hamiltoniano do Modelo pode ser diagonalizado exatamente e suas propriedades físicas obtidas analiticamente.

Outra quantidade importante dentro do Modelo do Nível Ressonante, é a densidade espectral de impureza. A expressão exata dessa quantidade, válida para $\left|\varepsilon_{d}\right|<<D$ é

$$
\rho_{d}(\epsilon)=\frac{1}{\pi} \frac{\Gamma}{\left(\epsilon-\varepsilon_{d}\right)^{2}+\Gamma^{2}},
$$

uma Lorentziana centrada em $\varepsilon_{d}$ e com meia largura $\Gamma$. A interpretação física é imediata, o acoplamento com a banda de condução alarga o nível $\varepsilon_{d}$.

O objetivo desse capítulo é apresentar inicialmente uma abordagem analítica do problema na derivação de uma fórmula exata da condutância. Depois, utilizaremos o Grupo de Renormalização Numérico na obtenção de uma fórmula que depende da diagonalização numérica do Hamiltoniano de Anderson. Dados esses 


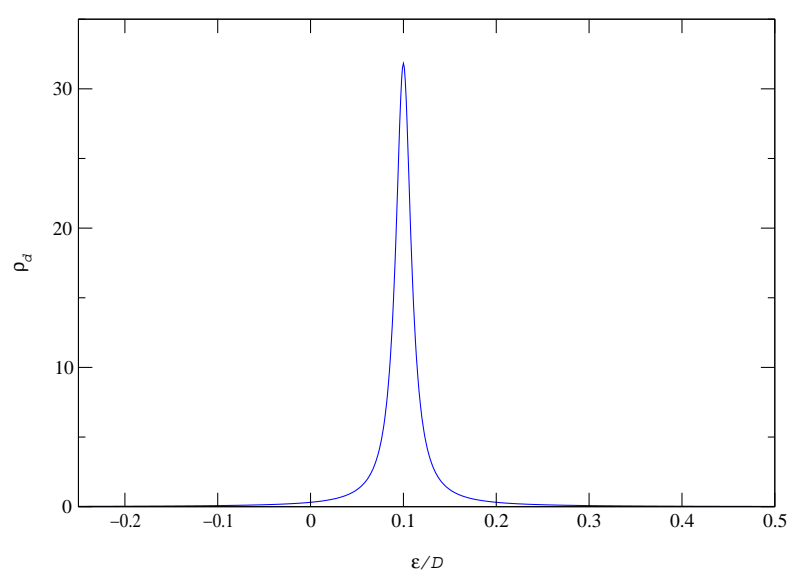

Figura 5.1: Densidade Espectral de Impureza contra energia com $\varepsilon_{d}=0,1 D$ e $\Gamma=0,01 D$.

dois passos, mostraremos os resultados gerados pelos dois métodos acompanhados de suas respectivas interpretações físicas. Mostraremos, também, o excelente desempenho do Grupo de Renormalização Numérico no cálculo de propriedades termodinâmicas através da perfeita concordância com os resultados analíticos.

\subsection{Fórmula exata da condutância}

No caso $U=0$ a função de Green retardada associada à impureza de acordo com [7] é

$$
G_{d d}^{R}(\varepsilon)=\left(\varepsilon-\varepsilon_{d}+i \delta-V^{2} \sum_{k} \frac{1}{\varepsilon-\varepsilon_{k}^{s}+i \delta}\right)^{-1}
$$

Fazendo as introduções das definições $\varepsilon=\lambda D, \varepsilon_{d}=\epsilon_{d} D, \Theta=\frac{k_{B} T}{D}$ e usando o resultado do Apêndice B, ganhamos uma fórmula final para a condutância que é 


$$
\begin{aligned}
G=g_{o} 8 & \pi\left(\frac{\Delta}{D}\right) \frac{\operatorname{senh}\left(\frac{1}{\Theta}\right)}{1+\cosh \left(\frac{1}{\Theta}\right)}+g_{o} 8\left(\frac{\Gamma}{D}\right)\left(\frac{\Delta}{D}\right) \int d \lambda S(\lambda, R) \rho_{d}(\lambda)\left(-\frac{\partial f}{\partial \lambda}\right)+ \\
& +g_{o} 8\left(\frac{\Gamma}{D}\right)\left(\frac{\Delta}{D}\right) \int d \lambda Q(\lambda, R) \frac{1}{\pi} \frac{\left(\lambda-\epsilon_{d}\right)}{\left(\frac{\Gamma}{D}\right)^{2}+\left(\lambda-\epsilon_{d}\right)^{2}}\left(-\frac{\partial f}{\partial \lambda}\right)
\end{aligned}
$$

onde

$$
\rho_{d}(\lambda)=\frac{\frac{\Gamma}{D}}{\left(\frac{\Gamma}{D}\right)^{2}+\left(\lambda-\epsilon_{d}\right)^{2}}
$$

e

$$
\left(-\frac{\partial f}{\partial \lambda}\right)=\frac{1}{\Theta} \frac{e^{-\frac{\lambda}{\Theta}}}{\left(1+e^{-\frac{\lambda}{\Theta}}\right)^{2}}
$$

Nosso interesse é o de estudar as mudanças que o segundo e o terceiro termos, presentes devido à introdução da impureza no meio metálico, provocam na condutância de fundo (primeiro termo). Assim, é conveniente definir a quantidade $\frac{\Delta G}{G_{o}}=\frac{G-G_{o}}{G_{o}}$ como medidor dessa informação e analisar o seu comportamento em diversos casos . Foi definido $G_{o}$ para se referir à condutância de fundo.

Na seção 5.3, será mostrada através de estudos mais detalhados que a contribuição da parte real da função de Green de impureza é uma quantidade desprezível principalmente à baixas temperaturas.

\subsection{Fórmula da Condutância via GRN}

O Hamiltoniano do Modelo do Nível Ressonante, já adimensional e escrito na base dos $f_{n}$ é 


$$
H_{N}=\Lambda^{(N-1) / 2}\left[\sum_{n=0}^{N-1} \xi_{n}^{z}\left(f_{n}^{+} f_{n+1}+h . c .\right)+\varepsilon_{d}^{z} c_{d}^{+} c_{d}+\sqrt{\widetilde{\Gamma}_{z}}\left(f_{o}^{+} c_{d}+h . c .\right)\right]
$$

que pode ser escrito na forma matricial

$$
H_{N}=\left(c_{d}^{+}, f_{o}^{+}, f_{1}^{+}, \ldots, f_{N}^{+}\right) \aleph_{N}\left(\begin{array}{c}
c_{d} \\
f_{o} \\
f_{1} \\
\vdots \\
f_{N}
\end{array}\right)
$$

onde

$$
\aleph_{N}=\Lambda^{(N-1) / 2}\left[\begin{array}{cccccccc}
\varepsilon_{d}^{z} & \sqrt{\widetilde{\Gamma}_{z}} & 0 & 0 & 0 & \cdots & 0 & 0 \\
\sqrt{\widetilde{\Gamma}_{z}} & 0 & \xi_{0}^{z} & 0 & 0 & \cdots & 0 & 0 \\
0 & \xi_{0}^{z} & 0 & \xi_{1}^{z} & 0 & \cdots & 0 & 0 \\
0 & 0 & \xi_{1}^{z} & 0 & \xi_{2}^{z} & \cdots & 0 & 0 \\
0 & 0 & 0 & \xi_{2}^{z} & 0 & \cdots & 0 & 0 \\
\vdots & \vdots & \vdots & \vdots & \vdots & \ddots & \vdots & \vdots \\
0 & 0 & 0 & 0 & 0 & \cdots & 0 & \xi_{N-1}^{z} \\
0 & 0 & 0 & 0 & 0 & \cdots & \xi_{N-1}^{z} & 0
\end{array}\right] .
$$

Assim, a base de operadores diagonais é dada pela transformação

$$
\left(\begin{array}{c}
g_{1} \\
\vdots \\
g_{j} \\
\vdots \\
g_{N+2}
\end{array}\right)=M^{-1}\left(\begin{array}{c}
c_{d} \\
\vdots \\
f_{n} \\
\vdots \\
f_{N}
\end{array}\right)
$$

onde $\mathrm{M}$ é a matriz que diagonaliza $\aleph_{N}$. Alternativamente podemos escrever 


$$
g_{j}=u_{j d} c_{d}+\sum_{n=0}^{N} u_{j n} f_{n}
$$

onde $u_{j d}$ são os elementos da matriz M.

Ficará evidente mais adiante, que no cálculo numérico da condutância através do grupo de renormalização, é conveniente usar a expansão do operador $c_{d}$ na base de operadores diagonais $g_{j}$. Essa expansão é

$$
c_{d}=\sum_{j} u_{j d} g_{j}
$$

A Figura 5.2 mostra um estudo dos autovalores, em que ilustramos o comportamento do segundo autovalor positivo. O ponto azul é para $N$ par e o ponto vermelho é para $N$ ímpar. Como se vê para cada paridade de $N$, existe um comportamento bastante regular.

Lembrando da expressão geral da condutância, vamos aqui negligenciar a parte real da função de Green associada à impureza, isto é,

$$
G=8 \pi g_{o}\left(\frac{\Delta}{D}\right) \int\left(-\frac{\partial f}{\partial \varepsilon}\right) d \varepsilon+8 g_{o}\left(\frac{\Gamma}{D}\right) \Delta \int S(\varepsilon, R)\left[-\frac{1}{\pi} \Im\left\{G_{d d}^{R}(\varepsilon)\right\}\right]\left(-\frac{\partial f}{\partial \varepsilon}\right) d \varepsilon
$$

Esta seção usará a função de Green associada à impureza na forma

$$
G_{d d}^{R}(\varepsilon)=\sum_{N, M} Z^{-1}\left|\left\langle N\left|c_{d}\right| M\right\rangle\right|^{2} \frac{\left(e^{-\beta E_{N}}+e^{-\beta E_{M}}\right)}{\varepsilon+E_{N}-E_{M}+i \delta}
$$

de onde segue

$$
-\frac{1}{\pi} \Im\left\{G_{d d}^{R}(\varepsilon)\right\}=\sum_{N, M} Z^{-1}\left|\left\langle N\left|c_{d}\right| M\right\rangle\right|^{2}\left(e^{-\beta E_{N}}+e^{-\beta E_{M}}\right) \delta\left(\varepsilon+E_{N}-E_{M}\right)
$$




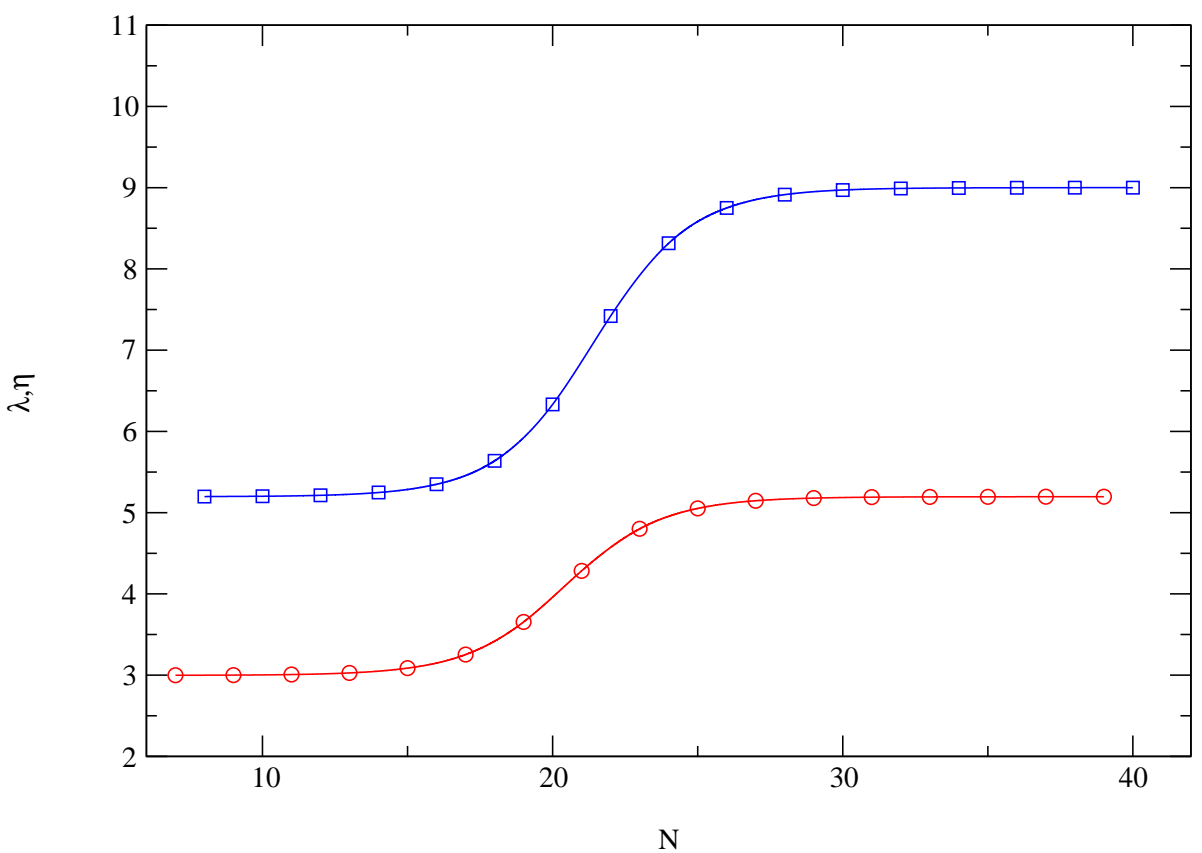

Figura 5.2: Evolução de um autovalor com N. A curva vermelha se refere ao segundo autovalor para $\mathrm{N}$ ímpar $(\lambda)$ e a curva azul para $\mathrm{N}$ par $(\eta)$. Nas proximidades de $N=20$ o autovalor $\lambda$ ( $N$ ímpar) se comporta como ponto fixo de $N$ par. Os parâmetros usados foram $\Lambda=3, V=0,05 D$ e $\varepsilon_{d}=0$. 


$$
\Re\left\{G_{d d}^{R}(\varepsilon)\right\}=\wp \sum_{N, M} Z^{-1}\left|\left\langle N\left|c_{d}\right| M\right\rangle\right|^{2} \frac{\left(e^{-\beta E_{N}}+e^{-\beta E_{M}}\right)}{\varepsilon+E_{N}-E_{M}} .
$$

Focalizando a atenção na parte imaginária da função de Green, é possível escrever

$$
-\left.\frac{\partial}{\partial \varepsilon} f\right|_{\varepsilon=E_{M}-E_{N}}=\frac{e^{\beta\left(E_{M}-E_{N}\right)}}{\left[1+e^{\beta\left(E_{M}-E_{N}\right)}\right]^{2}} \beta
$$

Assim, chegamos a

$$
-\frac{1}{\pi} \Im\left\{G_{d d}^{R}(\varepsilon)\right\}\left(-\frac{\partial f}{\partial \varepsilon}\right)=\beta \sum_{N, M} Z^{-1} \frac{\left|\left\langle N\left|c_{d}\right| M\right\rangle\right|^{2}}{e^{+\beta E_{N}+e^{+\beta E_{M}}}} \delta\left(\varepsilon+E_{N}-E_{M}\right) .
$$

Nesse momento, percebemos a utilidade da expansão do operador $c_{d}$ na base de operadores diagonais $g_{j}$ (expressão (5.7)).

O cálculo do elemento de matriz $\left\langle N\left|c_{d}\right| M\right\rangle$ nos informa que os estados $|N\rangle$ e $|M\rangle$ diferem apenas por um elétron, isto é, $E_{M}-E_{N}=\epsilon_{j}=\frac{1+\Lambda^{-1}}{2} \Lambda^{-(N-1) / 2+1-z} D \lambda_{j}$, onde $\lambda_{j}$ é um autovalor calculado numericamente.

Assim, a condutância, desprezada a contribuição da parte real de $G_{d d}^{R}(\varepsilon)$ é

$$
G(z)=g_{o} 8 \pi\left(\frac{\Delta}{D}\right) \frac{\operatorname{senh}\left(\frac{1}{\Theta}\right)}{1+\cosh \left(\frac{1}{\Theta}\right)}+g_{o} 8\left(\frac{\Gamma}{D}\right)\left(\frac{\Delta}{D}\right) \sum_{j}\left|S\left(\lambda_{j}, R\right)\right|^{2} u_{j d}^{2}\left(-\frac{\partial f}{\partial \lambda}\right)_{\lambda=\lambda_{j}} .
$$

A fórmula (5.8) de condutância acima não corresponde à condutância verdadeira, uma vez que o espectro de autovalores empregado está definido numa base discreta. Calculadas nessa base, propriedades termodinâmicas como suscetibilidade magnética ou a condutância oscilam com a temperatura. Esse ponto será abordado na próxima subseção. É necessário recuperarmos a condutância na base contínua. Isso pode ser feito através da generalização do Grupo de Renormalização Numérico para o cálculo de propriedades termodinâmicas in- 
troduzida por Oliveira e outros [6]. Em nosso problema, já é suficiente considerarmos a média da condutância entre dois valores de $z$; por exemplo $z=0,5$ e $z=1,0$, isto é

$$
G=\frac{G(z=0,5)+G(z=1,0)}{2} .
$$

Nesta dissertação, em todos os casos a condutância foi obtida utilizando-se $z=0,5$ e $z=1,0$.

\subsection{Resultados}

Nesta seção, apresentamos resultados do cálculo da contutância obtidos a partir das expressões exata e numérica (GRN) para diferentes valores dos parâmetros do modelo.

Inicialmente, vamos analisar a expressão exata da condutância em função da temperatura para uma distância fixa da ponta de prova. Essa análise visa comparar a expressão completa, aquela que possui as partes imaginária e real da função de Green de impureza, com a incompleta, que despreza a contribuição da parte real dessa função de Green.

Na Figura 5.3, o objetivo é mostrar o grau de influência da parte real da função de Green de impureza na condutância em função da temperatura através da comparação das expressões completa e incompleta. A motivação desse estudo ficará mais clara quando abordarmos o caso interagente (capítulo 6), no qual a determinação numérica da parte real da função de Green de impureza é mais complicada.

A Figura 5.3 confirma o antecipado na seção 5.1, onde já foi mencionada a pequena contribuição da parte real da função de Green no regime de baixas energias. Vê-se que existe uma discrepância desprezível a energias próximas à meia largura de banda. Pode-se concluir que considerar unicamente a parte imaginária 


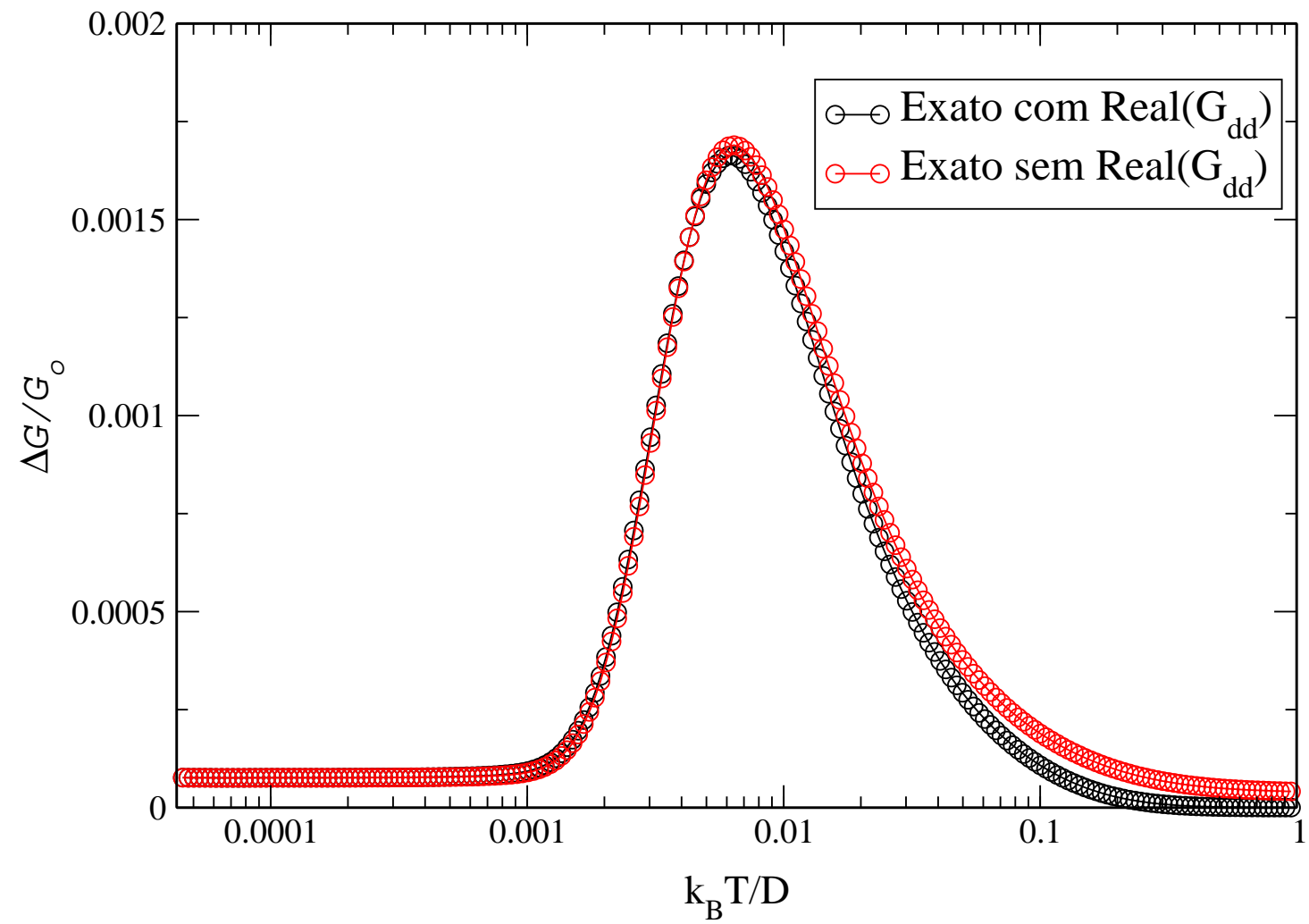

Figura 5.3: O gráfico compara a curva gerada considerando a parte real da função de Green associada à impureza $G_{d d}(\varepsilon)$ (preta) com aquela desconsiderando a parte real (vermelha). Os parâmetros utilizados foram $V=t=0,01 D, \varepsilon_{d}=$ $-0,01 D$ e $q_{F} R=\pi$. Esse resultado confirma a pequena influência da parte real da função de Green na condutância conforme, adiantado na seção 5.1 
da função de Green no cálculo da condutância em função da temperatura gera um erro desprezível na faixa de energias de interesse, o regime de baixas temperaturas. A escolha dos parâmetros do modelo não afetam essa conclusão. O resultado da Figura 5.3 mostra também que os estados relevantes do Hamiltoniano de Anderson que participam do tunelamento são aqueles que diferem em energia por um único elétron que sai ou chega na ponta de prova, ou seja, são aqueles estados cuja diferença de energia ressoa com a energia de uma partícula da ponta, contidos na parte imaginária da função de Green.

A explicação da forma da curva fica ditada essencialmente pela densidade espectral de impureza e a derivada de função da Fermi presentes na fórmula da condutância, para um conjunto fixo de parâmetros do modelo.

Vamos analisar as três regiões de importância na Figura 5.3, como o valor constante da condutância a baixas temperaturas, o valor máximo em temperaturas intermediárias e a diminuição da condutância a altas temperaturas.

A derivada da função de Fermi é uma função cuja largura é da ordem de $k_{B} T$ e por isso é ela quem determina as energias eletrônicas envolvidas no tunelamento. Isso significa para ela, que somente os estados em torno da energia térmica $k_{B} T$ são os mais importantes no cálculo da condutância. No limite de baixas temperaturas, a derivada da função de Fermi é praticamente uma distribuição Delta de Dirac, e por isso nas temperaturas próximas de zero somente os elétrons nas vizinhanças do nível de Fermi estão envolvidos no tunelamento. Assim, podemos entender o patamar na condutância nesse intervalo de temperatura, onde a condutância deixa de ser uma função da temperatura e passa a ser uma constante.

A Figura 5.3 também mostra que existe uma região em que a condutância aumenta com o aumento da temperatura e depois diminui com o aumento da temperatura até que se aproxime do valor zero. Existe assim, uma região onde a condutância passa por um máximo.

A condutância é basicamente a soma sobre as energias da banda de condução da ponta por meio do produto da função densidade espectral de impureza pela 
derivada da distribuição de Fermi. A temperatura governa apenas a distribuição de Fermi, pois à medida em que a temperatura aumenta, a derivada da função de Fermi deixa de ser mais aguda no nível de Fermi e menos estreita em sua largura. A densidedade espectral de impureza, diferentemente, é praticamente constante em função da temperatura. A forma da condutância, fixados os parâmetros é governada portanto, pela derivada da função de Fermi.

Nessa soma em energia é possível concluir que existe uma competição entre os elétrons térmicos, aqueles com energias dentro da faixa de energia $k_{B} T$ ditados pela derivada da distribuição de Fermi, e aqueles cujas energias ressonam com nível de energia $\varepsilon_{d}$ do orbital de impureza, ditados pela densidade espectral de impureza. A derivada da função de Fermi alarga com a temperatura.

É sabido que o aumento da temperatura deixa menos intensa a ressonância no nível de Fermi e aumenta a largura da derivada da função de Fermi. A elevação da temperatura transforma a função distribuição de Fermi numa distribuição de Boltzman, cuja forma é chata e larga para temperaturas elevadas. Isso explica o decaimento da condutância. A função de Fermi comanda os extremos de energia térmica, enquanto que para valores nem tão baixos e nem tão altos de temperaturas, existe uma competição entre a densidade espectral de impureza e a derivada da distribuição de Fermi. Assim, podemos explicar o pico na condutância, que se deve essecialmente a contribuição da densidade espectral de impureza.

O segundo passo desta seção é confrontar o resultado exato obtido na Figura 5.3 com o gerado pelo Grupo de Renormalização Numérico. Antes disso, é preciso retomar o assunto sobre as oscilações das propriedades termodinâmicas devido a discretização da banda de condução introduzida pelo método. A Figura 5.4 mostra três curvas de condutância geradas pela expressão numérica, duas curvas com dois valores distintos de z e uma última curva obtida pela média das duas anteriores. Foi escolhida uma região de baixas temperaturas, na qual, essas oscilações são mais evidentes.

A Figura 5.4 mostra para eliminar as oscilações da condutância, basta uma 


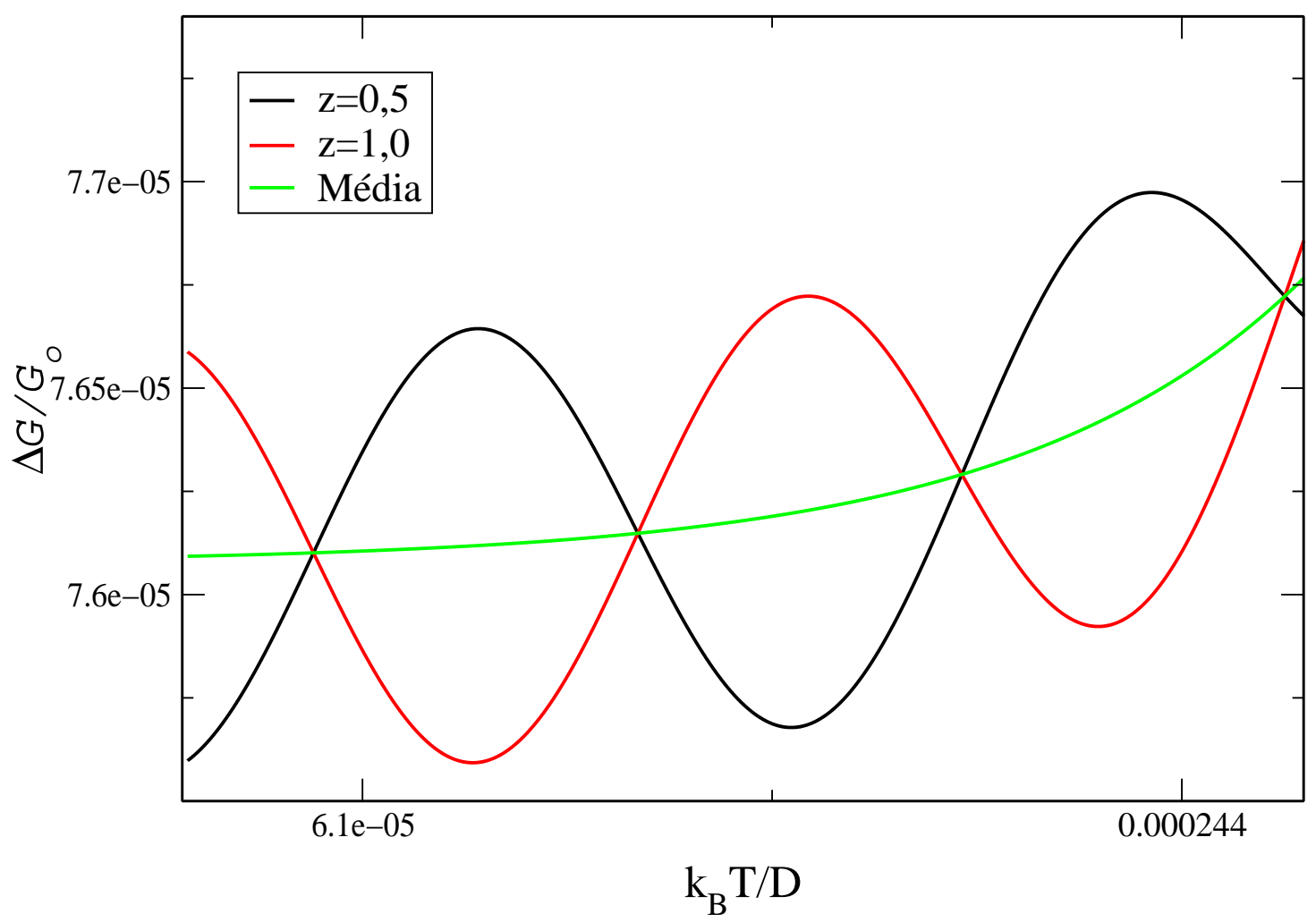

Figura 5.4: Oscilações da condutância a baixas temperaturas nos casos $\mathrm{z}=0,5$ (curva preta) e $\mathrm{z}=1,0$ (curva vermelha). A condutância verdadeira (curva verde) é a média entre as condutâncias com $z=0,5$ e com $z=1,0$. Os parâmetros utilizados foram $N=25, \Lambda=3, V=t=0,01 D, \varepsilon_{d}=-0,01 D$ e $q_{F} R=\pi$. 
média entre duas condutâncias calculadas em dois valores de $\mathrm{z}$, no caso $z=0,5$ e $z=1,0$. Na curva verde, a condutância fica suavizada com esse procedimento. O mesmo cálculo para $\Lambda=2$ geraria curvas preta e vermelha com oscilações de amplitudes menor, mas com a média indistingüível da curva verde da Figura 5.4. Verifica-se assim que a média da condutância converge rapidamente para o contínuo quando $\Lambda \rightarrow 1$. É dessa forma que eliminamos a inconveniência das oscilações na determinação das propriedades termodinâmicas.

A Figura 5.5 foi gerada para valores de temperatura mais altos do que o intervalo de temperatura da Figura 5.4 e mostra a comparação entre o resultado exato e numérico (GRN) da condutância contra a temperatura. A parte real da função de Green de impureza pouco afeta o cálculo da condutância em função da temperatura como aprendemos na Figura 5.3. Assim sendo, precisamos comparar o cálculo exato desconsiderando a parte real da função de Green com o cálculo numérico (GRN) que também desconsidera essa parte. A Figura 5.5 mostra essa comparação.

O resultado exibido pela Figura 5.5 revela que os erros introduzidos pelo método do Grupo de Renormalização Numérico são desprezíveis, ou seja, a utilização de algumas energias calculadas a partir da diagonalização do Hamiltoniano de Anderson escrito numa base discreta e finita consegue substituir sem perda de precisão a soma contínua da condutância exata. O resultado da Figura 5.5 mostra a eficiência do método.

O terceiro passo consiste em alterar os parâmetros anteriormente empregados. Vamos alterar apenas o valor do nível de energia do orbital de impureza e analisar fisicamente o resultado obtido.

No caso $\varepsilon_{d}=0$ (curva azul), temos no lugar de um pico da condutância a temperaturas intermediárias, um patamar bem mais elevado a baixas temperaturas. Esse efeito a baixas temperaturas deve-se simultaneamente à derivada da função de Fermi, que torna relevantes no processo de tunelamento somente os elétrons próximos do nível de Fermi, e também principalmente à densidade espectral de 46 


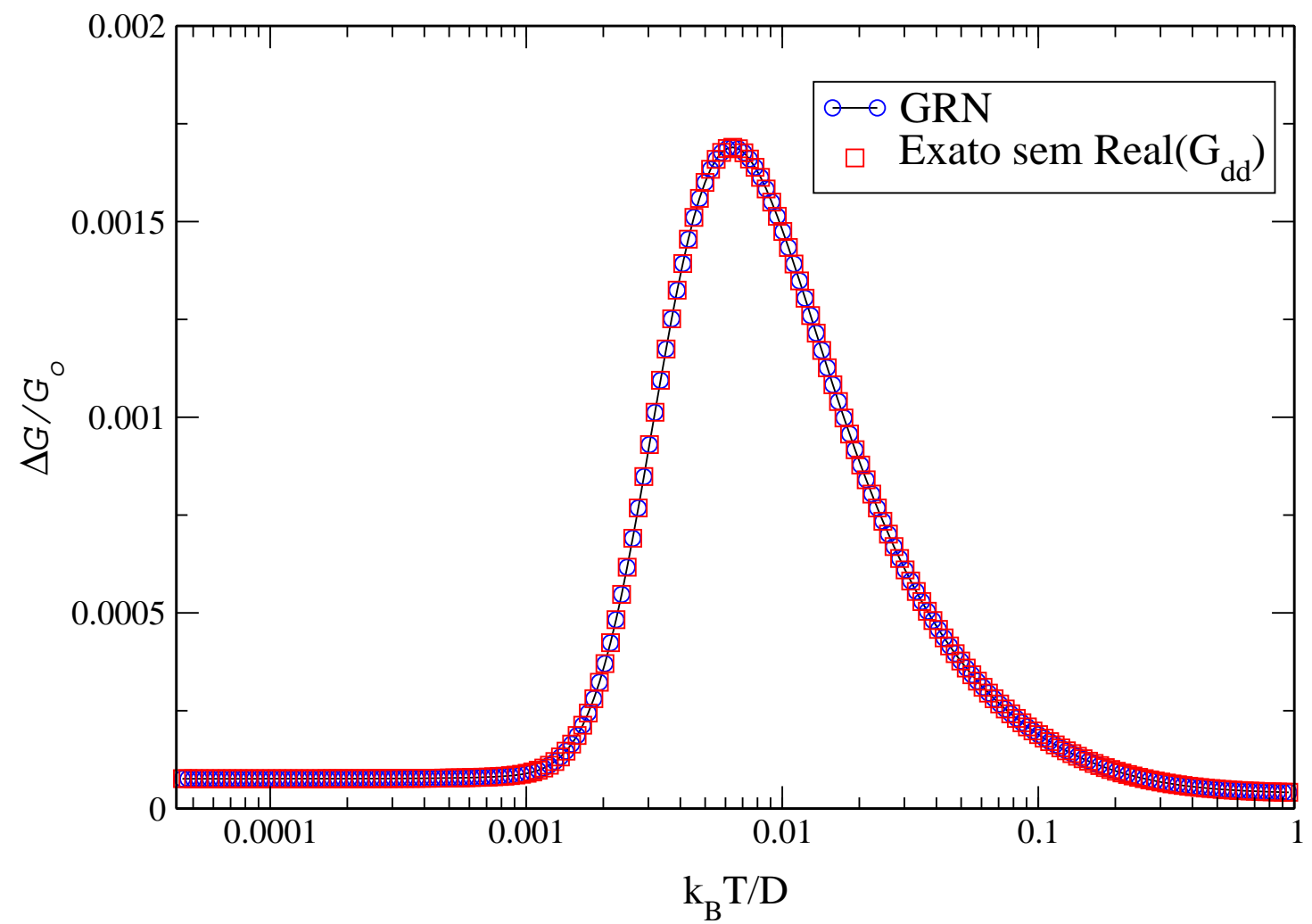

Figura 5.5: O gráfico compara a curva azul gerada pelo Grupo de Renormalização Numérico ( $N=25$ e $\Lambda=3$ ) com a fórmula exata (curva vermelha) usando $V=t=0,01 D, \varepsilon_{d}=-0,01 D$ e $q_{F} R=\pi$. Em ambos os casos, $\Re\left\{G_{d d}(\varepsilon)\right\}$ foi negligenciada. Esse gráfico mostra que a generalização da técnica de Grupo de Renormalização para cálculo de propriedades termodinâmicas produz resultados excelentes. 


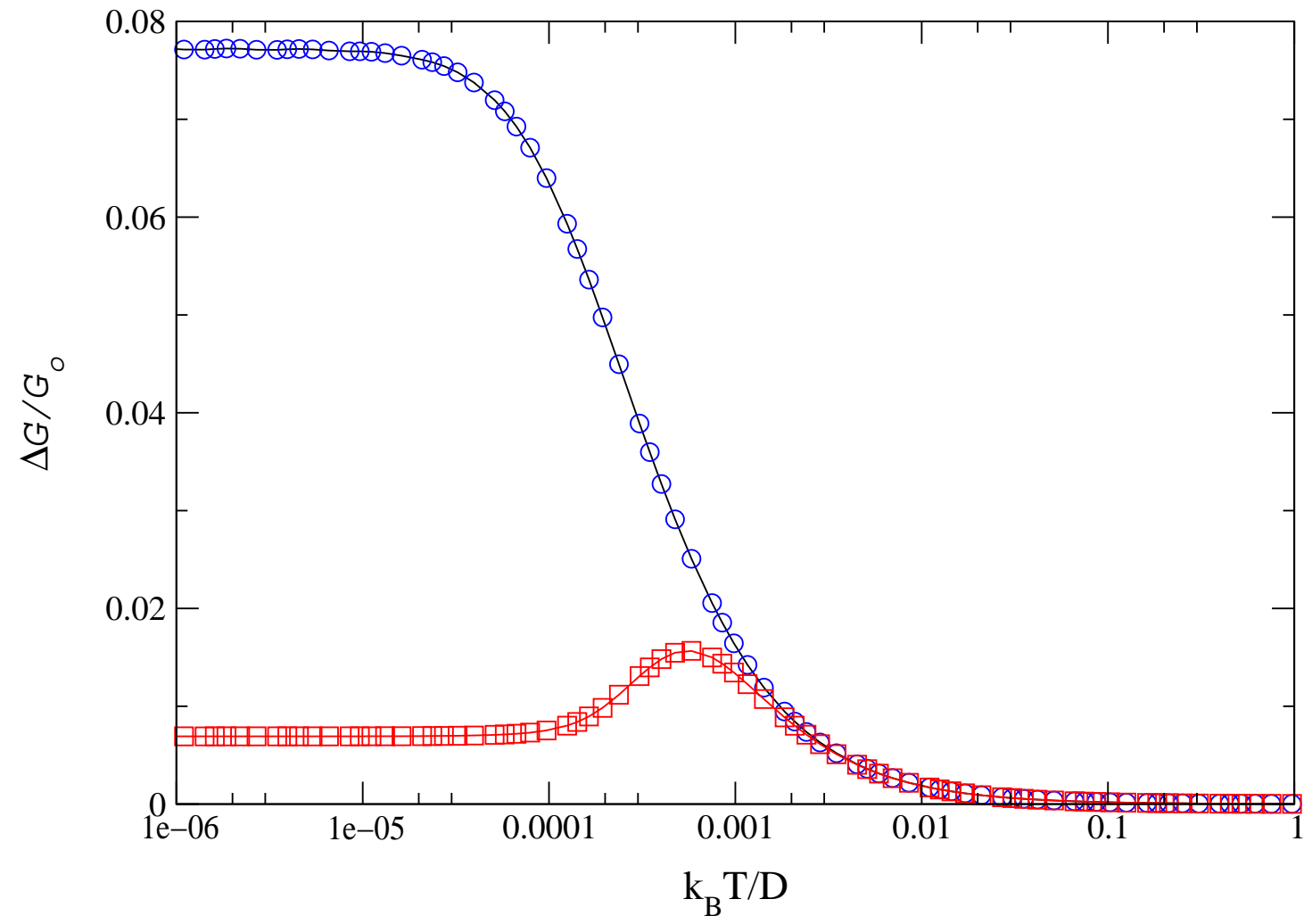

Figura 5.6: O gráfico compara dois casos para dois valores de $\varepsilon_{d}$, respectivamente $\varepsilon_{d}=0$ (curva azul) e $\varepsilon_{d}=-0,001 D$ (curva vermelha). A curva vermelha apresenta um pico nas proximidades do nível de energia $\left|\varepsilon_{d}\right|$, onde a densidade espectral de impureza é a pricipal responsável por este máximo. A curva azul não possui um máximo em temperaturas intermediárias, mas tem uma patamar intenso a baixas temperaturas. Os parâmetros usados foram $V=t=0,01 D, q_{F} R=\pi$, $N=25$ e $\Lambda=6$. 
impureza que assume um valor constante proporcional a $\frac{1}{\pi}$, o quê eleva o valor da condutância. A ausência de um pico em temperaturas intermediárias ocorre porque a densidade espectral de impureza e a derivada da distribuição de Fermi são funções centradas no nível de Fermi.

Os estudos basicamente desenvolvidos nas Figuras 5.3, 5.5 e 5.6 são análises termodinâmicas, onde a forma da condutância é determinada essencialmente por funções da temperatura. A influência da função $S(\varepsilon, R)$ ficará evidenciada numa análise em que a temperatura é mantida constante com a posição da ponta de prova variável.

Apresentamos também um estudo da condutância para uma temperatura fixa (próxima de zero) em função da distância entre a impureza atômica e a ponta de prova metálica do microscópio. As Figuras 5.7 e 5.8 mostram uma condutância oscilatória com a distância.

O espalhamento de elétrons de uma banda de condução por uma impureza atômica adsorvida gera um rearranjo de cargas refletido na densidade de carga do meio metálico. A variação da densidade de carga devido ao espalhamento de elétrons é uma quantidade oscilatória na distância. Essas oscilações na densidade de carga são chamadas oscilações de Friedel. A distribuição oscilatória de cargas no meio metálico induz uma corrente de tunelamento também oscilatória. 


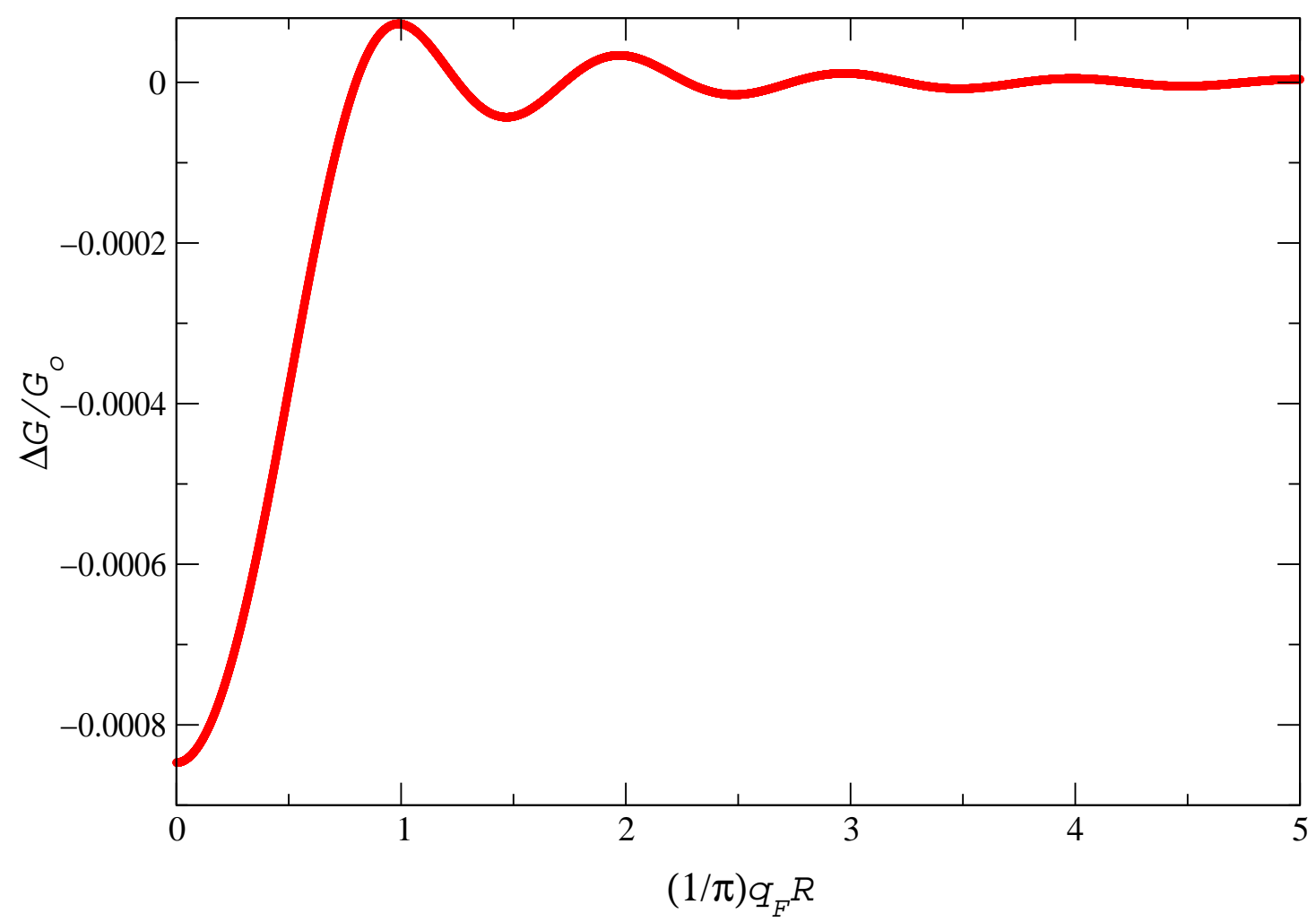

Figura 5.7: Gráfico da condutância contra distância com $k_{B} T=10^{-6} D$ gerado numericamente. Os parâmetros usados foram $N=25, \Lambda=3, V=t=0,01 D$, $\varepsilon_{d}=-0,01 D$. A oscilação da corrente em função da distância é uma das consequências das oscilações de Friedel. 


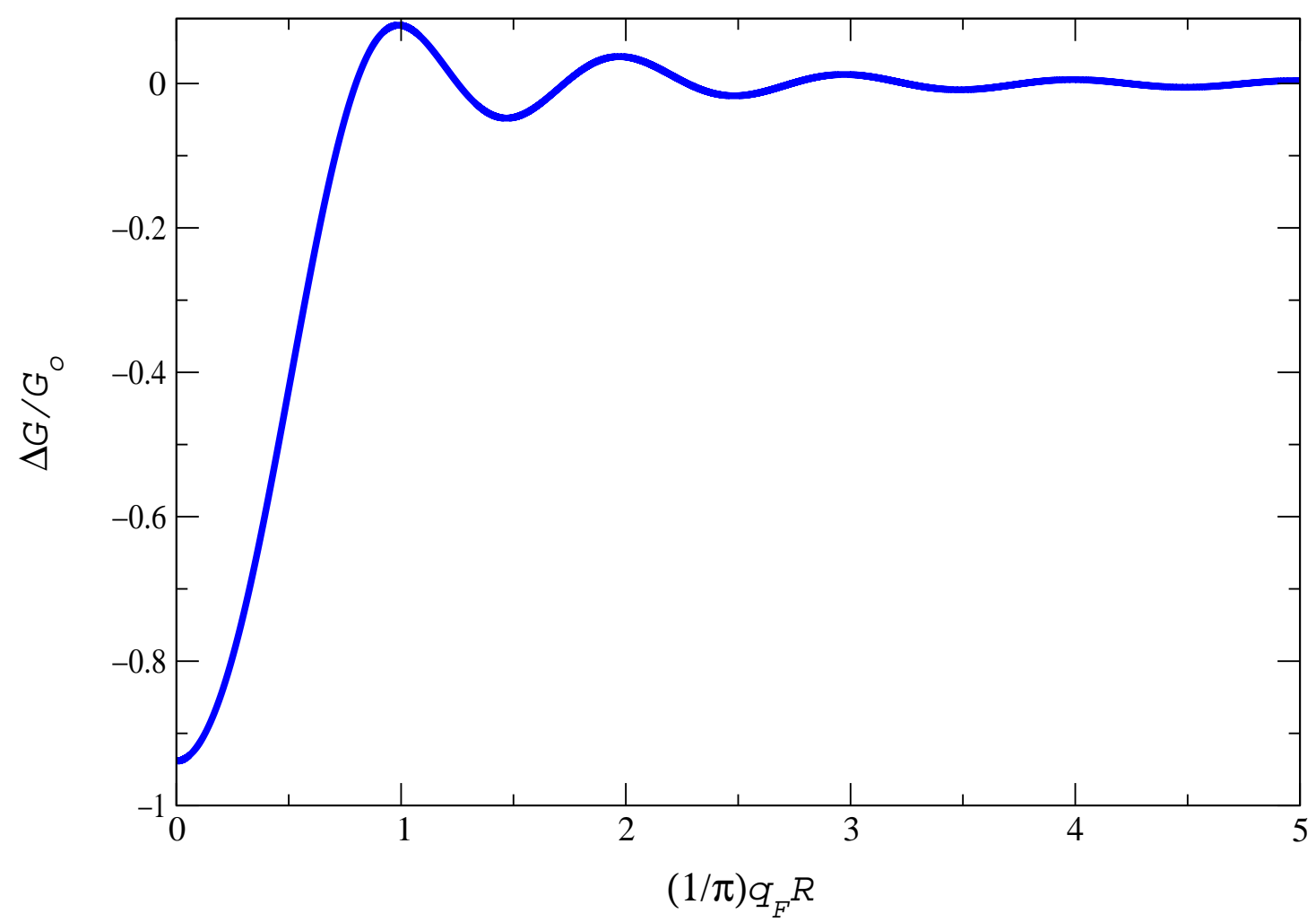

Figura 5.8: Gráfico da condutância contra distância com $k_{B} T=10^{-6} D$ gerado numericamente. Os parâmetros usados foram $N=25, \Lambda=3, V=t=0,01 D$ e $\varepsilon_{d}=0$. A oscilação da corrente em função da distância é uma das consequências das oscilações de Friedel. 


\section{Capítulo 6}

\section{Caso Interagente}

Vamos aqui desenvolver uma abordagem diferente das seções 5.1 e 5.2, onde confrontamos resultados exato e numérico (uso do GRN). O Hamiltoniano de Anderson completo, por considerar a correlação eletrônica $U$ no sítio da impureza, deixa de possuir uma forma quadrática e por isso não permite uma diagonalização exata como mostrada para o Modelo do Nível Ressonante. A única solução possível para isso é o uso de métodos numéricos.

Na ausência da correlação $U$ a densidade espectral de impureza possui uma ressonânica em $\varepsilon_{d}$. Já com a introdução do parâmetro $U$, há o aparecimento de máximos locais e o comportamento se afasta do conhecido para o modelo do Nível Ressonante. É nessa situação que surge o que se conhece por Ressonância de Kondo, uma aguda e estreita ressonância na densidade espectral exatamente no nível de Fermi. A Figura 6.1 ilustra bem esse pico. Reapresentamos os gráficos da densidade espectral de impureza nas proximidades da temperatura zero, com três valores distintos de $U$ gerados pelo Grupo de Renormalização Numérico.

\subsection{Diagonalização Iterativa}

A diagonalização do Hamiltoniano de Anderson com correlação eletrônica $(U \neq 0)$ não segue os mesmos passos que o caso do Nível Ressonante, onde 


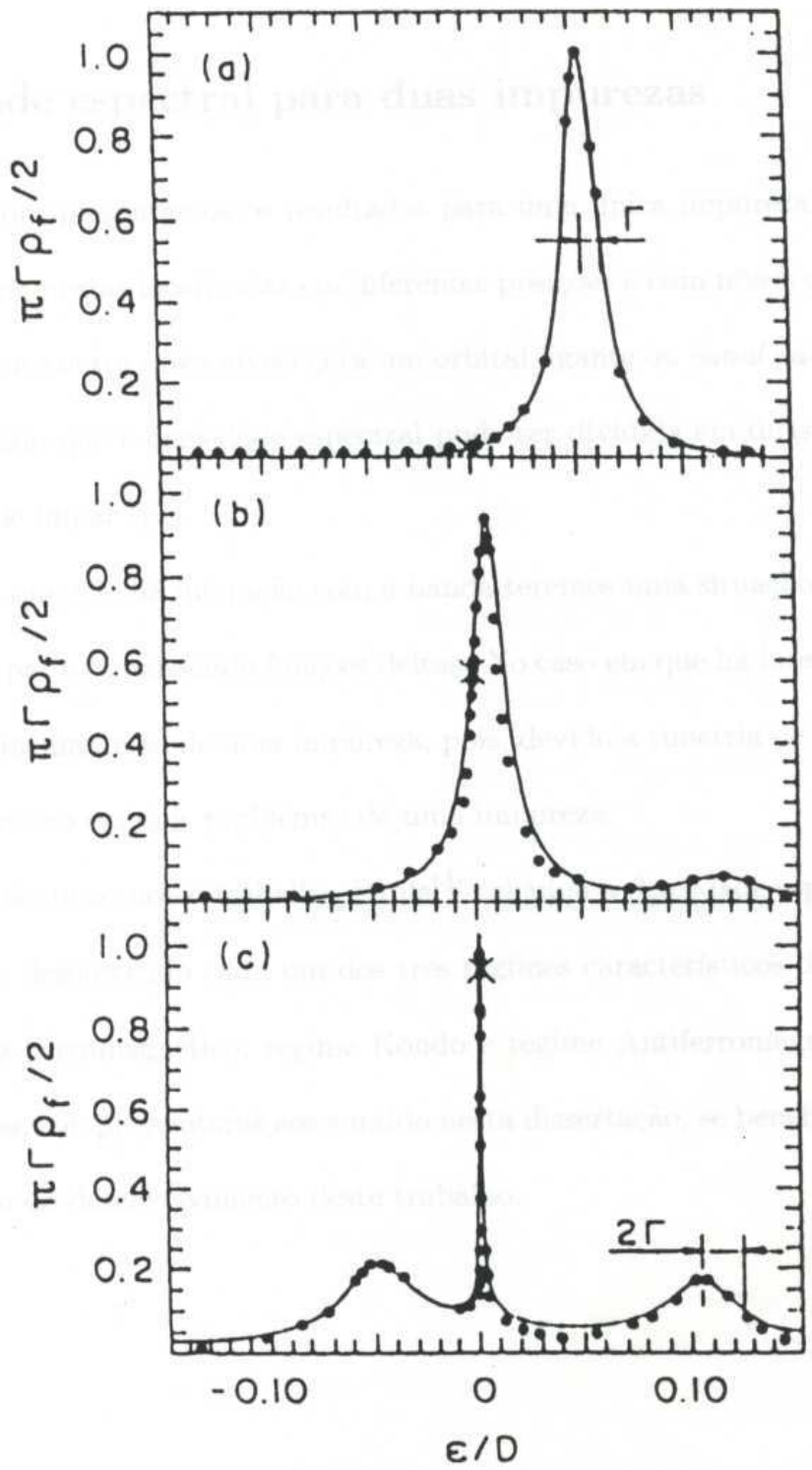

Figura 6.1: Densidade espectral $\rho_{f}$ para o modelo de Anderson com correlação $U$. As curvas foram geradas via Grupo de Renormalização Numérico assumindo $\varepsilon_{d}=-0.01 D$ e $\Gamma=0,01 D$. Foram usados os parâmetros $U=0,05 D, U=0,105 D$ e $U=0,15 D$ respectivamente. A linha sólida é uma combinação de Lorentzianas para ajustar os pontos de densidade criados numericamente. Quando $U \rightarrow 0$, a densidade espectral de impureza se aproxima de uma Lorentziana com meia largura igual à $\Gamma$ e centrada em $\varepsilon_{d}$. 
trabalhou-se com estados de uma partícula. O processo de diagonalização apóia-se completamente na relação de recorrência do Grupo de Renormalização (expressão (4.8)), ou seja, conhecidos os estados de muitos corpos e os autovalores de uma dada iteração, a transformação do Grupo permite determinar os próximos estados e também as autoenergias associadas da iteração seguinte. Esse algoritmo define uma diagonalização iterativa.

Primeiramente, antes de detalhar os estados de muitos corpos, definiremos os operadores de carga $Q$ total, os operadores de spin total $S$ e $S_{z}$ de uma iteração $N$. Sejam esses operadores definidos pelas operações

$$
\begin{gathered}
Q_{N}=\sum_{n, \sigma}^{N}\left(f_{n, \sigma}^{+} f_{n, \sigma}-1\right)+\sum_{\sigma}\left(c_{d, \sigma}^{+} c_{d, \sigma}-1\right), \\
\overrightarrow{S_{N}}=\sum_{n}^{N}\left(\begin{array}{cc}
f_{n, \uparrow}^{+} & f_{n, \downarrow}^{+}
\end{array}\right) \vec{\sigma}\left(\begin{array}{c}
f_{n, \uparrow} \\
f_{n, \downarrow}
\end{array}\right)+\left(\begin{array}{ll}
c_{d, \uparrow}^{+} & c_{d, \downarrow}^{+}
\end{array}\right) \vec{\sigma}\left(\begin{array}{c}
c_{d, \uparrow} \\
c_{d, \downarrow}
\end{array}\right), \\
S_{N, z}=\sum_{n=0}^{N} \frac{1}{2}\left(f_{n, \uparrow}^{+} f_{n, \uparrow}-f_{n, \downarrow}^{+} f_{n, \downarrow}\right)+\frac{1}{2}\left(c_{d, \uparrow}^{+} c_{d, \uparrow}-c_{d, \downarrow}^{+} c_{d, \downarrow}\right) .
\end{gathered}
$$$$
\text { e }
$$

Os operadores $Q_{N}, S_{N}^{2}$ e $S_{N, z}$ comutam com $H_{N}$ e por isso seus estados podem ser convenientemente identificados pelos números quânticos $Q, S$ e $S_{z}$. Assim, identifica-se um estado pelo ket $\left|Q, S, S_{z}\right\rangle_{N}$. Da relação de recorrência, vemos que a partir de um autoestado $\left|Q, S, S_{z}\right\rangle_{N}$ de $H_{N}$, podemos construir quatro estados de base de $H_{N+1}$, ou seja,

$$
\begin{array}{r}
\left|Q, S, S_{z}\right\rangle_{N}, \\
f_{(N+1), \uparrow}^{+}\left|Q, S, S_{z}\right\rangle_{N}, \\
f_{(N+1), \downarrow}^{+}\left|Q, S, S_{z}\right\rangle_{N} \\
54
\end{array}
$$




$$
f_{(N+1), \uparrow}^{+} f_{(N+1), \downarrow}^{+}\left|Q, S, S_{z}\right\rangle_{N}
$$

O operador $H_{N}$ possui $2(N+2)$ operadores de férmions (ocupados ou desocupados) e por isso há $2^{2(N+2)}$ autovetores na iteração $N$. Cada autovetor desse gera 4 estados de base para a iteração $N+1$, de modo que existe um total de $4 \times 2^{2(N+2)}$ vetores de base para $H_{N+1}$, que também são autovetores de $H_{N}$.

Esses autovetores de $H_{N}$, também autovetores de $Q_{N}, S_{N}^{2}$ e $S_{N, z}$, são

$$
\begin{gathered}
\left|Q, S, S_{z}, r, 1\right\rangle_{N+1}=\left|Q, S, S_{z}, r\right\rangle_{N}, \\
\left|Q, S, S_{z}, r, 2\right\rangle_{N+1}=\left(\frac{S+S_{z}}{2 S}\right)^{1 / 2} f_{(N+1), \uparrow}^{+}\left|Q-1, S-1 / 2, S_{z}-1 / 2, r\right\rangle_{N}+ \\
+\left(\frac{S-S_{z}}{2 S}\right)^{1 / 2} f_{(N+1), \downarrow}^{+}\left|Q-1, S-1 / 2, S_{z}+1 / 2, r\right\rangle_{N}, \\
\left|Q, S, S_{z}, r, 3\right\rangle_{N+1}=-\left(\frac{S-S_{z}+1}{2 S+2}\right)^{1 / 2} f_{(N+1), \uparrow}^{+}\left|Q-1, S+1 / 2, S_{z}-1 / 2, r\right\rangle_{N} \\
+\left(\frac{S+S_{z}+1}{2 S+2}\right)^{1 / 2} f_{(N+1), \downarrow}^{+}\left|Q-1, S+1 / 2, S_{z}+1 / 2, r\right\rangle_{N} \\
\left|Q, S, S_{z}, r, 4\right\rangle_{N+1}=f_{(N+1), \uparrow}^{+} f_{(N+1), \downarrow}^{+}\left|Q-2, S, S_{z}, r\right\rangle_{N} \cdot
\end{gathered}
$$


O índice $r$ é usado para distinguir um vetor de base de outro do mesmo subespaço $Q, S, S_{z}$. Os rótulos 1,2,3 e 4 indicam a forma como o vetor de base foi gerado.

Os estados (6.1) são autoestados de $H_{N}$,

$$
\begin{gathered}
N_{N+1}\left\langle 1, r, S_{z}, S, Q\left|H_{N}\right| Q, S, S_{z}, r, 1\right\rangle_{N+1}=E_{N}(Q, S, r), \\
{ }_{N+1}\left\langle 2, r, S_{z}, S, Q\left|H_{N}\right| Q, S, S_{z}, r, 2\right\rangle_{N+1}=E_{N}(Q-1, S-1 / 2, r), \\
{ }_{N+1}\left\langle 3, r, S_{z}, S, Q\left|H_{N}\right| Q, S, S_{z}, r, 3\right\rangle_{N+1}=E_{N}(Q-1, S+1 / 2, r), \\
N+1\left\langle 4, r, S_{z}, S, Q\left|H_{N}\right| Q, S, S_{z}, r, 4\right\rangle_{N+1}=E_{N}(Q-2, S, r) .
\end{gathered}
$$

Os elementos são determinados com a utilização conveniente do Teorema de Wigner-Eckart. Esse teorema garante que

$$
\left\langle Q^{\prime}, S^{\prime}, S_{z}^{,}, r^{\prime}\left|f_{\mu}^{+}\right| Q, S, S_{z}, r\right\rangle=\left\langle Q^{\prime}, S^{\prime}, r^{\prime}\left\|f^{+}\right\| Q, S, r\right\rangle \times\left\langle S, S_{z}, \frac{1}{2}, \mu \mid S^{\prime} S_{z}^{\prime}\right\rangle \text {, onde }
$$

os elementos $\left\langle S, S_{z}, \frac{1}{2}, \mu \mid S, S_{z}^{\prime}\right\rangle$ são os coeficientes de Clebsh-Gordan e os elementos $\left\langle Q^{\prime}, S^{\prime}, r^{\prime}\left\|f^{+}\right\| Q, S, r\right\rangle$ são elementos reduzidos independentes de $S_{z}$.

Os únicos diferentes de zero, considerando $S_{z}=S$ e omitindo $S_{z}$.

$$
\begin{aligned}
& { }_{N+1}\left\langle 1, r_{1}, S, Q\left|H_{N I}\right| Q, S, r_{2}, 2\right\rangle_{N+1}={ }_{N}\left\langle r_{1}, S, Q\left\|f_{N}^{+}\right\| Q-1, S-1 / 2, r_{2}\right\rangle_{N}, \\
& { }_{N+1}\left\langle 1, r_{1}, S, Q\left|H_{N I}\right| Q, S, r_{3}, 3\right\rangle_{N+1}={ }_{N}\left\langle r_{1}, S, Q\left\|f_{N}^{+}\right\| Q-1, S+1 / 2, r_{3}\right\rangle_{N},
\end{aligned}
$$




$$
\begin{array}{r}
N_{+1}\left\langle 2, r_{2}, S, Q\left|H_{N I}\right| Q, S, r_{4}, 4\right\rangle_{N+1}=\left(\frac{2 S}{2 S+1}\right)^{1 / 2} \times \\
\times_{N}\left\langle r_{2}, S-1 / 2, Q-1\left\|f_{N}^{+}\right\| Q-2, S, r_{4}\right\rangle_{N},
\end{array}
$$

$$
\begin{aligned}
& N_{+1}\left\langle 3, r_{3}, S,\right.\left.Q\left|H_{N I}\right| Q, S, r_{4}, 4\right\rangle_{N+1}=-\left(\frac{2 S+2}{2 S+1}\right)^{1 / 2} \times \\
& \times_{N}\left\langle r_{3}, S+1 / 2, Q-1\left\|f_{N}^{+}\right\| Q-2, S, r_{4}\right\rangle_{N} .
\end{aligned}
$$

Percebemos que ao conhecer os autovalores $E_{N}(Q . S, r)$ e os elementos $\left\langle\left|H_{N I}\right|\right\rangle$, podemos construir a matriz $H_{N+1}$ para diagonalizá-la, determinando seus autovetores e suas autoenergias. Devemos lembrar que a matriz $H_{N+1}$ possui subespaços $(Q, S)$. Para um dado subespaço (Q,S) (bloco dentro da matriz $\left.H_{N+1}\right)$, podemos escrever seus autoestados como sendo

$$
|Q, S, \omega\rangle_{N+1}=\sum_{r, i} U_{Q S}(\omega ; r i)|Q, S, r, i\rangle_{N+1}
$$

e os autovalores correspondentes são os elementos $E_{N+1}(Q, S, \omega)$. O índice $\omega$ diferencia autoestados de um mesmo subespaço $(Q, S)$.

O passo seguinte, a iteração $N+2$, além de se utilizar dos resultados $|Q, S, \omega\rangle_{N+1}$ e $E_{N+1}(Q, S, \omega)$, também precisa dos elementos $\left\langle\left\|f_{N+1}^{+}\right\|\right\rangle$.

Combinando os resultados anteriores, obtemos

$$
\left\langle\omega, S, Q\left\|f_{N+1}^{+}\right\| Q^{\prime}, S^{\prime}, \omega^{\prime}\right\rangle=\sum_{r} U_{Q S}(\omega ; r k) U_{Q, S},\left(\omega^{\prime} ; r 1\right)+
$$




$$
\pm\left(\frac{2 S^{\prime}+1}{2 s+1}\right)^{1 / 2} U_{Q S}(\omega ; r 4) U_{Q, S},\left(\omega^{\prime} ; r k^{\prime}\right)
$$

O sinal positivo é usado para $k=2$ e $k^{\prime}=3$ se $S=S^{\prime}+1 / 2$. O sinal negativo é usado para $k=3$ e $k^{\prime}=2$ se $S=S^{\prime}-1 / 2$.

E assim, procedemos sucessivamente.

\subsection{Fórmula da condutância para o caso intera- gente}

Retomemos o Hamiltoniano de Anderson completo, já escrito na base dos $f_{n}$ e pronto para ser diagonalizado pelo grupo de Renormalização Numérico,

$H_{N}=\Lambda^{(N-1) / 2}\left[\sum_{n=0}^{N-1} \xi_{n}^{z}\left(f_{n}^{+} f_{n+1}+\right.\right.$ h.c. $\left.)+\varepsilon_{d}^{z} c_{d}^{+} c_{d}+\sqrt{\widetilde{\Gamma}_{z}}\left(f_{o}^{+} c_{d}+h . c.\right)+U^{z} c_{d \uparrow}^{+} c_{d \uparrow} c_{d \downarrow}^{+} c_{d \downarrow}\right]$

onde $\xi_{n}^{z}=\frac{2}{1+\Lambda^{-1}} \frac{\varepsilon_{n}^{z}}{D} \Lambda^{z-1}, \varepsilon_{d}^{z}=\frac{2}{1+\Lambda^{-1}} \frac{\varepsilon_{d}}{D} \Lambda^{z-1}, \sqrt{\widetilde{\Gamma}_{z}}=\frac{2 \sqrt{2}}{1+\Lambda^{-1}} \frac{V}{D} \Lambda^{z-1}$ e $U^{z}=$ $\frac{2}{1+\Lambda^{-1}} \frac{U}{D} \Lambda^{z-1}$.

A implementação da seção 6.1 em nossa expressão de condutância precisa primeiramente de uma notação mais clara para os autoestados de muitos corpos do Hamiltoniano de Anderson, ou seja, é preciso explicitar os números quânticos que aparecem nos estados $|N\rangle$ e $|M\rangle$ do cálculo de $\left\langle N\left|c_{d}\right| M\right\rangle$. Nossa expressão de condutância, já identificando melhor os estados, fica igual a

$$
\frac{\Delta G(z)}{G_{o}}=\frac{\Gamma}{\pi k_{B} T} Z^{-1} \sum_{Q, J, n, m} \sum_{\sigma}(2 J+1) S(\varepsilon, R) \frac{\left|\left\langle Q+1, J, n\left\|c_{d, \sigma}^{+}\right\| Q, J-1 / 2, m\right\rangle\right|^{2}}{e^{\beta E_{n}(Q+1, J)}+e^{\beta E_{m}(Q, J-1 / 2)}}+
$$




$$
+\frac{\Gamma}{\pi k_{B} T} Z^{-1} \sum_{Q, J, n, m} \sum_{\sigma}(2 J+1) S(\epsilon, R) \frac{\left|\left\langle Q+1, J, n\left\|c_{d, \sigma}^{+}\right\| Q, J+1 / 2, m\right\rangle\right|^{2}}{e^{\beta E_{n}(Q+1, J)}+e^{\beta E_{m}(Q, J+1 / 2)}}
$$

onde a função Z é

$$
Z=\frac{\operatorname{senh}\left(\frac{1}{\Theta}\right)}{1+\cosh \left(\frac{1}{\Theta}\right)} \sum_{Q, J, n}(2 J+1) e^{-\beta E_{n}(Q, J)}
$$

$\operatorname{com} \varepsilon=E_{n}(Q+1, J)-E_{m}(Q, J-1 / 2)$ e $\epsilon=E_{n}(Q+1, J)-\beta E_{m}(Q, J+1 / 2)$, como sendo respectivamente energias de uma partícula. Os parâmetros J.Q e $n$ (ou $m$ ) são spin total, carga total e índice de estado respectivamente.

Por outro lado, a contribuição da parte real é aquela em que não há a conservação de energia dada pela Delta de Dirac, ou seja,

$$
\Re\left\{G_{d d}^{R}(\varepsilon)\right\}=\wp \sum_{N, M} Z^{-1}\left|\left\langle N\left|c_{d}\right| M\right\rangle\right|^{2} \frac{\left(e^{-\beta E_{N}}+e^{-\beta E_{M}}\right)}{\varepsilon+E_{N}-E_{M}} .
$$

A contribuição da expressão (6.4) já sabemos que é pequena e por isso mais uma vez iremos negligenciá-la. Vemos que os estados de muitos corpos do caso interagente trariam uma complicação adicional no cálculo numérico da condutância, pois todos os estados que diferem por mais de uma partícula seriam incorporados nesse cálculo.

\subsection{Resultados}

Nesta seção, apresentamos um estudo do sistema de STM a partir de um conjunto de valores para os parâmetros do Modelo. Na seção 5.3 não existia o termo de correlação $U$ e por isso, a análise baseava-se somente na variação da razão $\frac{\varepsilon_{d}}{V}$. Neste momento, com o aparecimento de mais um dado a considerar (a correlação $U$ ), é preciso fixar alguns parâmetros já empregados na seção do Modelo do Nível Ressonante (seção 5.3) e introduzir a energia $U$, verificando as modificações devido à sua presença.

O critério de comparação consiste em usar o valor de posição da ponta de pro- 
va como o que foi estabelecido nas análises do Modelo do Nível Ressonante, isto é, $q_{F} R=\pi$ e conservar o nível de energia de impureza também constante, como $\varepsilon_{d}=-0,01 D$. Assim, saberemos quanto um sistema com correlação eletrônica se afasta daquele que não a possui. A única diferença, exclusivamente por conveniência, reside na troca inicial do parâmetro $V=0,01 D$ por $V=0,02 D$. É claro que isso modifica a magnitude das condutâncias na comparação entre os casos sem e com correlação, porém isso não prejudica a interpretação dos resultados, pois a Física do Modelo de Anderson e a do processo de tunelamento dada pela fórmula de Kubo, estão refletidas nas formas das linhas de condutância.

Na Figura 6.2, mantivemos constantes a energia de impureza, a distância entre a ponta de prova metálica e a impureza, e resolvemos escolher a correlação $U=$ $-2 \varepsilon_{d}$ (Modelo de Anderson Simétrico). A Figura 6.2 apresenta duas curvas com seus respectivos valores de $V$ indicados e os comportamentos distintos associados.

Para se estabelecer uma comparação com o Modelo do Nível Ressonante devemos procurar inicialmente as características comuns dos dois casos, ou seja, se existem intervalos de temperatura onde as formas das condutâncias sejam semelhantes. Vemos que no caso da curva vermelha existe uma semelhança com o resultado da Figura 5.5 (Modelo do Nível Ressonante) até aproximandamente $\frac{k_{B} T}{D} \sim 10^{-3}$, isto é, existem valores de $V$ e $U$ que fazem do sistema com correlação passar a se comportar com se fosse um sem a correlação $U$. Na curva vermelha da Figura 6.2 está evidenciado que existe uma temperatura bem pequena na qual a condutância cresce rapidamente a um valor consideravelmente alto e se estabiliza num patamar. Assim, vemos notoriamente que a baixas temperaturas ocorre uma valor altíssimo de condutância. De forma muito semelhante, a curva azul também revelou um patamar elevado a temperaturas próximas de zero, com a diferença de não apresentar em temperaturas intermediárias uma máximo local tão salientado como aquele que existe na curva vermelha. A Física que explica o comportamento das curvas da Figura 6.2 pode ser entendida como a mesma que explica a condutância da Figura 5.5 até nas vizinhanças de $\frac{k_{B} T}{D} \sim 10^{-3}$. As curvas se 


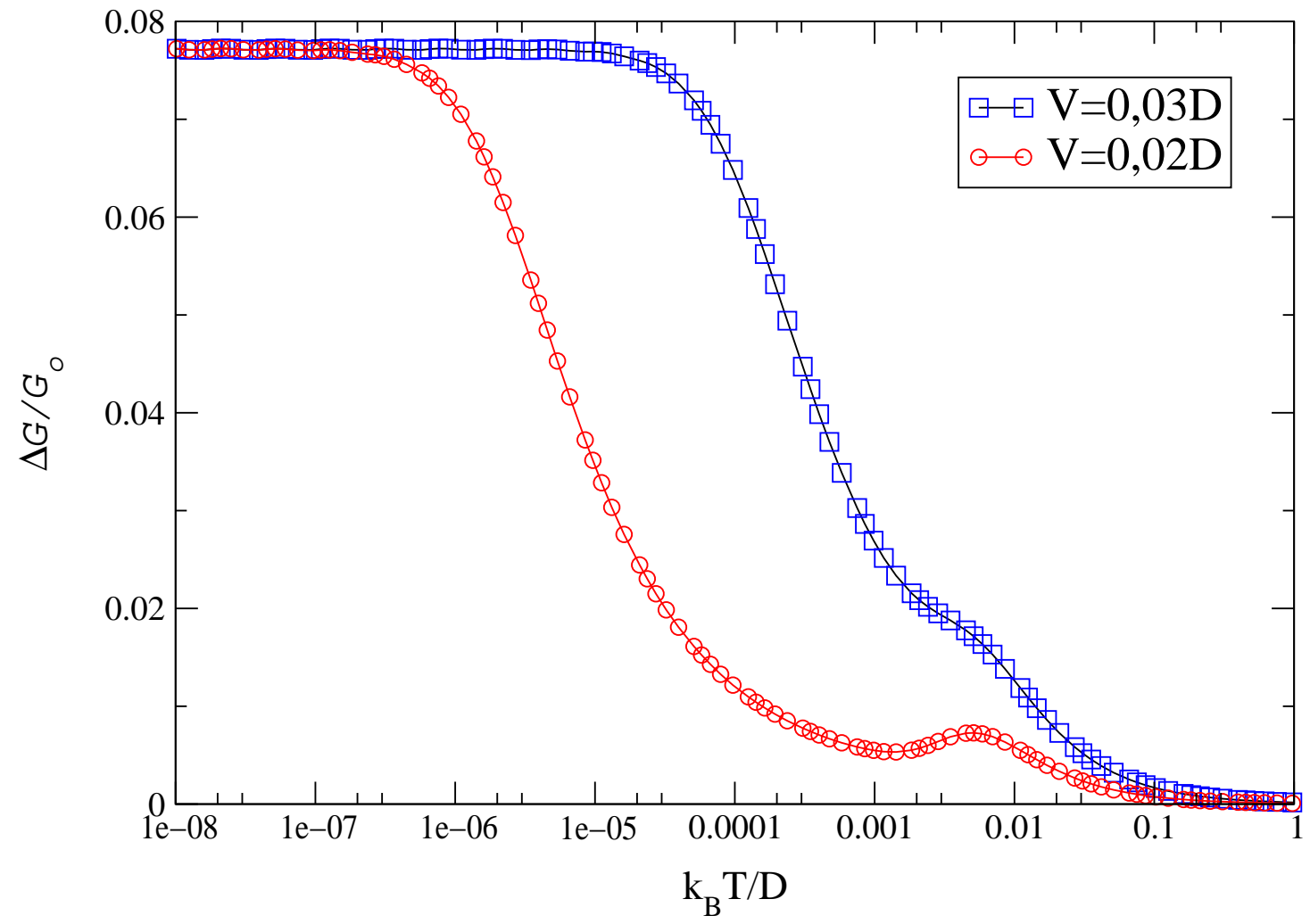

Figura 6.2: A curva vermelha apresenta um condutância que decai com o aumento de temperatura e que aumenta drasticamente com a diminuição da temperatura até um valor constante. Apresenta também um máximo local num intervalo de tempertaturas intermediárias. A curva azul possui comportamento semelhante. Em ambos os casos, a alta condutância se deve à ressonância de Kondo. Os parâmetros utilizados foram $\varepsilon_{d}=-0,01 D, U=0,02 D, q_{F} R=\pi, \Lambda=6 \mathrm{e}$ $N=46$. 
parecem com as do Modelo Ressonante porque a estas temperaturas o efeito Kondo é desprezível e não há ressonância de Kondo. Temperaturas inferiores a esse valor, revelam um comportamento não existente no Modelo do Nível Ressonante, isso significa que a forma da condutância nesse intervalo de temperaturas próximas de zero carrega um nova Física que sistemas sem correlação desconhecem. A razão da elevação da condutância das curvas da Figura 6.2 está diretamente associada à presença do efeito Kondo que é observado mais intensamente a baixas temperaturas. Assim como no Modelo do Nível Ressonante, a condutância depende diretamente da densidade espectral de impureza e da derivada da função de Fermi. Já sabemos que a segunda função seleciona os elétrons térmicos para o processo do tunelamento. Nesse caso, a densidade espectral de impureza não é só função de $\Gamma$ e $\varepsilon_{d}$, mas também da correlação $U$. Isso tudo muda a forma da densidade espectral para algo diferente de uma Lorentziana, a medida que $U$ fica bem maior que zero. Quando isso acontece, a densidade espectral de impureza possui uma ressonância intensa e estreita centrada no nível de Fermi (Figura 6.1), conhecida como ressonância de Kondo. Como já se sabe, a baixas temperaturas, a derivada de função de Fermi avalia toda a expressão da condutância na energia de Fermi e portanto, a expressão da condutância que é proporcional a densidade espectral, provoca uma condutância elevada, devido ao valor alto da densidade nesse regime de temperatura. Vemos que a elevada concentração de estados nas vizinhanças da energia de Fermi (ressonância Kondo) é a responsável direta pelo aumento da condutância. Concluímos também que o aumento do parâmetro $\mathrm{V}$ reduz o máximo local em temperaturas intermediárias, de modo que a tendência da condutância, com os parâmetros $\varepsilon_{d}, q_{F} R$ e $U$ usados na Figura 6.2 e mantidos constantes, é a de se aproximar de uma condutância típica do Modelo do Nível Ressonante $\operatorname{com} \varepsilon_{d}=0$, como aparece na Figura 5.6.

Analisemos um caso distinto da Figura 6.2. A Figura 6.3 mostra os resultados com correlação eletrônica $U=-\varepsilon_{d}$ e com os demais parâmetros utilizados na geração da Figura 6.2 . Neste ponto, desejamos investigar se a ressonância Kondo 


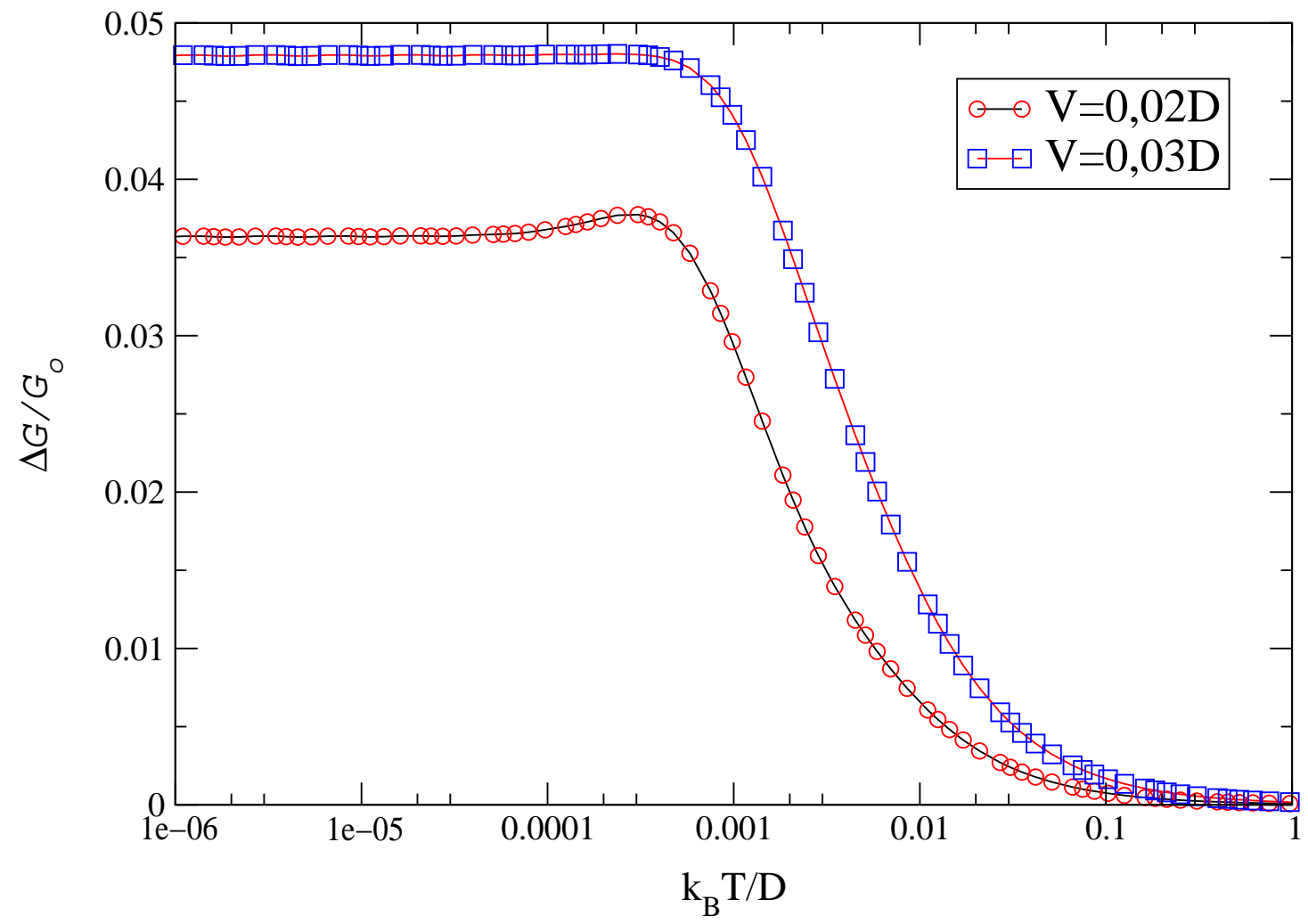

Figura 6.3: A curva vermelha mostra que a correlação $U=0,01 D$ com $\varepsilon_{d}=$ $-0,01 D$ faz com a condutância se comportar com se não existisse correlação e se o nível de energia de impureza tivesse um valor normalizado. A curva azul também manifesta uma forma similar do Modelo do Nível Ressonante. Os parâmetros do GRN usados foram $\Lambda=6$ e $N=46$.

influencia a condutância em baixas temperaturas como nos casos da Figura 6.2 para um caso diferente de correlação $U$.

A Figura 6.3, na verdade, ilustra duas configurações típicas do Modelo do Nível Ressonante. Vamos olhar primeiramente a curva vermelha da Figura 6.3, cujo comportamento já exibido na seção 5.3 possui uma Física bem clara, descrita pela competição entre a densidade espectral de impureza e a derivada da função de Fermi presentes na fórmula da condutância. Na curva vermelha notamos um máximo da condutância em temperaturas intermediárias, não nas proximidades do nível de energia do orbital de impureza como acontece no Modelo do Nível Ressonante, mas sim nas vizinhanças de uma energia diferente de $\varepsilon_{d}$. Essa dife- 
rença acontece por causa da presença da correlação $U$. Mesmo assim, a forma da linha da condutância é como no caso sem correlação. Assim, podemos inferir que a condutância de um sistema com correlação pode ser entendida como uma condutância de um sistema sem correlação com um nível de energia de impureza renormalizado. Isso também se aplica à curva azul da Figura 6.3, muito semelhante à curva azul da Figura 5.6.

O passo seguinte consiste em analisar a condutância em função da distância entre a ponta de prova do microscópio e a impureza para uma temperatura muito próxima de zero.

A Figura 6.4 apresenta três curvas com seus respectivos valores de correlação. Os parâmetros $\varepsilon_{d}$ e $V$ são mantidos constantes nessas três curvas.

Os resultados da Figura 6.4 mostram que as oscilações na corrente de tunelamento a baixas temperaturas diminuem com a diminuição da correlação $U$. Esse comportamento já era esperado, pois a densidade espectral de impureza tem sua forma muito semelhante à densidade do Modelo do Nível Ressonante, isto é, uma Lorentziana. É isso que faz com que as amplitudes de oscilação caiam com a diminuição da correlação.

Por outro lado, o aumento da correlação $U$ faz com que o número de estados fique muito grande em torno do nível de Fermi, isso significa que o sistema está no regime Kondo. Novamente, é a ressonânica Kondo da densidade espectral de impureza que faz com que as amplitudes de oscilação sejam maiores que no caso do Nível Ressonante. 


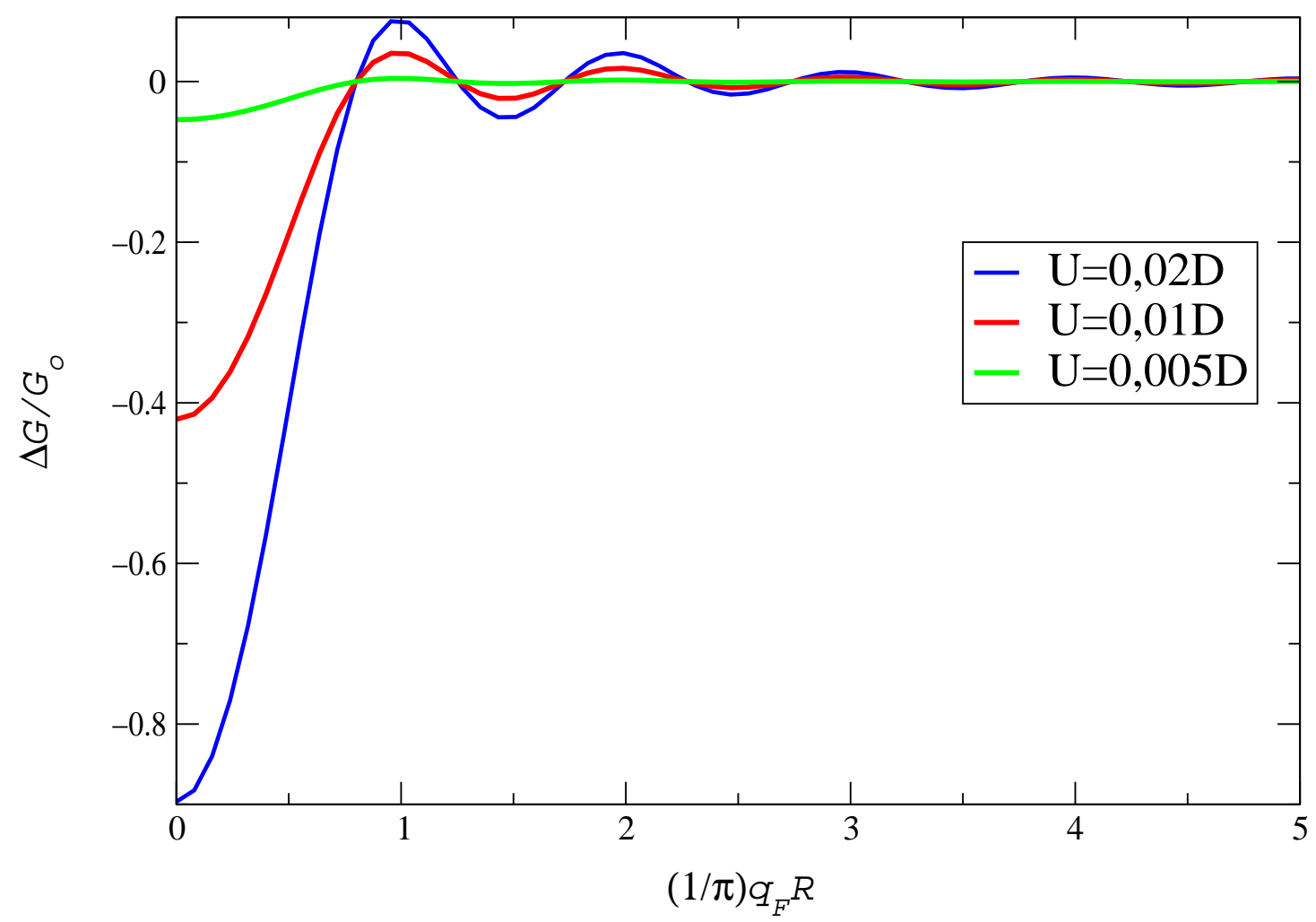

Figura 6.4: Condutância em função da distância numa temperatura próxima de zero para três casos interagentes. Vê-se que as amplitudes de oscilação diminuem com a diminuição do parâmetro de correlação $U$. Os parâmetros usados nas três curvas foram $\varepsilon_{d}=-0,01 D, V=0,02 D, \Lambda=6$ e $N=32$. 


\section{Capítulo 7}

\section{Conclusões}

Nesse trabalho mostramos que a expressão para a corrente de tunelamento (ou expressão (3.19) para a condutância) entre um meio metálico não magnético (metal hospedeiro) com uma impureza atômica adsorvida (magnética ou não magnética), descritos pelo Hamiltoniano de Anderson, e uma agulha metálica descrita pelo gaś de elétrons livres de Fermi, está consistente fisicamente. Essa consistência está evidenciada na expressão (3.19) através do termo constante (independente da distância entre a impureza e a ponta do microscópio), proporcional ao parâmetro $t^{2}$, e também através de outros dois termos proporcionais ao produto $V^{2} t^{2}$.

A dependência $t^{2}$ do primeiro termo faz com que ele se torne uma contribuição de fundo para a condutância, insensível à impureza no metal hospedeiro e caracterizando uma corrente de tunelamento de fundo devido somente ao acoplamento entre dois sistemas metálicos que trocam partículas.

O segundo termo é o que dá origem a influência da impureza no processo de tunelamento, já que a presença da amplitude $V^{2}$ está associada aos elétrons da banda de condução do metal hospedeiro espalhados pela impureza atômica conforme ilustrado na Figura 2.1. Verifica-se facilmente ao se fazer $V=0$ que a contribuição da impureza na corrente desaparece, restando apenas a corrente de fundo. Isso acontece quando a impureza deixa de ser um centro espalhador com 
o desacoplamento entre o nível de energia de impureza e a banda de condução.

A seção 5.3 inaugurou o estudo quantitativo da condutância de contato. Essa seção analisou um metal hospedeiro com uma impureza não magnética, isto é, a correlação eletrônica $U$ foi abandonada permitindo que o nível de energia de impureza ficasse duplamente ocupado. Estudos iniciais direcionaram-se para medir a influência da parte real da função de Green de impureza na condutância em função da temperatura. Foi mostrado através do resultado da Figura 5.3 a baixa contribuição dessa parte da função de Green, principalmente em temperaturas próximas de zero. Concluímos também que a implementação do Grupo de Renormalização Numérico apresentou-se safisfatória no estudo desse caso analiticamente solúvel, conforme ilustrado na Figura 5.5 .

Mencionamos a consistência física da expressão (3.19) argumentando os papéis desempenhados pelas amplitudes $t^{2}$ e $t^{2} V^{2}$. Uma maneira alternativa de compreender a pertubação dada pela impureza na medida da corrente, é observar as oscilações (oscilações de Friedel) primeiramente apresentadas na Figura 5.7 no estudo da condutância em função da distância, a uma temperatura próxima de zero. Vemos que as amplitudes de oscilação diminuem com o aumento da distância até se aproximarem de zero, isto é, em posições muito afastadas da impureza o tunelamento se dá como se a impureza não existisse, restando apenas a condutância de fundo. Na verdade nesse limite de grandes distâncias, esse era o comportamento esperado, pois os orbitais d são estados localizados e a superposição com as ondas planas dos estados dos elétrons da banda de condução deixam de ser relevantes.

A seção 6.3 por meio de estudos para diversos casos de correlação revelou na condutânica a baixas temperaturas um comportamento não presente no Modelo do Nível Ressonante. Esse regime de baixas temperaturas, conhecido como regime Kondo, faz com que propriedades termodinâmicas sejam regidas pela ressonância Kondo, identificada por um máximo na densidade espectral centrado na energia de Fermi, assim como mostra a Figura 6.1. O Efeito Kondo está bem nítido na 
Figura 6.2 para o caso do Modelo de Anderson Simétrico. A predominância da ressonância Kondo também ficou evidenciada na Figura 6.4, onde o aumento da correlação $U$ a baixas temperaturas, fez com que as amplitudes das oscilações de Friedel aumentassem. A abordagem final do caso interagente provou através da Figura 6.3 que pode haver uma seleção de parâmetros do modelo capaz de permitir que um sistema com correlação eletrônica $U$ se comporte como um sistema de Nível Ressonante com parâmetros renormalizados.

Concluímos a partir de análises termodinâmicas que a corrente de tunelamento total está intimamente ligada à correlação eletrônica no sítio de impureza. Verificamos também, que o deslocamento lateral da ponta de prova metálica do microscópio eletrônico mede uma corrente de tunelamento que é função do ponto onde se dá esse tunelamento. Isso permite entender a técnica de STM como uma forma indireta de detectar experimentalmente as oscilações de Friedel (dependentes da correlação), uma consequência do fenômeno de blindagem num meio metálico. Isso confirma que a perturbação da impureza magnética ou não magnética deve ser considerada durante a varredura dessa ponta por toda a superfície metálica, justamente por ela introduzir oscilações na corrente total de tunelamento.

Uma extensão desse trabalho seria acrescentar mais um canal de tunelamento no Hamiltoniano total, o tunelamento via orbital de impureza. Um termo que tornaria mais realísticas as medidas de condutância nas vizinhanças da impureza. 


\section{Apêndice A}

\section{Resultados importantes para a}

\section{fórmula de Kubo}

Aqui detalhamos algumas passagens matemáticas relevantes e utilizadas no Capítulo 3. O cálculo de $H_{t}(\tau)$ utilizou-se dos seguintes resultados.

Sabemos que

$e^{i \mu_{P} N_{P} \tau / \hbar} H_{t} e^{-i \mu_{P} N_{P} \tau / \hbar}=H_{t}+\left[N_{P}, H_{t}\right] \frac{i}{\hbar} \mu_{P} \tau+\frac{1}{2 !}\left[N_{P},\left[N_{P}, H_{t}\right]\right]\left(\frac{i}{\hbar} \mu_{P} \tau\right)^{2}+\cdots$

onde

$$
\begin{gathered}
{\left[N_{P}, H_{t}\right]=t \sum_{k \sigma q \delta} e^{i \vec{k} \cdot \overrightarrow{R_{2}}} c_{p q \delta}^{+} c_{s k \sigma}-h . c .} \\
{\left[N_{P},\left[N_{P}, H_{t}\right]\right]=H_{t} .}
\end{gathered}
$$

Logo, temos que 


$$
\begin{array}{r}
e^{i \mu_{P} N_{P} \tau / \hbar} H_{t} e^{-i \mu_{P} N_{P} \tau / \hbar}= \\
t \sum_{k \sigma q \delta} e^{-i \vec{k} \cdot \overrightarrow{R_{2}}} c_{s k \sigma}^{+} c_{p q \delta}\left(1-\frac{i}{\hbar} \mu_{P} \tau+\frac{1}{2 !}\left(\frac{i}{\hbar} \mu_{P} \tau\right)^{2}+\cdots\right)+ \\
+t \sum_{k \sigma q \delta} e^{i \vec{k} \cdot \overrightarrow{R_{2}}} c_{p q \delta}^{+} c_{s k \sigma}\left(1+\frac{i}{\hbar} \mu_{P} \tau+\frac{1}{2 !}\left(\frac{i}{\hbar} \mu_{P} \tau\right)^{2}+\cdots\right)
\end{array}
$$

$$
=t \sum_{k \sigma} \sum_{q \delta}\left(e^{-i \vec{k} \cdot \overrightarrow{R_{2}}} c_{s k \sigma}^{+} c_{p q \delta} e^{-\frac{i}{\hbar} \mu_{P} \tau}+\text { h.c. }\right) .
$$

A expansão do comutador que aparece na expressão (3.5) precisou dos passos seguintes. Começemos pela definição de média termodinâmica,

$$
\langle O\rangle=Z^{-1} \sum_{n} e^{-\beta E_{n}}\langle n|O| n\rangle .
$$

Fazendo a aplicação dessa definição,

$$
\begin{gathered}
\left\langle\left[c_{p k_{1}}^{+}(\tau) c_{s k_{2}}(\tau), c_{s k_{3}}^{+}\left(\tau^{\prime}\right) c_{p k_{4}}\left(\tau^{\prime}\right)\right]\right\rangle=Z^{-1} \sum_{n}\left\langle n\left|\left[c_{p k_{1}}^{+}(\tau) c_{s k_{2}}(\tau), c_{s k_{3}}^{+}\left(\tau^{\prime}\right) c_{p k_{4}}\left(\tau^{\prime}\right)\right]\right| n\right\rangle e^{-\beta E} \\
=Z^{-1} \sum_{n}\left\langle n\left|c_{p k_{1}}^{+}(\tau) c_{s k_{2}}(\tau) c_{s k_{3}}^{+}\left(\tau^{\prime}\right) c_{p k_{4}}\left(\tau^{\prime}\right)\right| n\right\rangle e^{-\beta E_{n}}+ \\
-Z^{-1} \sum_{n}\left\langle n\left|c_{s k_{3}}^{+}\left(\tau^{\prime}\right) c_{p k_{4}}\left(\tau^{\prime}\right) c_{p k_{1}}^{+}(\tau) c_{s k_{2}}(\tau)\right| n\right\rangle e^{-\beta E_{n}}
\end{gathered}
$$

Os sistemas Metal hospedeiro e ponta de prova metálica (descritos pelo Hamiltoniano de Anderson e pelo Hamiltoniano do gás de elétrons livres respectivamente) são independentes, ou seja, $Z=Z_{A} Z_{P}$, onde $Z_{A}$ e $Z_{P}$ são funções de partição do Essemble Grã-Canônico relativos ao Hamiltoniano de Anderson e da ponta de prova metálica respectivamente. Usamos também as identidades $\sum_{m}|m\rangle\langle m|=1$ e $|m\rangle=\left|f^{\prime}\right\rangle\left|g^{\prime}\right\rangle$, 


$$
\begin{aligned}
& Z_{A}^{-1} Z_{P}^{-1} \sum e^{-\beta\left(E_{f}^{A}+E_{g}^{P}\right)}\left\langle f\left|c_{s k_{2}}(\tau)\right| f^{\prime}\right\rangle\left\langle f^{\prime}\left|c_{s k_{3}}^{+}\left(\tau^{\prime}\right)\right| f\right\rangle\left\langle g\left|c_{p k_{1}}^{+}(\tau)\right| g^{\prime}\right\rangle\left\langle g^{\prime}\left|c_{p k_{4}}\left(\tau^{\prime}\right)\right| g\right\rangle+ \\
& -Z_{A}^{-1} Z_{P}^{-1} \sum e^{-\beta\left(E_{f}^{A}+E_{g}^{P}\right)}\left\langle f\left|c_{s k_{3}}^{+}\left(\tau^{\prime}\right)\right| f^{\prime}\right\rangle\left\langle f^{\prime}\left|c_{s k_{2}}(\tau)\right| f\right\rangle\left\langle g\left|c_{p k_{4}}\left(\tau^{\prime}\right)\right| g^{\prime}\right\rangle\left\langle g^{\prime}\left|c_{p k_{1}}^{+}(\tau)\right| g\right\rangle(\mathrm{A} .6)
\end{aligned}
$$

Utilizando-se da dependência temporal dos operadores e de que os elementos de matriz $\left\langle g^{\prime}\left|c_{p k_{4}}\right| g\right\rangle \neq 0$ para $c_{p k_{4}}|g\rangle=\left|g^{\prime}\right\rangle$ e $\left\langle g\left|c_{p k_{1}}^{+} c_{p k_{4}}\right| g\right\rangle \neq 0$ para $k_{1}=k_{4}$ $\operatorname{com} E_{g}^{P}=E_{g}^{P}-\left(\varepsilon_{k_{4}}^{P}-\mu_{P}\right)$ no primeiro termo acima.

No segundo termo acima, as condições são $\left\langle g\left|c_{p k_{4}}\right| g^{\prime}\right\rangle \neq 0$ para $c_{p k_{4}}\left|g^{\prime}\right\rangle=|g\rangle$ e $\left\langle g^{\prime}\left|c_{p k_{1}}^{+} c_{p k_{4}}\right| g^{\prime}\right\rangle \neq 0$ para $k_{1}=k_{4} \operatorname{com} E_{g}^{P}=E_{g}^{P}+\left(\varepsilon_{k_{4}}^{P}-\mu_{P}\right)$.

Ao se trocar $f$ por $f$, e $g$ por $g$ ' no segundo termo da expressão da corrente e incluindo o fator $i \hbar \omega$ para garantir a convergência da integração, restaurando $e^{i \vec{q} \cdot \overrightarrow{R_{2}}}$, chega-se à

$$
\begin{aligned}
I=\frac{2 e t^{2}}{\hbar} \Im \sum_{f f, k} Z_{A}^{-1}\left|\sum_{q \sigma}\left\langle f\left|e^{i \vec{q} \cdot \overrightarrow{R_{2}}} c_{s q \sigma}\right| f^{\prime}\right\rangle\right| & \frac{\left(e^{-\beta\left(E_{f}^{A},-\varepsilon_{k}^{P}+\mu_{P}\right)}-e^{-\beta E_{f}^{A}}\right)}{\left(E_{f}^{A}-E_{f}^{A}+\varepsilon_{k}^{P}\right)+i \hbar \omega} \times \\
\times & {\left[Z_{P}^{-1} \sum_{g} e^{-\beta E_{g}^{P}}\left\langle g\left|c_{p k}^{+} c_{p k}\right| g\right\rangle\right] . }
\end{aligned}
$$

Tomando-se o limite $\omega \rightarrow 0^{+}$e identificando o termo entre colchetes como a função distribuição de Fermi, a corrente se torna

$$
\begin{aligned}
I=-\frac{2 \pi e t^{2}}{\hbar} \sum_{f f, k} Z_{A}^{-1} \mid & \sum_{q \sigma}\left\langle f\left|e^{i \vec{q} \cdot \overrightarrow{R_{2}}} c_{s q \sigma}\right| f^{\prime}\right\rangle \mid \times \\
& \left(e^{-\beta\left(E_{f}^{A},-\varepsilon_{k}^{P}+\mu_{P}\right)}-e^{-\beta E_{f}^{A}}\right) f\left(\varepsilon_{k}^{P}-\mu_{P}\right) \quad \delta\left(E_{f}^{A}-E_{f}^{A}+\varepsilon_{k}^{P}\right) .
\end{aligned}
$$


A conservação da energia da distribuição Delta de Dirac, dá

$$
\begin{gathered}
I=-\frac{2 \pi e t^{2}}{\hbar} \sum_{f f, k} Z_{A}^{-1} e^{-\beta E_{f}^{A}}\left|\sum_{q \sigma}\left\langle f\left|e^{i \vec{q} \cdot \overrightarrow{R_{2}}} c_{s q \sigma}\right| f^{\prime}\right\rangle\right|^{2}\left(e^{-\beta \mu_{P}}-1\right) f\left(\varepsilon_{k}^{P}-\mu_{P}\right) \times \\
\times \delta\left(E_{f}^{A}-E_{f}^{A}+\varepsilon_{k}^{P}\right) .
\end{gathered}
$$

A diferença de potencial elétrico $\phi$ é muito pequena, e por isso trabalharemos em regime linear, ou seja, $e^{-\beta \mu_{P}} \cong 1-\beta \mu_{P}=1-\beta e \phi$ e $f\left(\varepsilon_{k}^{P}-\mu_{P}\right) \cong f\left(\varepsilon_{k}^{P}\right)-$ $\phi \frac{\partial}{\partial \varepsilon_{k}^{P}} f\left(\varepsilon_{k}^{P}\right)$.

Utilizando-se de mais algumas identidades

$$
\begin{aligned}
& e^{-\beta E_{f}^{A}} \delta\left(E_{f}^{A}-E_{f}^{A}+\varepsilon_{k}^{p}\right)=\left(1-f\left(\varepsilon_{k}^{p}\right)\right)\left(e^{-\beta E_{f}^{A}}+e^{-\beta E_{f}^{A}}\right) \times \\
& \times \delta\left(E_{f}^{A}-E_{f}^{A}+\varepsilon_{k}^{p}\right) \\
& f\left(\varepsilon_{k}^{p}\right) \times\left(1-f\left(\varepsilon_{k}^{p}\right)\right)=-\frac{1}{\beta} \frac{\partial}{\partial \varepsilon_{k}^{p}} f\left(\varepsilon_{k}^{p}\right)
\end{aligned}
$$

Assim, chega-se a uma expressão de corrente até primeira ordem em $\phi$,

$$
\begin{aligned}
I=\frac{2 \pi e^{2} t^{2}}{\hbar} \phi \sum_{k f f} Z_{A}^{-1}\left|\sum_{q \sigma}\left\langle f\left|e^{i \vec{q} \cdot \overrightarrow{R_{2}}} c_{s q \sigma}\right| f^{\prime}\right\rangle\right|^{2}\left(e^{-\beta E_{f}^{A}}+e^{-\beta E_{f}^{A},}\right) \times & \\
& \times\left(-\frac{\partial}{\partial \varepsilon_{k}^{p}} f\left(\varepsilon_{k}^{p}\right)\right) \quad \delta\left(E_{f}^{A}-E_{f}^{A}+\varepsilon_{k}^{p}\right) .
\end{aligned}
$$




\section{Apêndice B}

\section{Funções de Green a temperatura $\mathrm{T}$}

\section{no cálculo da fórmula de Kubo}

A função de Green retardada temporal associada aos operadores $a, b$ é

$$
\begin{aligned}
& G_{a b}^{R}(t)=-\frac{i}{\hbar} \theta(t) \sum_{N} Z^{-1} e^{-\beta E_{N}}\left\langle N\left|\left[a(t), b^{+}(0)\right]_{+}\right| N\right\rangle= \\
= & -\frac{i}{\hbar} \theta(t) \sum_{N, M} Z^{-1}\langle N|a| M\rangle\left\langle M\left|b^{+}\right| N\right\rangle\left(e^{-\beta E_{N}}+e^{-\beta E_{M}}\right) e^{\frac{i}{\hbar}\left(E_{N}-E_{M}\right) t}
\end{aligned}
$$

onde foi usado $a(t)=e^{\frac{i H}{\hbar} t} a(0) e^{-\frac{i H}{\hbar} t}$ e $b(t)=e^{\frac{i H}{\hbar} t} b(0) e^{-\frac{i H}{\hbar} t}$.

A função de Green retardada no espaço de enegia é obtida pela transformada de Fourier

$$
G_{a b}^{R}(\varepsilon)=\int G_{a b}^{R}(t) e^{\frac{i}{\hbar}(\varepsilon+i \delta) t} d t=\sum_{N, M} Z^{-1}\langle N|a| M\rangle\left\langle M\left|b^{+}\right| N\right\rangle \frac{\left(e^{-\beta E_{N}}+e^{-\beta E_{M}}\right)}{\varepsilon+E_{N}-E_{M}+i \delta} .
$$

A transformada sobre $\frac{\partial}{\partial t} G_{a b}^{R}(t)$ é

$$
\int \frac{\partial}{\partial t} G_{a b}^{R}(t) e^{\frac{i}{\hbar}(\varepsilon+i \delta) t} d t=-\frac{i}{\hbar}(\varepsilon+i \delta) G_{a b}^{R}(\varepsilon) .
$$


Para o caso da função de Green avançada, temos

$$
\begin{array}{r}
G_{a b}^{A}(t)=\frac{i}{\hbar} \theta(-t) \sum_{N} Z^{-1} e^{-\beta E_{N}}\left\langle N\left|\left[a^{+}(0), b(t)\right]_{+}\right| N\right\rangle= \\
=\frac{i}{\hbar} \theta(-t) \sum_{N, M} Z^{-1}\left\langle M\left|a^{+}\right| N\right\rangle\langle N|b| M\rangle\left(e^{-\beta E_{N}}+e^{-\beta E_{M}}\right) e^{\frac{i}{\hbar}\left(E_{N}-E_{M}\right) t}
\end{array}
$$

A transformada de Fourier para este caso é

$G_{a b}^{A}(\varepsilon)=\int G_{a b}^{A}(t) e^{\frac{i}{\hbar}(\varepsilon-i \delta) t} d t=\sum_{N, M} Z^{-1}\left\langle M\left|a^{+}\right| N\right\rangle\langle N|b| M\rangle \frac{\left(e^{-\beta E_{N}}+e^{-\beta E_{M}}\right)}{\varepsilon+E_{N}-E_{M}-i \delta}$.

A transformada sobre $\frac{\partial}{\partial t} G_{a b}^{A}(t)$ é

$$
\int \frac{\partial}{\partial t} G_{a b}^{A}(t) e^{\frac{i}{\hbar}(\varepsilon-i \delta) t} d t=-\frac{i}{\hbar}(\varepsilon-i \delta) G_{a b}^{A}(\varepsilon)
$$

Concluímos portanto

$$
G_{a b}^{R}(\varepsilon)=\left(G_{a b}^{A}(\varepsilon)\right)^{+}
$$

A seguir, mostraremos alguns passos algébricos que permitirão escrever a condutância em uma forma mais adequada para cálculo.

Considere primeiramente a função de Green retardada temporal

$$
G^{R}(t)=-\frac{i}{\hbar} \theta(t) \sum_{N} Z^{-1} e^{-\beta E_{N}}\left\langle N\left|\left[O(t), O^{+}(0)\right]_{+}\right| N\right\rangle
$$

onde

$$
O=\sum_{k, \sigma} e^{i \vec{k} \cdot \vec{R}_{2}} c_{s k \sigma}
$$


Sua derivada é

$$
\begin{aligned}
& \frac{\partial}{\partial t} G^{R}(t)=-\frac{i}{\hbar} \delta(t) \sum_{N} Z^{-1} e^{-\beta E_{N}}\left\langle N\left|\left[O(t), O^{+}(0)\right]_{+}\right| N\right\rangle+ \\
& -\frac{i}{\hbar} \theta(t) \sum_{N} Z^{-1} e^{-\beta E_{N}}\left\langle N\left|\left[\frac{\partial}{\partial t} O(t), O^{+}(0)\right]_{+}\right| N\right\rangle= \\
& =\sum_{k, q} e^{i(\vec{k}-\vec{q}) \cdot \vec{R}_{2}}\left\{-\frac{i}{\hbar} \delta(t) \sum_{N} Z^{-1} e^{-\beta E_{N}}\left\langle N\left|\left[c_{s k}(t), c_{s q}^{+}(0)\right]_{+}\right| N\right\rangle\right\}+ \\
& +\sum_{k, q} e^{i(\vec{k}-\vec{q}) \cdot \vec{R}_{2}}\left\{-\frac{i}{\hbar} \theta(t) \sum_{N} Z^{-1} e^{-\beta E_{N}}\left\langle N\left|\left[\frac{\partial}{\partial t} c_{s k}(t), c_{s q}^{+}(0)\right]_{+}\right| N\right\rangle\right\}
\end{aligned}
$$

Nesse momento usamos a equação de movimento

$$
i \hbar \frac{\partial}{\partial t} c_{s k}(t)=\left[c_{s k}(t), H(t)\right]=\varepsilon_{k}^{s} c_{s k}(t)+V e^{-i \vec{k} \cdot \vec{R}_{1}} c_{d}(t)
$$

Assumimos a origem na impureza e obtemos

$$
\begin{gathered}
\frac{\partial}{\partial t} G^{R}(t)=\sum_{k, q} e^{i(\vec{k}-\vec{q}) \cdot \vec{R}_{2}}\left\{-\frac{i}{\hbar} \delta(t) \sum_{N} Z^{-1} e^{-\beta E_{N}}\left\langle N\left|\left[c_{s k}(t), c_{s q}^{+}(0)\right]_{+}\right| N\right\rangle\right\}+ \\
+\frac{1}{i \hbar} \sum_{k, q} \varepsilon_{k}^{s} e^{i(\vec{k}-\vec{q}) \cdot \vec{R}_{2}} G_{k q}^{R}(t)+\frac{1}{i \hbar} \sum_{k, q} V e^{i(\vec{k}-\vec{q}) \cdot \vec{R}_{2}} G_{d q}^{R}(t) \cdot
\end{gathered}
$$

No resultado (B.11), já identificamos as funções de Green retardadas

$$
G_{k q}^{R}(t)=-\frac{i}{\hbar} \theta(t) \sum_{N} Z^{-1} e^{-\beta E_{N}}\left\langle N\left|\left[c_{s k}(t), c_{s q}^{+}(0)\right]_{+}\right| N\right\rangle
$$

e

$$
G_{d q}^{R}(t)=-\frac{i}{\hbar} \theta(t) \sum_{N} Z^{-1} e^{-\beta E_{N}}\left\langle N\left|\left[c_{d}(t), c_{s q}^{+}(0)\right]_{+}\right| N\right\rangle .
$$


O procedimento para determinar o comportamento explícito de $G_{k k}^{R}(\varepsilon)$ e $G_{k q}^{R}(\varepsilon)$ é análogo.

Sabemos que

$$
G_{k q}^{R}(t)=-\frac{i}{\hbar} \theta(t) \sum_{N} Z^{-1} e^{-\beta E_{N}}\left\langle N\left|\left[c_{s k}(t), c_{s q}^{+}(0)\right]_{+}\right| N\right\rangle
$$

e

$$
\begin{aligned}
\frac{\partial}{\partial t} G_{k q}^{R}(t)= & -\frac{i}{\hbar} \delta(t) \sum_{N} Z^{-1} e^{-\beta E_{N}}\left\langle N\left|\left[c_{s k}(t), c_{s q}^{+}(0)\right]_{+}\right| N\right\rangle+ \\
& -\frac{i}{\hbar} \theta(t) \sum_{N} Z^{-1} e^{-\beta E_{N}}\left\langle N\left|\left[\frac{\partial}{\partial t} c_{s k}(t), c_{s q}^{+}(0)\right]_{+}\right| N\right\rangle,
\end{aligned}
$$

ou seja,

$$
\frac{\partial}{\partial t} G_{k q}^{R}(t)=-\frac{i}{\hbar} \delta(t) \sum_{N} Z^{-1} e^{-\beta E_{N}}\left\langle N\left|\left[c_{s k}(t), c_{s q}^{+}(0)\right]_{+}\right| N\right\rangle+\frac{i}{\hbar} \varepsilon_{k}^{s} G_{k q}^{R}(t)+\frac{i}{\hbar} V G_{d q}^{R}(t) .
$$

Lembrando que $G_{d q}^{R}(\varepsilon)=\left(G_{d q}^{A}(\varepsilon)\right)^{+}$, basta então determinar $G_{d q}^{A}(\varepsilon)$.

A função de Green que precisamos é

$$
G_{d q}^{A}(t)=\frac{i}{\hbar} \theta(-t) \sum_{N} Z^{-1} e^{-\beta E_{N}}\left\langle N\left|\left[c_{d}^{+}(0), c_{s q}(t)\right]_{+}\right| N\right\rangle .
$$

Fazendo a derivada parcial com relação ao tempo, obtemos

$$
\begin{aligned}
\frac{\partial}{\partial t} G_{d q}^{A}(t) & =-\frac{i}{\hbar} \delta(t) \sum_{N} Z^{-1} e^{-\beta E_{N}}\left\langle N\left|\left[c_{d}^{+}(0), c_{s q}(t)\right]_{+}\right| N\right\rangle+ \\
& +\frac{i}{\hbar} \theta(-t) \sum_{N} Z^{-1} e^{-\beta E_{N}}\left\langle N\left|\left[c_{d}^{+}(0), \frac{\partial}{\partial t} c_{s q}(t)\right]_{+}\right| N\right\rangle .
\end{aligned}
$$

No espaço de energia essa função fica finalmente igual à 


$$
G_{d q}^{A}(\varepsilon)=\frac{V}{\varepsilon-\varepsilon_{q}^{s}-i \delta} G_{d d}^{A}(\varepsilon) .
$$




\section{Apêndice C}

\section{Cálculo de integrais}

As energias cinéticas são

$$
\varepsilon_{q}^{S}=\frac{\hbar^{2} q^{2}}{2 m}-\mu_{s}
$$

e

$$
\varepsilon=\frac{\hbar^{2} k^{2}}{2 m}-\mu_{P}
$$

Vamos resolver primeiramente,

$\wp \sum_{\vec{q}} \frac{e^{i \vec{q} \cdot \vec{R}}}{\varepsilon-\varepsilon_{q}^{S}}=\frac{\Omega_{o}}{(2 \pi)^{3}} \wp \int \frac{e^{i \vec{q} \cdot \vec{R}}}{\varepsilon-\varepsilon_{q}^{S}} d^{3} q=\frac{\Omega_{o}}{(2 \pi)^{2}} \wp \int \frac{1}{\varepsilon-\varepsilon_{q}^{S}}\left(\int e^{i q \operatorname{Rcos} \theta} \operatorname{sen} \theta d \theta\right) q^{2} d q$

$$
=-\rho_{S}\left(\varepsilon_{F}\right) \frac{1}{q_{F} R} \wp \int \frac{\operatorname{sen}(q R)}{\varepsilon_{q}^{S}-\varepsilon} d \varepsilon_{q}^{S} .
$$

Usamos na expressão (C.3) $\rho_{S}\left(\varepsilon_{q}^{S}\right)=\frac{\Omega_{o}}{2 \pi^{2}} q^{2} \frac{d q}{d \varepsilon_{q}^{S}}$.

Fazendo-se as substiuições $\epsilon=\frac{\varepsilon_{q}^{S}}{D}, D \approx \mu_{S}=\frac{\hbar^{2} q_{F}^{2}}{2 m}, \rho_{S}\left(\varepsilon_{F}\right) \approx \frac{1}{D}$ e linearizando a relação de dispersão em torno de $\mu_{S}$, chegamos a 


$$
\sum_{\vec{q}} \frac{e^{i \vec{q} \cdot \vec{R}}}{\varepsilon-\varepsilon_{q}^{S}}=-\frac{1}{D} \frac{1}{q_{F} R} \int_{-D}^{+D} \frac{\operatorname{sen}\left[q_{F} R\left(1+\frac{1}{2} \frac{\varepsilon_{q}^{S}}{D}\right)\right]}{\varepsilon_{q}^{S}-\varepsilon} d \varepsilon_{q}^{S}
$$

Trocando $\frac{\varepsilon}{D}$ por $\varepsilon$ e $\frac{\varepsilon_{q}^{S}}{D}$ por $\epsilon$, obtemos

$$
\sum_{\vec{q}} \frac{e^{i \vec{q} \cdot \vec{R}}}{\varepsilon-\varepsilon_{q}^{S}}=-\frac{1}{D} \frac{\Phi(\varepsilon)}{q_{F} R}
$$

onde

$$
\Phi(\varepsilon)=\wp \int_{-1}^{+1} \frac{\operatorname{sen}\left[q_{F} R\left(1+\frac{1}{2} \epsilon\right)\right]}{\epsilon-\varepsilon} d \epsilon
$$

Na resolução de $\Phi(\varepsilon)$, vamos fazer uma mudança de variável do tipo $x=\epsilon-\varepsilon$ e representar o valor principal por

$$
\begin{gathered}
\Phi(\varepsilon)=\wp \int_{-(1+\varepsilon)}^{+(1-\varepsilon)} \frac{\operatorname{sen}\left[q_{F} R\left(1+\frac{1}{2} \varepsilon+\frac{1}{2} x\right)\right]}{x} d x \\
\equiv \lim _{\delta \rightarrow 0}\left[\int_{-(1+\varepsilon)}^{-\delta} \frac{\operatorname{sen}\left[q_{F} R\left(1+\frac{1}{2} \varepsilon+\frac{1}{2} x\right)\right]}{x} d x+\int_{\delta}^{+(1-\varepsilon)} \frac{\operatorname{sen}\left[q_{F} R\left(1+\frac{1}{2} \varepsilon+\frac{1}{2} x\right)\right]}{x} d x\right]
\end{gathered}
$$

Expandindo a função seno numa série de Taylor em torno de $x=0$, temos

$$
\begin{array}{r}
\Phi(\varepsilon)=\left[\ln \frac{1-\varepsilon}{1+\varepsilon}\right] \operatorname{sen}\left[q_{F} R\left(1+\frac{1}{2} \varepsilon\right)\right]+ \\
\cos \left[q_{F} R\left(1+\frac{1}{2} \varepsilon\right)\right] \sum_{n, \text { impar }} \alpha_{n} \frac{1}{n !}\left(\frac{q_{F} R}{2}\right)^{n} \frac{1}{n}\left[(1-\varepsilon)^{n}+(1+\varepsilon)^{n}\right]+ \\
+\operatorname{sen}\left[q_{F} R\left(1+\frac{1}{2} \varepsilon\right)\right] \sum_{n, \text { par }} \beta_{n} \frac{1}{n !}\left(\frac{q_{F} R}{2}\right)^{n} \frac{1}{n}\left[(1-\varepsilon)^{n}-(1+\varepsilon)^{n}\right],
\end{array}
$$


O parâmetro $\alpha_{n}$ é +1 para $\mathrm{n}=1,5,9, \ldots$ e -1 para $\mathrm{n}=3,7,11, \ldots$, enquanto que $\beta_{n}$ é -1 para $n=2,6,10$ e +1 para $n=4,8,12, \ldots$.

De forma análoga

$$
\sum_{\vec{q}} e^{i \vec{q} \cdot \vec{R}} \delta\left(\varepsilon-\varepsilon_{q}^{s}\right)=\frac{1}{D} \frac{\operatorname{sen}\left[q_{F} R\left(1+\frac{1}{2} \varepsilon\right)\right]}{q_{F} R} .
$$

Outra integral também importante é

$$
\int_{-D}^{+D}\left(-\frac{\partial}{\partial \varepsilon} f\right) d \varepsilon=-f+\left.C\right|_{-D} ^{+D}=-\frac{1}{1+e^{\beta \varepsilon}}+\left.C\right|_{-D} ^{+D}=\frac{\operatorname{senh}(\beta D)}{1+\cosh (\beta D)}
$$




\section{Referências Bibliográficas}

[1] Li. J., Schneider W-D. Kondo Scattering Observed at a Single Magnetic Impurity. Phys. Rev. Lett. v.80,n.13,p.2893, 1998.

[2] A.C. Hewson. The Kondo Problem to Heavy Fermions, Cambridge University Press, 1993.

[3] Mahan G.D. Many Particle-Physics, Plenum Press, New York and London, 1991.

[4] H.R. Krishna-murthy, K.G. Wilson, J.W. Wilson. Renormalization-Group Approach to the Anderson Model Of Dilute Magnetic Alloys. I.Static Properties for the Simetric Case. Phys. Rev.B. v.21,n.3,p.1003, 1980.

[5] Yoshida M.; Whitaker M.A.; Oliveira L.N. Renormalization-Group Calculation of Excitation Properties for Impurity Model. Phys. Rev. B v.41,n.13, p.9403,1990.

[6] Oliveira W.C.; Oliveira L.N. Generalized Numerical Renormalization Group Method to Calculate the Thermodinamical Properties of Impurities in Metals. Phys. Rev. B v.49, n.17, p. 11986, 1993.

[7] Anderson P.W. Localized Magnetic States in Metals. Phys. Rev. v.124, n.1, p.41, 1961

[8] Schiller A.; Hershfield S. Theory of Scannig Tunneling Spectroscopy of a Magnetic Adatom on a Metallic Surface. Phys. Rev. B v.61, n.13, p.9036, 2000 
